\title{
Cochlostoma revised: the subgenus Clessiniella Zallot et al., 2015 (Caenogastropoda, Cochlostomatidae)
}

\author{
Enrico ZALLOT ${ }^{\circledR 1, *}$, Willy DE MATTIA ${ }^{2}$, Zoltán FEHÉR ${ }^{3} \&$ Edmund GITTENBERGER ${ }^{4}$ \\ ${ }^{1}$ Haagweg 29, 2681PA Monster, the Netherlands. \\ ${ }^{2}$ Central Research Laboratories of Natural History Museum Vienna, Burgring 7, 1010 Vienna, Austria. \\ ${ }^{3}$ Hungarian Natural History Museum, Baross 13, 1088 Budapest, Hungary. \\ ${ }^{4}$ Naturalis Biodiversity Center, P.O. Box 9517, NL-2300RA Leiden, the Netherlands. \\ ${ }^{*}$ Corresponding author: ezallot@gmail.com \\ ${ }^{2}$ Email:wdemattia@gmail.com \\ ${ }^{3}$ Email: feher.zoltan@nhmus.hu \\ ${ }^{4}$ Email: egittenberger@yahoo.com

\footnotetext{
${ }^{1}$ urn:1sid:zoobank.org:author:365E17AD-6938-4364-A526-F1BDA2663E10

${ }^{2}$ urn:lsid:zoobank.org:author:C74A049D-9D7B-4A1F-878B-5BDFD104219F

${ }^{3}$ urn:lsid:zoobank.org:author:E801EC76-8B1E-450B-993E-BBBE57C00EA9

${ }^{4}$ urn:lsid:zoobank.org:author:D786C279-FC92-4D08-AF16-F79A9705E0AE
}

\begin{abstract}
Five species of the subgenus Clessiniella of Cochlostoma (Cochlostomatidae) are recognized, viz. Cochlostoma (Clessiniella) villae (Strobel, 1851), Cochlostoma (Clessiniella) tergestinum (Westerlund, 1878), Cochlostoma (Clessiniella) waldemari (A.J. Wagner, 1897), Cochlostoma (Clessiniella) anomphale Boeckel, 1939 and Cochlostoma (Clessiniella) stelucarum sp. nov. The shells and the genitalia are described and the distributional data are summarized for all the species.
\end{abstract}

Keywords. Taxonomy, morphology, Eastern Alps.

Zallot E., De Mattia W., Fehér Z. \& Gittenberger E. 2021. Cochlostoma revised: the subgenus Clessiniella Zallot et al., 2015 (Caenogastropoda, Cochlostomatidae). European Journal of Taxonomy 762: 49-95.

https://doi.org/10.5852/ejt.2021.762.1453

\section{Introduction}

The genus Cochlostoma Jan, 1830 includes predominantly rock-dwelling species distributed along the mountain ranges surrounding the Mediterranean Sea. Until recently, the taxonomy of the genus was based only on shell morphology. The lack of clear species-specific shell characters, however, makes it extremely difficult in many cases to assign a population to a certain species. In our previous papers (Zallot et al. 2015, 2018) we proved that the structure of the genitalia, together with the analyses of some DNA markers, may facilitate identifications while enabling a sound phylogenetic classification. The results of this approach imply a revision of the shell-based taxonomy, which is still used in the most recent European checklist (Welter-Schultes 2012). Whilst revising each of the subgenera of 
Cochlostoma, we herewith analyze the subgenus Clessiniella Zallot, Groenenberg, De Mattia, Fehér \& Gittenberger, 2015, which includes a group of phylogenetically closely related species inhabiting the eastern Alps and are characterized by a diagnostic structure of the female genitalia.

\section{Material and methods}

The sampling localities, mapped in Fig. 1, are also indicated for each species in the taxonomic section of this paper. An approximate position is used for the pre-GPS samples. The availability of female and male specimens from the different sampling localities was extremely various (dry or alcohol preserved, number of male and female specimens) and therefore the level of analysis of each sample was consequently different, as reported in Table 1. The methods for depicting the shells, obtaining measurements and dissecting genitalia have been discussed previously in Zallot (2002: 94-97) and Zallot et al. (2015: 64-67; 2018: 2-8). Here, we provide a description of the characters of the shell and of the genitalia that are used for classification.

\section{Abbreviations}

\section{Shell characters}

Ap. incl. = inclination of the apertural edge in lateral view in respect to the main axis (no. 13 in Fig. 2)

$\mathrm{H} 1^{\text {st }} 4^{\text {th }}=$ ratio between the height of the $1^{\text {st }}$ and that of the $4^{\text {th }}$ whorl. It is an index of the slenderness of the shell

$\mathrm{Ha} / \mathrm{Ht}=$ ratio between the height of the aperture measured along the main axis and the total height

$\mathrm{Ht}=$ total height: measured along the columella (no. 1 in Fig. 2)

$\mathrm{Ht} / \mathrm{W} \quad=$ ratio between the total height and the width of the basal whorl

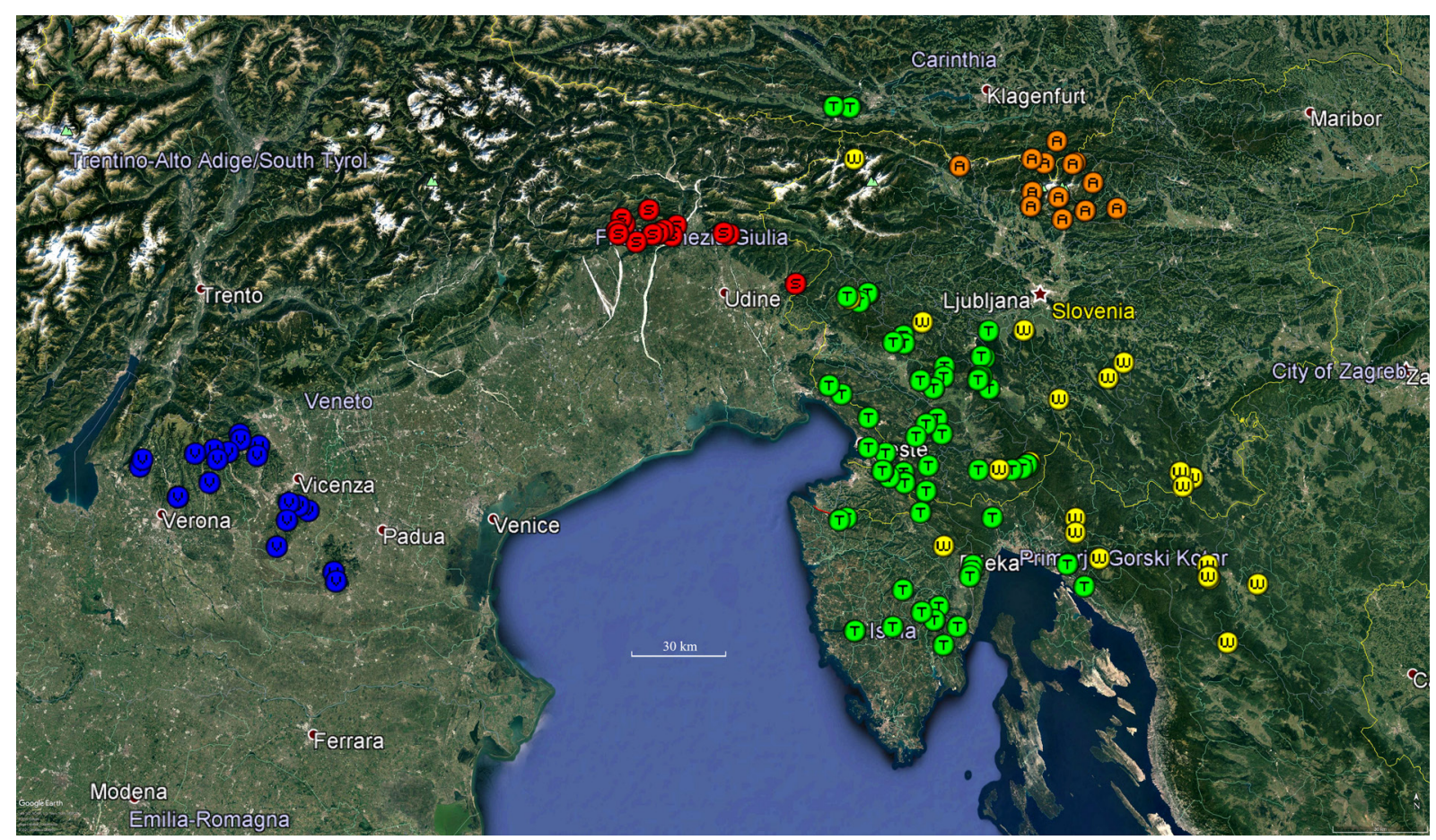

Fig. 1. Map of the sampling localities. Blue dots = Cochlostoma (Clessiniella) villae (Strobel, 1851); red dots $=C o .(C l$.$) stelucarum sp. nov.; green dots =C o .(C l$.$) tergestinum (Westerlund, 1878); yellow$ dots $=C o .(C l$.$) waldemari (A.J. Wagner, 1897); orange dots =C o .(C l$.$) anomphale Boeckel, 1939$. 


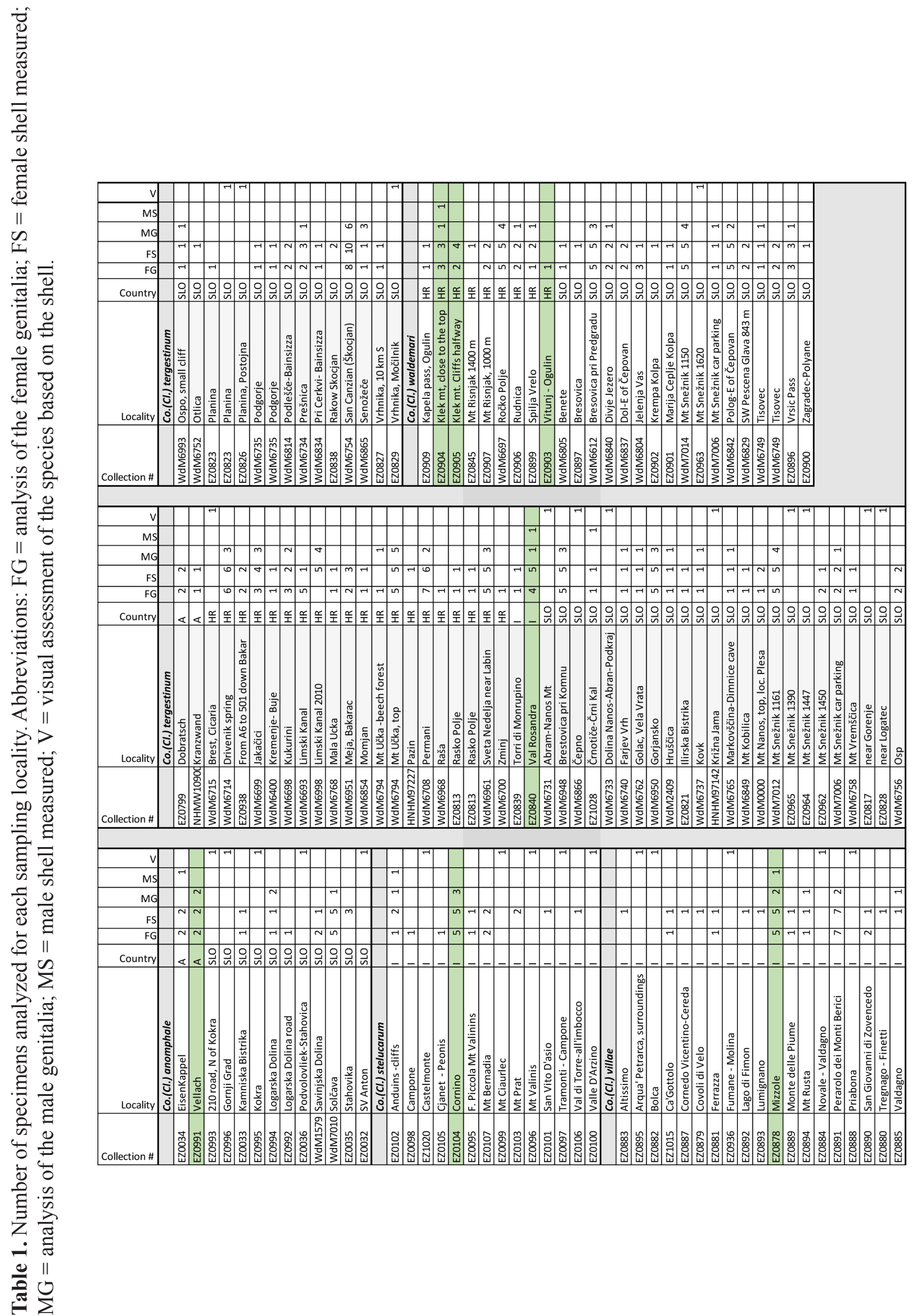


LipW = the apertural lip, on the columellar side, enlarges and flattens (no. 11 in Fig. 2). LipW is the ratio between the projection in frontal view of the maximum width of the lip on the columellar side and the maximum width of the aperture

LobeH $\quad=$ projection in frontal view of the height of the external lobe (no. 5 in Fig. 2)

Prt $=$ number of whorls of the protoconch. The embryonic shell is recognizable by a change of ribbing and often of the color (Fig. 3)

$\operatorname{Prt} / 4^{\text {th }}=$ ratio between the width of the basal whorl of the protoconch and the width of the shell measured along the $4^{\text {th }}$ suture. This ratio summarizes the relative size of the protoconch in respect to the whole shell

Ribs $/ \mathrm{mm}^{1^{\text {st }}}=$ number of ribs per $\mathrm{mm}$ on the $1^{\text {st }}$ whorl. The number of ribs per mm is calculated by measuring the distance between the first and the last of the most central five ribs close to the upper suture of the whorl (no. 15 in Fig. 2)

Ribs $/ \mathrm{mm} 4^{\text {th }}=$ number of ribs per $\mathrm{mm}$ on the $4^{\text {th }}$ whorl. The number of ribs per $\mathrm{mm}$ is calculated by measuring the distance between the first and the last of the most central five ribs of a whorl, in frontal view, close to the basal suture of the whorl (no. 16 in Fig. 2)
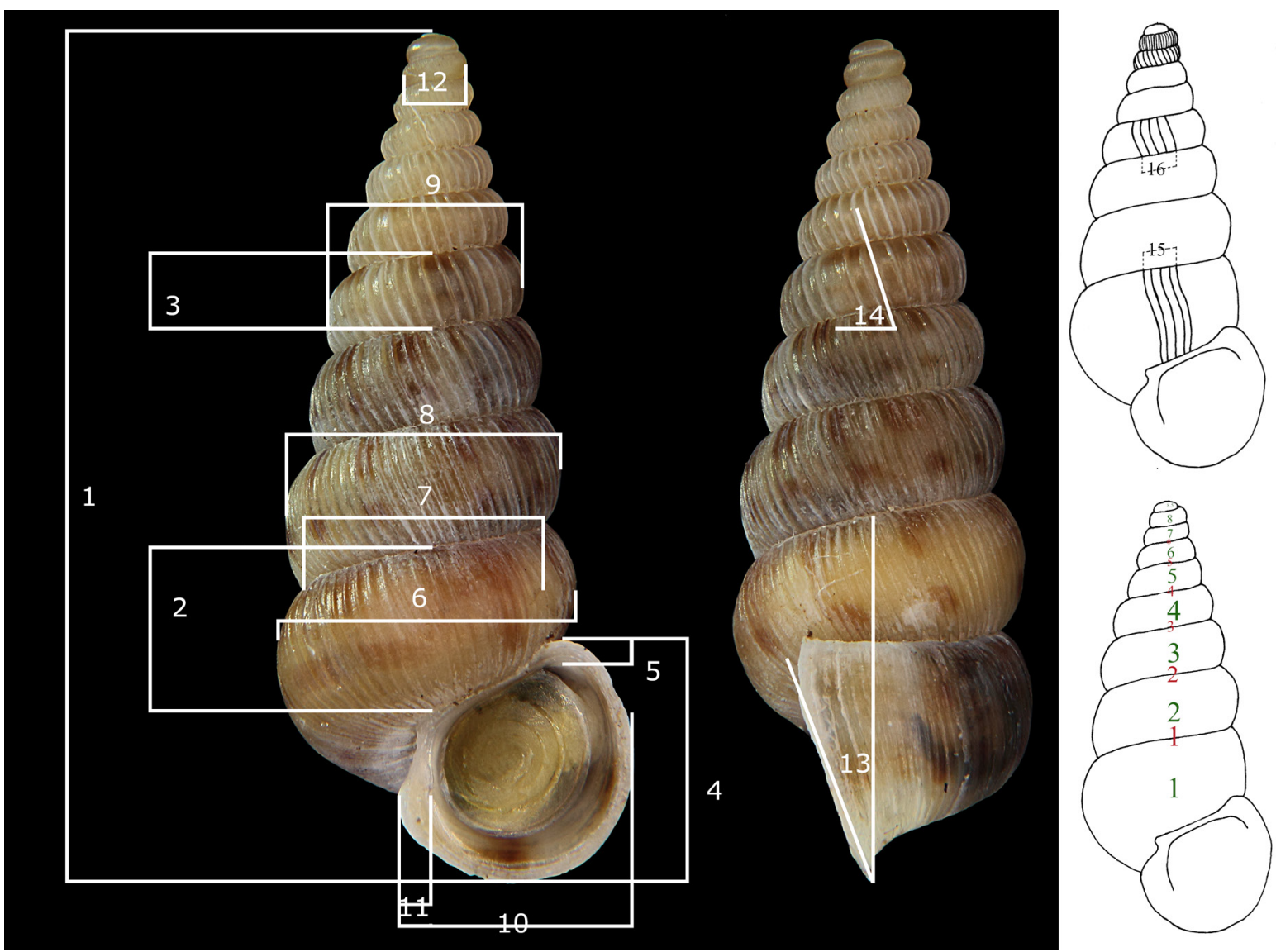

Fig. 2. Measurements taken on the shell of Clessiniella Zallot et al. 2015. $1=$ height (H); $2=$ height of the $1^{\text {st }}$ whorl; $3=$ height of the $4^{\text {th }}$ whorl; $4=$ height of the aperture $(\mathrm{Ha}) ; 5=$ height of the external lobe; 6 = width of the $1^{\text {st }}$ whorl $(\mathrm{W}) ; 7=$ width of the $1^{\text {st }}$ suture; $8=$ width of the $2^{\text {nd }}$ whorl; $9=$ width of the $4^{\text {th }}$ whorl; $10=$ width of the aperture $(\mathrm{Wa}) ; 11=$ maximum width of the lip on the columellar side; 12 = width of the basal whorl of the protoconch; $13=$ inclination of the aperture; $14=$ inclination of the ribs; $15=$ distance between the 5 central ribs of the body whorl; $16=$ distance between the 5 central ribs of the $4^{\text {th }}$ whorl. The figure in the lower right corner shows how the whorls (green) and sutures (red) are counted. 
Ribs incl. $\quad=$ inclination of the ribs in respect to the plane orthogonal to the columellar axis (no. 14 in Fig. 2)

Smooth Prt = number of protoconch whorls, starting from the tip point that are smooth at $40 \times$ magnification (no. 1 in Fig. 3)

$\mathrm{W}=$ width: measured at right angles to the columella (no. 6 in Fig. 2)

$\mathrm{W} 1^{\text {st }} / 4^{\text {th }}=$ ratio between the width of the $1^{\text {st }}$ and that of the $4^{\text {th }}$ whorl. It is an index of the slenderness of the shell

$\mathrm{W}$ round $\quad=$ ratio between the maximum width of the $2^{\text {nd }}$ whorl and the width of the shell measured at the $1^{\text {st }}$ suture. This measure indicates the convexity of the whorl(s), a feature frequently mentioned in the description of the taxa within Cochlostoma

Whorls $\quad=$ number of whorls: counted with the shell in front view. The whorls and the sutures are counted bottom-up, so that the basal whorl is the $1^{\text {st }}$ one and the suture above is the $1^{\text {st }}$ suture as shown in Fig. 2.

\section{Nominal or ordinal data}

External lobe = the body whorl, approaching the aperture, widens in different way and, as consequence, it changes the shape of the body whorl close to the aperture in external view (Fig. 4A)

Ribs near ap. = approaching the aperture, the ribs often become weaker in comparison to their strength on the front side of the body whorl. $\mathrm{W}=$ really weak ribs or smooth; I =

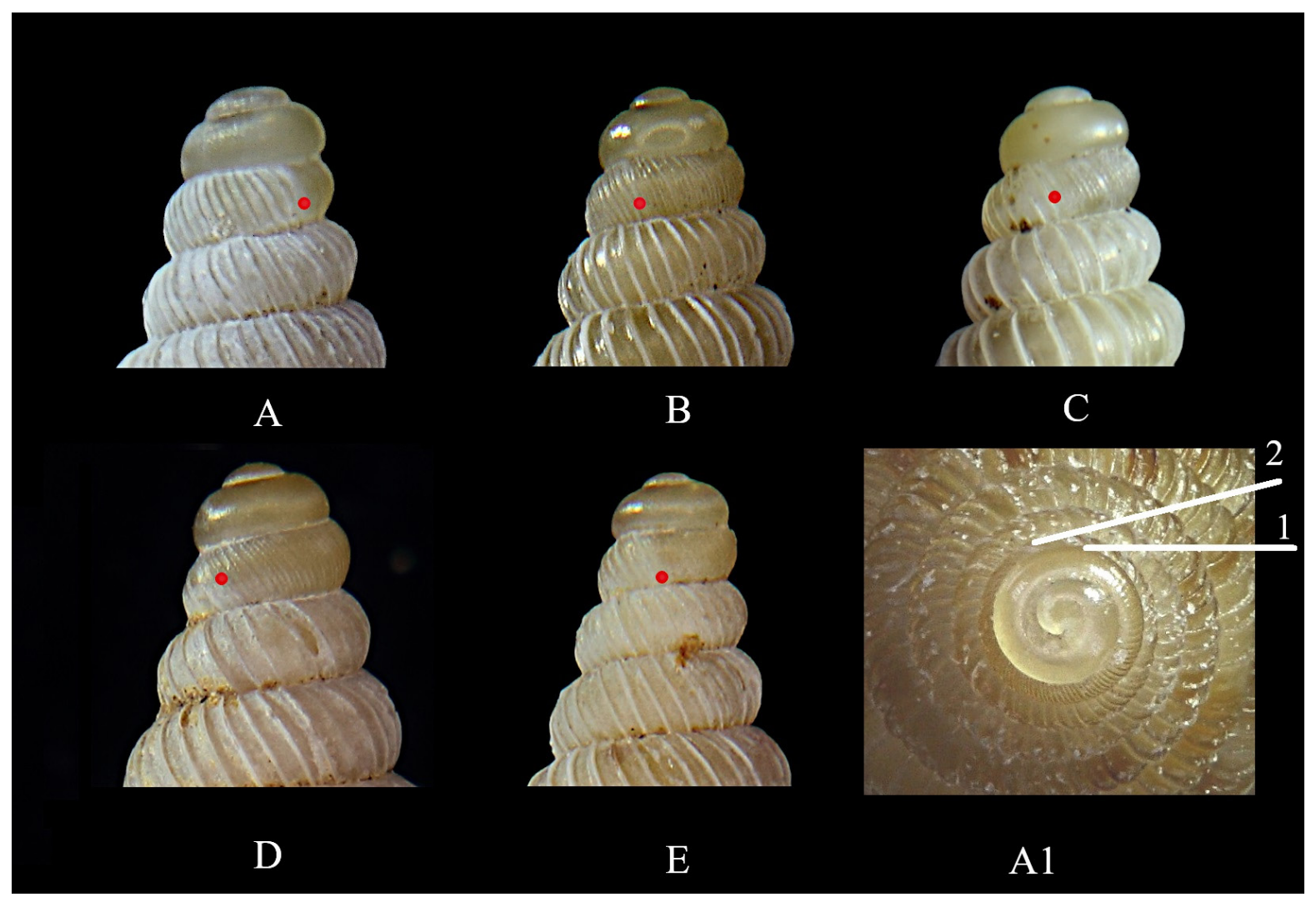

Fig. 3. Examples of protoconchs: the red dots mark the end of the protoconchs. A. Cochlostoma (Clessiniella) anomphale Boeckel, 1939 (EZ0034). A1. Co. (Cl.) anomphale (EZ0034): $1=$ end of the first smooth part of the protoconch; $2=$ end of the protoconch. B. Co. $(\mathrm{Cl}$.) tergestinum (Westerlund, 1878) (EZ0799). C. Co. (Cl.) villae (Strobel, 1851) (EZ0885). D. Co. (Cl.) waldemari (A.J. Wagner, 1897) (WdM6697). E. Co. (Cl.) stelucarum sp. nov. (EZ0104). 

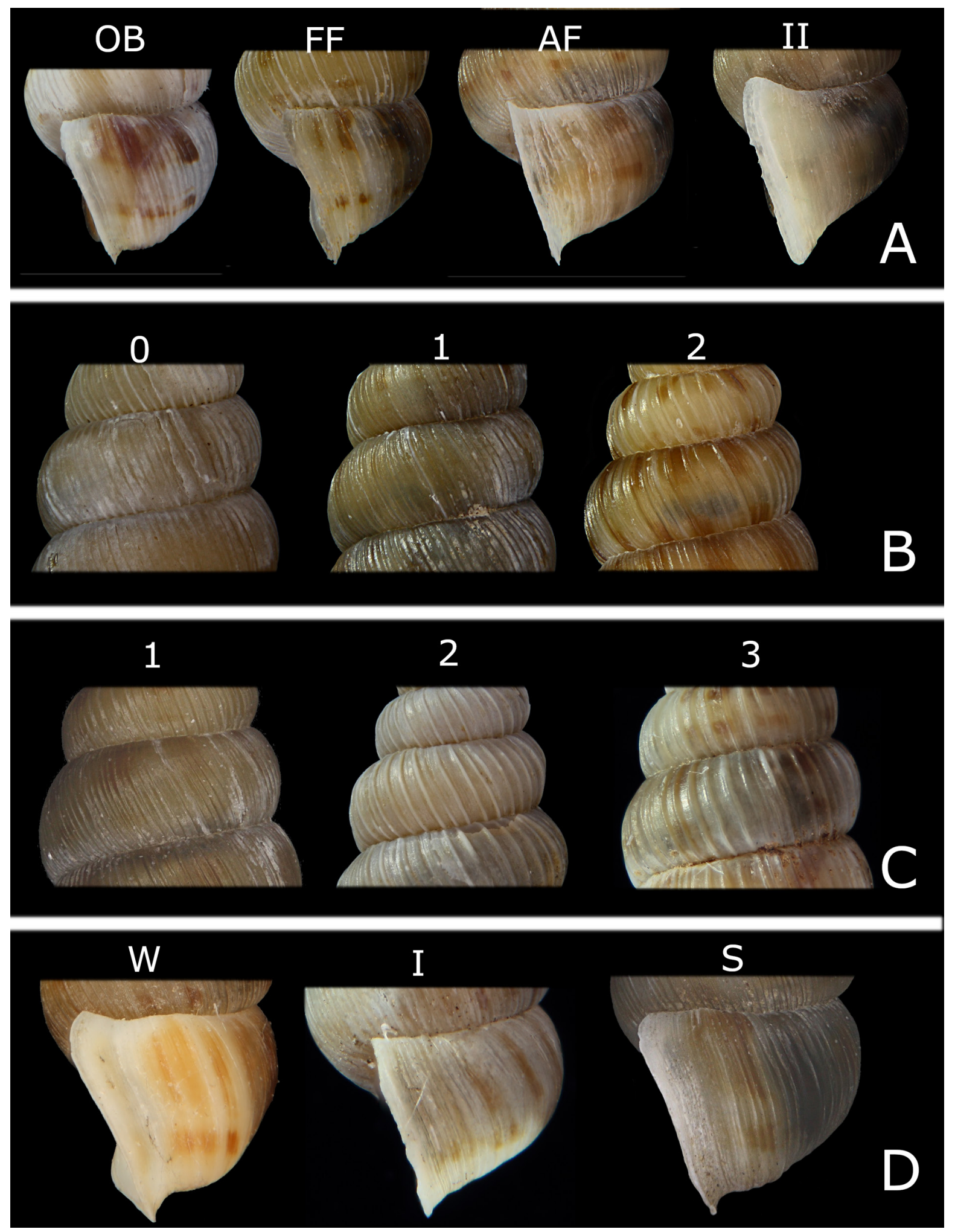

Fig. 4. Features of the shell of Clessiniella Zallot et al., 2015. A. Shape of the body whorl approaching the aperture. B. Strength of the spots on the shell $(0=$ spotless shell to $2=$ really evident spots $)$. C. Strength of the ribs $(1=$ weak ribs to 3 = really prominent ribs $)$. D. Strength of the ribs on the external side of the body whorl approaching the aperture $(\mathrm{W}=$ smooth shell; $\mathrm{I}=$ intermediate; $\mathrm{S}=$ prominent ribs). 
less strong than on the front side of the body whorl; $\mathrm{S}=$ as strong as in the front side (Fig. 4D)

Rib prominence $\quad=$ from $0=$ ribless shell to $3=$ really strong/prominent ribs (Fig. $4 \mathrm{C}$ )

Spots noticeability $=$ from $0=$ spotless shell to $2=$ really noticeable spots (Figs $4 \mathrm{~B}, 12$ ). The shell spots are areas of the shell that are darker than the background. They are usually reddish/brown.

Female genitalia (Figs 5-6)

Club-shaped seminal receptacle: seminal receptacle with narrow basis, gradually enlarging towards the apex where it reaches the maximum width: $\mathrm{SS}=$ short, club-shaped seminal receptacle; $\mathrm{SM}=$ clubshaped seminal receptacle of average length; $\mathrm{SL}=$ long, club-shaped seminal receptacle.

Oval-shaped seminal receptacle: seminal receptacle with recognizable basis and apex, with maximum width close to the midline; $\mathrm{BS}=$ short, oval-shaped seminal receptacle; $\mathrm{BM}=$ oval-shaped seminal receptacle of average length; $\mathrm{BL}=$ long, oval-shaped seminal receptacle.

Loops set up: the visceral oviduct, between the final loop and the seminal receptacle, is more or less tortuous and the resulting loops are more or less clustered together (Fig. 6B): $\mathrm{S}=$ superficial loops, quite distinct from one another; $\mathrm{O}=$ loops overlapping; $\mathrm{C}=$ loops tightly clustered.

Loops main position: the loops formed by the visceral oviduct can be differently arranged in relation to the apex of the seminal receptacle (Fig. 6C): $\mathrm{O}=$ loops positioned over (= dorsally) the apex of the seminal receptacle; $\mathrm{DH}=$ loops positioned close to the apex, on the visceral side; $\mathrm{DM}=$ loops positioned roughly halfway between the apex and the final loop.

CBC position: the pedunculus of the bursa copulatrix can connect to it in different relative positions: from $0=$ apically to $3=$ ventrally to $4=$ distally (Fig. $6 \mathrm{D})$.

SRwidth: Fig. 6E.

SR apex position: position of the apex of the seminal receptacle relative to the body: from 0 to $0.5=$ ventral side of the body; from 0.6 to $1=$ dorsal side of the body (Fig. $6 \mathrm{G}$ )

SR curvature: the seminal receptacle can have a portion of its apical part (the one connected to the visceral oviduct) curved downwards (apex towards the ventral side of the body) (Fig. 6F): from $0=$ apex straight, not curved to $0.5=$ half seminal receptacle curved to $1=$ inverted seminal receptacle (connection with the distal oviduct on the dorsal side of the body and connection with the visceral oviduct on the ventral side of the body).

JUG position: the junction of the uterus gland with the copulatory channel can be more or less far from the connection between the pedunculus of the bursa and the distal oviduct: from $0=$ really close to $3=$ really far (Fig. 6H).

Loops n.: the visceral oviduct can have a different number of loops. A rough count of them gives a measure of the complexity of the loops path (and of the length of the visceral oviduct in between the final loop and the apex of the seminal receptacle).

\section{DNA sequence analysis}

For DNA extraction from small pieces of tissue of ethanol preserved specimens we used the DNeasy Blood \& Tissue Kit (Qiagen) following the manufacturer's protocol. A $309 \mathrm{bp}$ long gene fragment of the nuclear Histone $\mathrm{H} 3$ gene was amplified by polymerase chain reaction (PCR) using the following 
primers: H3ColF (Colgan et al. 2000) and H3PulR (Uit de Weerd \& Gittenberger 2013). For PCR, TopTaq DNA Polymerase (Qiagen) was used. The PCR profile started with an initial denaturation for $3 \mathrm{~min}$ at $94^{\circ} \mathrm{C}$, followed by 40 cycles of $30 \mathrm{~s}$ at $94^{\circ} \mathrm{C}$, annealing for $30 \mathrm{~s}$ at $57^{\circ} \mathrm{C}$ and elongation for $60 \mathrm{~s}$ at $72^{\circ} \mathrm{C}$, terminated by a final elongation phase of $10 \mathrm{~min}$ at $72^{\circ} \mathrm{C}$. Sequencing (both directions) was performed by LGC Genomics (Berlin, Germany) using the PCR primers.

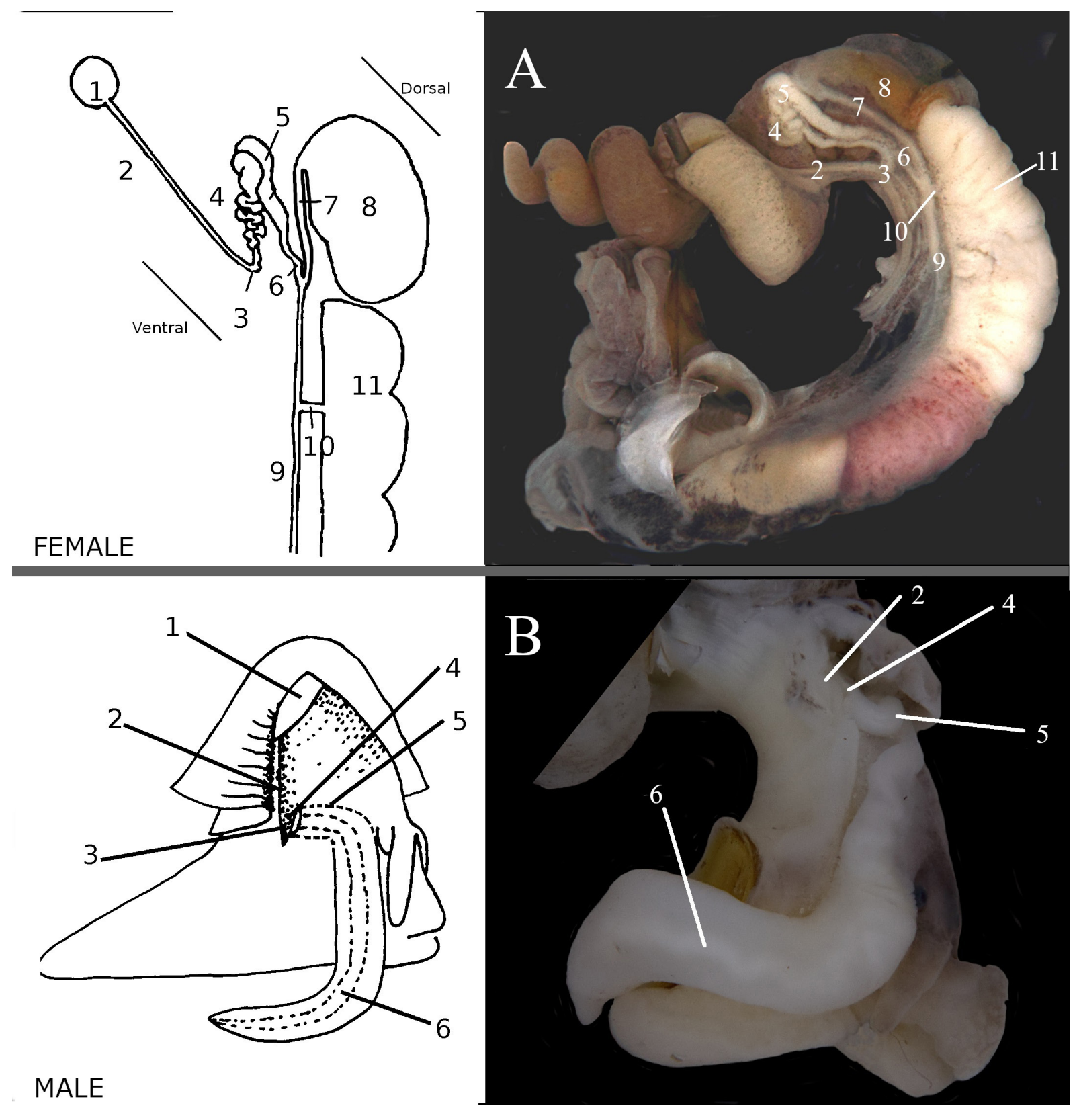

Fig. 5. Genitalia in Clessiniella Zallot et al., 2015. A. Female genitalia (Cochlostoma (Clessiniella) tergestinum (Westerlund, 1878) (WdM6998)). 1 = ovary; 2 = visceral oviduct; 3 = proximal loop; $4=$ loops of the visceral oviduct; $5=$ seminal receptacle; $6=$ distal oviduct; $7=$ pedunculus of the bursa copulatrix; $8=$ bursa copulatrix; $9=$ copulatory duct; $10=$ channel of the uterine gland; $11=$ uterine gland. B. Male genitalia $(\mathrm{Co}$. $(\mathrm{Cl}$.) tergestinum (WdM7012)). $1=$ anus; $2=$ fold delimiting the sperm groove; $3=$ sperm pocket; $4=$ penial funnel; $5=$ body spermiduct; $6=$ penis with the internal spermiduct. 


\section{Institutional abbreviations}

$\begin{array}{ll}\text { EZ } & \text { private collection of Enrico Zallot } \\ \text { MIZPAS } & =\text { Muzeum i Instytut Zoologii PAN, Warsaw, Poland } \\ \text { NHMW } & \text { Naturhistorisches Museum, Vienna, Austria } \\ \text { PU } & =\text { Museo di Storia Naturale dell'Universita' di Parma, Italy } \\ \text { RMNH } & =\text { Naturalis Biodiversity Center, Leiden, the Netherlands } \\ \text { SMF } & =\text { Naturmuseum Senckenberg, Frankfurt am Main, Germany } \\ \text { WDM } & =\text { private collection of Willy De Mattia }\end{array}$

\section{Results}

\section{Morphological characters}

Five species of Cochlostoma, one of which is new to science and here described, show the female genital characters of the subgenus Clessiniella as described by Zallot et al. (2015: 82). In Table 2 we present the measurements of the shell characters of the types and the average, minimum, maximum and standard

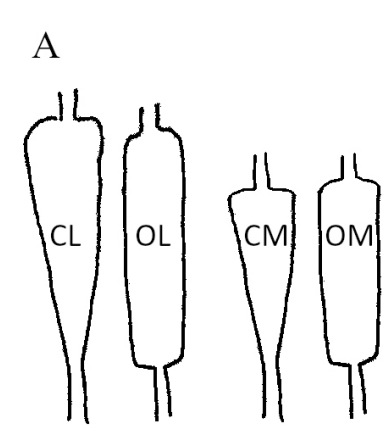

Seminal receptacle shape

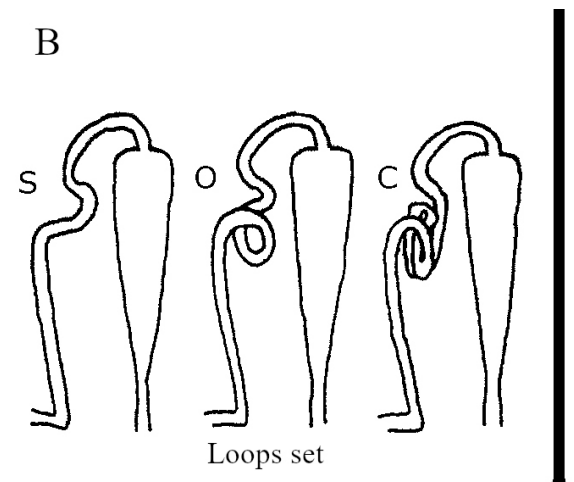

$\mathrm{C}$
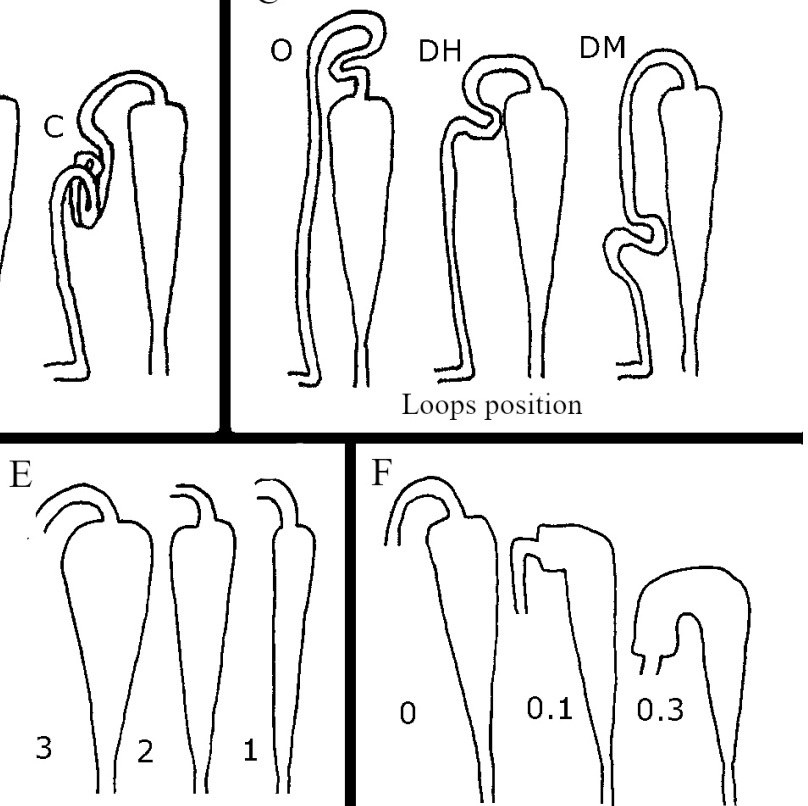

Width of the seminal recep.

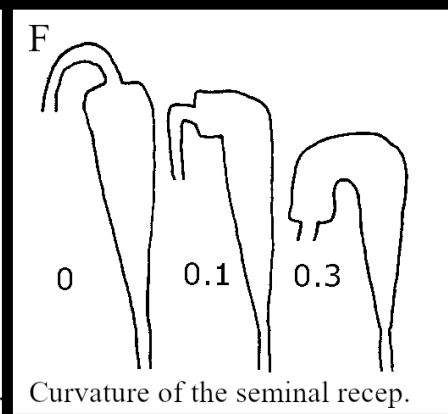

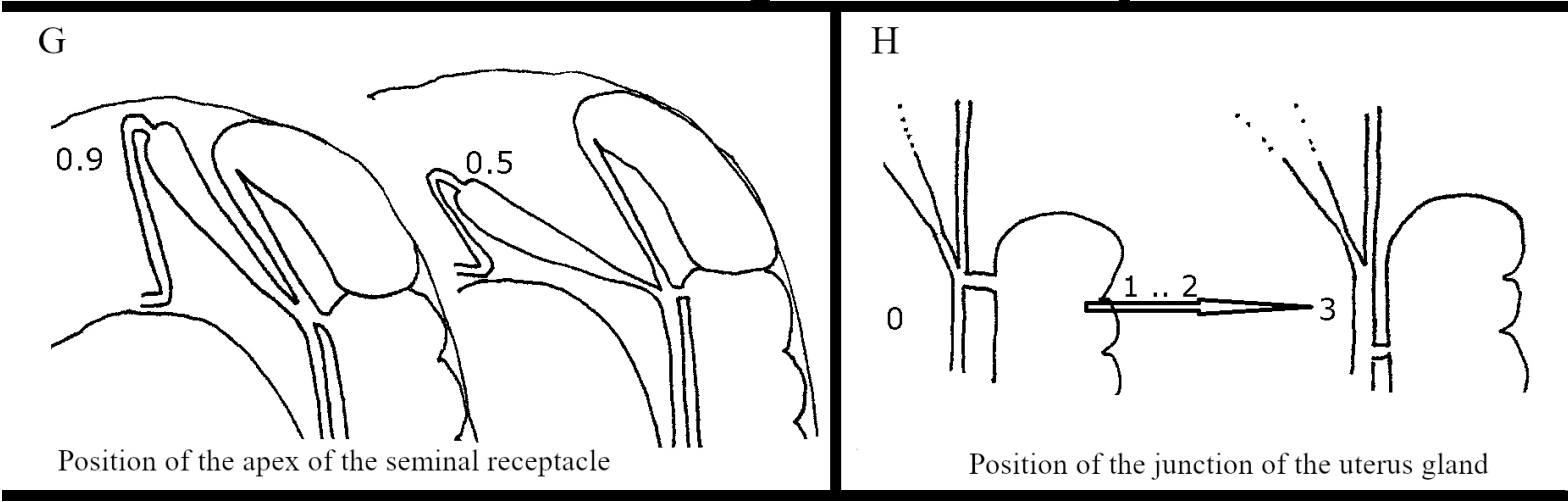

Fig 6. Female genital features. A. SR shape. B. Loops set up. C. Loops main position. D. CBC position. E. SR width. F. SR curvature. G. SR apex position. H. JUG position. 
Table 2. Shell measurements. Abbreviation: $\mathrm{N}=$ number of female specimens analyzed.

\begin{tabular}{|c|c|c|c|c|c|c|c|c|c|c|c|c|c|c|c|c|c|c|}
\hline & & 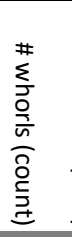 & $\begin{array}{l}\frac{I}{3} \\
\underline{3} \\
\end{array}$ & $\frac{\Sigma}{3}$ & $\sum_{\Sigma}^{I}$ & $\stackrel{\text { I }}{\text { I }}$ & $\begin{array}{l}\text { I } \\
\stackrel{5}{y} \\
\stackrel{5}{f} \\
\text { f }\end{array}$ & 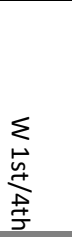 & 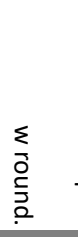 & $\frac{\text { 둥 }}{\sum}$ & $\begin{array}{l}5 \\
\frac{0}{0} \\
\mathbb{D} \\
I\end{array}$ & 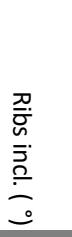 & 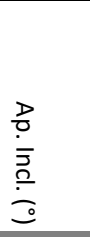 & 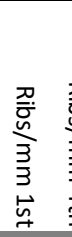 & 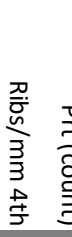 & 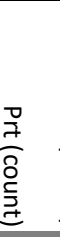 & 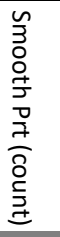 & 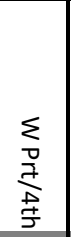 \\
\hline \multirow{5}{*}{ Clessiniella } & $\mathrm{N}$ & 214 & 215 & 215 & 215 & 215 & 215 & 215 & 215 & 215 & 215 & 215 & 198 & 2042 & 215 & 141 & 135 & \begin{tabular}{|l|l|}
5 & 196 \\
\end{tabular} \\
\hline & Average & 8.4 & 7.9 & 3.0 & 2.6 & \begin{tabular}{|l|}
0.22 \\
\end{tabular} & 2.6 & 1.9 & 1.15 & 0.14 & \begin{tabular}{|l|}
0.12 \\
\end{tabular} & 66.5 & 15.7 & 8 & \begin{tabular}{l|l}
7 \\
\end{tabular} & 2.2 & 1.5 & 0.61 \\
\hline & Min & 7.2 & 6.1 & 2.5 & 2.3 & 0.18 & 2.0 & 1.6 & 1.02 & 0.06 & 0.03 & 59.3 & 5.4 & 4 & 4 & 1.8 & 1.1 & 0.42 \\
\hline & Max & 10.3 & 10.8 & 3.8 & 3.0 & \begin{tabular}{|l|}
0.27 \\
\end{tabular} & 3.4 & 2.2 & 1.20 & 0.21 & 0.23 & 76.5 & 26.5 & 17 & 14 & 2.5 & 1.8 & 0.81 \\
\hline & StdDev & 0.6 & 0.7 & 0.2 & 0.1 & \begin{tabular}{|l|}
0.02 \\
\end{tabular} & \begin{tabular}{|l|}
0.2 \\
\end{tabular} & 0.1 & \begin{tabular}{|l|}
0.02 \\
\end{tabular} & 0.03 & 0.03 & 3.3 & 3.9 & 2 & 2 & 0.1 & 0.1 & 0.08 \\
\hline \multirow[t]{8}{*}{ C. (C.) anomphale } & paratype 19 & 7.5 & 6.2 & 2.56 & 2.43 & 0.23 & 2.75 & 2.01 & \begin{tabular}{|l|}
0.13 \\
\end{tabular} & 0.18 & 0.11 & \begin{tabular}{|l|}
65.4 \\
\end{tabular} & 18.6 & 10 & \begin{tabular}{l|l}
10 & 0 \\
\end{tabular} & 0.67 & & 0.69 \\
\hline & paratype 29 & 7.2 & 6.2 & 2.63 & 2.35 & 0.22 & 2.98 & 2.09 & 0.15 & 0.14 & 0.10 & 64.8 & 15.7 & 11 & \begin{tabular}{l|l}
99 & 0 \\
\end{tabular} & 0.71 & & 0.73 \\
\hline & & & & & & & & & & & & & & & & & & \\
\hline & $\mathrm{N}$ & 15 & 15 & 15 & 15 & 15 & 15 & 15 & 15 & 15 & 15 & 15 & 14 & 15 & 15 & 10 & 10 & 13 \\
\hline & Average & 8.4 & 7.8 & 3.0 & 2.6 & \begin{tabular}{|l|}
0.21 \\
\end{tabular} & 2.6 & 1.9 & 1.15 & 0.13 & \begin{tabular}{|l|}
0.07 \\
\end{tabular} & 67.3 & 16.4 & 11 & 9 & 2.2 & 1.4 & 0.56 \\
\hline & Min & 8.0 & 6.9 & 2.8 & 2.5 & 0.19 & 2.2 & 1.7 & 1.11 & 0.10 & 0.03 & 64.2 & 9.3 & 7 & 7 & 2.0 & 1.3 & 0.49 \\
\hline & Max & 9.1 & 8.5 & 3.2 & 2.7 & 0.22 & 3.0 & 2.1 & 1.17 & 0.17 & 0.11 & 69.7 & 20.3 & 17 & 14 & 2.3 & 1.6 & 0.65 \\
\hline & StdDev & 0.4 & 0.4 & 0.1 & 0.1 & \begin{tabular}{|l|}
0.01 \\
\end{tabular} & \begin{tabular}{|l|}
0.2 \\
\end{tabular} & 0.1 & \begin{tabular}{|l|}
0.02 \\
\end{tabular} & \begin{tabular}{|l|}
0.02 \\
\end{tabular} & \begin{tabular}{|l|}
0.02 \\
\end{tabular} & 2.1 & 3.5 & 3 & 2 & 0.1 & 0.1 & 0.05 \\
\hline \multirow{8}{*}{ C. (C.) stelucarum } & & & & & & & & & & & & & & & & & & \\
\hline & \begin{tabular}{|l} 
holotype + \\
paratype $\%$
\end{tabular} & \begin{tabular}{|l|}
10.0 \\
10.3
\end{tabular} & $\begin{array}{l}8.0 \\
8.7\end{array}$ & \begin{tabular}{|l|}
3.20 \\
3.09 \\
\end{tabular} & 2.81 & \begin{tabular}{|l|}
0.19 \\
\end{tabular} & 2.47 & $\mid 1.67$ & \begin{tabular}{|l|}
0.17 \\
\end{tabular} & \begin{tabular}{|l|}
0.14 \\
\end{tabular} & \begin{tabular}{|l|}
0.07 \\
\end{tabular} & 69.3 & 17.7 & 10 & $6 \mid 0$ & 0.64 & & 0.43 \\
\hline & paratype $\sigma^{n}$ & 9.1 & 7.0 & 3.00 & & & & & & & & & & & & & & \\
\hline & $\mathrm{N}$ & 14 & 14 & 14 & 14 & 14 & 14 & 14 & 14 & 14 & 14 & 14 & 11 & 14 & 14 & 8 & 7 & 14 \\
\hline & Average & 9.6 & 8.4 & 3.1 & 2.7 & 0.20 & 2.3 & 1.8 & \begin{tabular}{|l|}
1.14 \\
\end{tabular} & 0.12 & \begin{tabular}{|l|}
0.08 \\
\end{tabular} & \begin{tabular}{|l|}
70.0 \\
\end{tabular} & 18.4 & 8 & 7 & 2.2 & 1.5 & 0.48 \\
\hline & Min & 8.4 & 7.4 & 2.9 & 2.5 & 0.18 & \begin{tabular}{|l|}
2.0 \\
\end{tabular} & 1.6 & \begin{tabular}{|l|}
1.11 \\
\end{tabular} & 0.08 & \begin{tabular}{|l|}
0.04 \\
\end{tabular} & 66.5 & 15.2 & 5 & 5 & 2.1 & 1.3 & 0.42 \\
\hline & Max & 10.3 & 9.5 & 3.3 & 2.8 & \begin{tabular}{|l|}
0.22 \\
\end{tabular} & 2.6 & 1.9 & \begin{tabular}{|l|}
1.17 \\
\end{tabular} & 0.14 & \begin{tabular}{|l|}
0.12 \\
\end{tabular} & 75.7 & 20.2 & 11 & 8 & 2.3 & 1.8 & 0.58 \\
\hline & StdDev & 0.6 & 0.8 & 0.2 & 0.1 & 0.01 & \begin{tabular}{|l|}
0.2 \\
\end{tabular} & 0.1 & \begin{tabular}{|l|l|}
0.02 \\
\end{tabular} & \begin{tabular}{|l|l|}
0.02 \\
\end{tabular} & \begin{tabular}{|l|}
0.02 \\
\end{tabular} & 2.2 & 1.7 & 2 & 1 & 0.1 & 0.2 & 0.06 \\
\hline \multirow[t]{8}{*}{ C. (C.) tergestinum } & syntype 9 & 9.1 & 7.8 & \begin{tabular}{|l|}
2.83 \\
\end{tabular} & 2.75 & \begin{tabular}{|l|}
0.22 \\
\end{tabular} & 2.70 & 1.88 & \begin{tabular}{|l|}
0.13 \\
\end{tabular} & \begin{tabular}{|l|}
0.16 \\
\end{tabular} & \begin{tabular}{|l|}
0.14 \\
\end{tabular} & \begin{tabular}{|l|}
68.0 \\
\end{tabular} & 13.9 & 6 & \begin{tabular}{l|l}
4 & 0 \\
\end{tabular} & 0.65 & & 0.54 \\
\hline & syntype $0^{\prime \prime}$ & 8.2 & 7.4 & 2.98 & 2.49 & 0.23 & 2.66 & 1.55 & 0.13 & 0.12 & \begin{tabular}{|l|}
0.12 \\
\end{tabular} & 66.8 & 12.6 & 6 & $5 \mid 0$ & 0.77 & & 0.52 \\
\hline & & & & & & & & & & & & & & & & & & \\
\hline & $\mathrm{N}$ & 108 & 108 & 108 & 108 & 108 & 108 & 108 & 108 & 108 & \begin{tabular}{|l|}
108 \\
\end{tabular} & 108 & 99 & 102 & 108 & 69 & $67 \mid$ & 93 \\
\hline & Average & 8.3 & 7.8 & 3.0 & 2.6 & \begin{tabular}{|l|}
0.22 \\
\end{tabular} & \begin{tabular}{|l|}
2.7 \\
\end{tabular} & 2.0 & 1.15 & 0.14 & \begin{tabular}{|l|}
0.12 \\
\end{tabular} & 65.5 & 16.9 & 8 & 7 & 2.2 & 1.5 & 0.63 \\
\hline & Min & 7.3 & 6.1 & 2.5 & 2.3 & \begin{tabular}{|l|}
0.18 \\
\end{tabular} & 2.3 & 1.7 & 1.02 & 0.06 & 0.05 & \begin{tabular}{|l|}
59.3 \\
\end{tabular} & 5.9 & 5 & 4 & 1.8 & \begin{tabular}{|l|}
1.1 \\
\end{tabular} & 0.51 \\
\hline & Max & 10.0 & 9.6 & 3.4 & 2.9 & 0.27 & 3.2 & 2.2 & 1.20 & 0.20 & 0.23 & 70.2 & 26.5 & 16 & 13 & 2.5 & 1.7 & 0.76 \\
\hline & StdDev & 0.4 & 0.7 & 0.2 & 0.1 & \begin{tabular}{|l|}
0.02 \\
\end{tabular} & 0.2 & 0.1 & \begin{tabular}{|l|}
0.02 \\
\end{tabular} & 0.03 & 0.04 & 2.3 & 3.4 & 2 & \begin{tabular}{|l|l|}
1 & \\
\end{tabular} & 0.1 & 0.1 & 0.06 \\
\hline \multirow[t]{6}{*}{ C. (C.) villae } & syntype $\$$ & 9.2 & 10.1 & 3.43 & 2.94 & \begin{tabular}{|l|}
0.19 \\
\end{tabular} & 1.8 & 1.62 & \begin{tabular}{|l|}
0.14 \\
\end{tabular} & \begin{tabular}{|l|} 
\\
\end{tabular} & \begin{tabular}{|l|}
0.09 \\
\end{tabular} & 75.6 & 18.18 & 5 & $4 \mid-$ & & & - \\
\hline & $\mathrm{N}$ & 23 & 24 & 24 & 24 & 24 & 24 & 24 & 24 & 24 & 24 & 24 & 22 & 23 & 24 & 16 & 15 & 24 \\
\hline & Average & 8.8 & 8.8 & 3.3 & 2.7 & 0.22 & 2.6 & 1.8 & 1.13 & 0.13 & 0.10 & 72.1 & 15.7 & 6 & 5 & 2.3 & 1.4 & 0.53 \\
\hline & Min & 8.2 & 7.1 & 2.9 & 2.5 & 0.20 & 2.2 & 1.7 & 1.09 & 0.08 & 0.06 & 64.6 & 6.5 & 4 & \begin{tabular}{l|l}
4 \\
\end{tabular} & 2.0 & 1.3 & 0.43 \\
\hline & Max & 9.4 & 10.8 & 3.8 & 3.0 & \begin{tabular}{|l|} 
\\
\end{tabular} & 3.2 & 2.0 & \begin{tabular}{|l|}
1.19 \\
\end{tabular} & \begin{tabular}{|l|} 
\\
\end{tabular} & 0.15 & 76.5 & 21.7 & 9 & 8 & 2.4 & 1.7 & 0.63 \\
\hline & StdDev & 0.4 & 0.9 & 0.3 & 0.1 & \begin{tabular}{|l|}
0.01 \\
\end{tabular} & 0.2 & 0.1 & \begin{tabular}{|l|} 
\\
\end{tabular} & 0.03 & \begin{tabular}{|l|} 
\\
\end{tabular} & 2.6 & 3.2 & 1 & 1 & 0.1 & 0.2 & 0.05 \\
\hline \multirow[t]{6}{*}{ C. (C.) waldemari } & syntype 9 & 7.5 & 7.3 & \begin{tabular}{|l|}
2.72 \\
\end{tabular} & 2.68 & 0.26 & 2.93 & 1.93 & \begin{tabular}{|l|}
0.15 \\
\end{tabular} & 0.16 & 0.15 & \begin{tabular}{|l|}
64.7 \\
\end{tabular} & 4.9 & 8 & \begin{tabular}{l|l}
11 & 0 \\
\end{tabular} & 0.72 & & 0.70 \\
\hline & $\mathrm{N}$ & 54 & 54 & 54 & 54 & 54 & 54 & 54 & 54 & 54 & 54 & 54 & 52 & 50 & \begin{tabular}{|l|}
54 \\
\end{tabular} & 38 & \begin{tabular}{|l|}
36 \\
\end{tabular} & 52 \\
\hline & Average & 8.0 & 7.8 & 3.1 & 2.5 & \begin{tabular}{|l|} 
\\
\end{tabular} & 2.7 & 2.0 & 1.14 & 0.15 & \begin{tabular}{|l|} 
\\
\end{tabular} & 65.0 & 12.9 & 9 & 9 & 2.2 & 1.4 & $\begin{array}{l} \\
\end{array}$ \\
\hline & Min & 7.2 & 7.0 & 2.8 & 2.4 & 0.21 & 2.3 & 1.7 & 1.10 & 0.10 & 0.06 & 60.0 & 5.4 & 5 & 5 & 2.0 & 1.1 & 0.55 \\
\hline & Max & 8.6 & 8.8 & 3.4 & 2.7 & 0.27 & 3.4 & 2.2 & 1.19 & 0.21 & 0.18 & 69.7 & 20.4 & 14 & \begin{tabular}{|l|l|}
14 \\
\end{tabular} & 2.5 & 1.7 & 0.81 \\
\hline & StdDev & 0.3 & 0.4 & 0.1 & 0.1 & \begin{tabular}{|l|} 
\\
\end{tabular} & \begin{tabular}{|l|}
0.2 \\
\end{tabular} & 0.1 & \begin{tabular}{|l|}
0.02 \\
\end{tabular} & \begin{tabular}{|l|}
0.03 \\
\end{tabular} & \begin{tabular}{|l|}
0.02 \\
\end{tabular} & 2.4 & 3.9 & 2 & 2 & 0.1 & 0.1 & 0.05 \\
\hline
\end{tabular}


Table 3. Percentage of the analyzed female specimens presenting the shell feature as explained in Fig. 3 within each species of Clessiniella Zallot et al., 2015. In bold the feature status as presented by the types. Abbreviation: $\mathrm{N}=$ number of analyzed specimens.

\begin{tabular}{|c|c|c|c|c|c|}
\hline 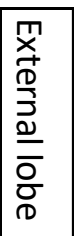 & $\begin{array}{l}\frac{1}{3} \\
\frac{0}{3} \\
\frac{0}{0} \\
\frac{0}{0} \\
\frac{0}{0}\end{array}$ & $\begin{array}{l}\frac{n}{0} \\
\frac{0}{2} \\
\frac{2}{5} \\
\frac{2}{5} \\
\frac{5}{3}\end{array}$ & 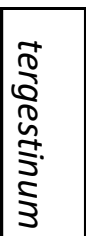 & 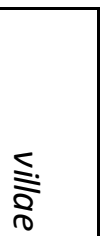 & 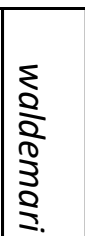 \\
\hline$N$ & 16 & 14 & 108 & 24 & 55 \\
\hline $\mathrm{FF}$ & & $43 \%$ & $25 \%$ & $83 \%$ & $2 \%$ \\
\hline$A F$ & & $57 \%$ & $15 \%$ & & $9 \%$ \\
\hline II & & & $60 \%$ & $13 \%$ & $89 \%$ \\
\hline OB & $100 \%$ & & & $4 \%$ & \\
\hline
\end{tabular}
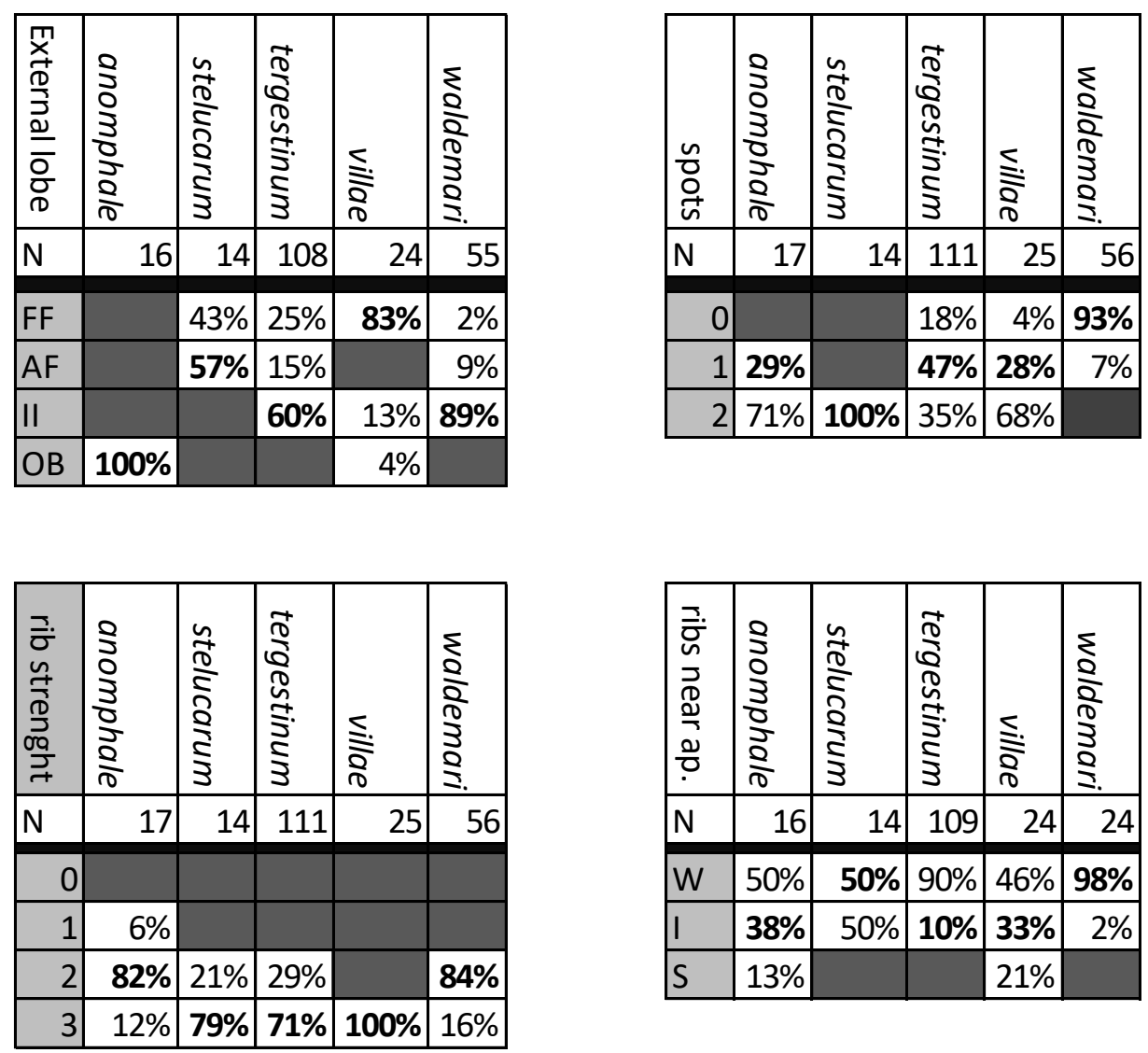

\begin{tabular}{|c|c|c|c|c|c|}
\hline 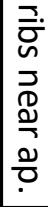 & $\begin{array}{l}\frac{0}{3} \\
\frac{0}{3} \\
\frac{3}{2} \\
\frac{0}{2} \\
\frac{0}{2}\end{array}$ & 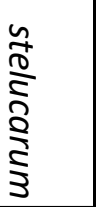 & 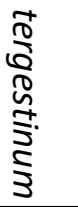 & $\underset{\frac{1}{2}}{\lessgtr}$ & 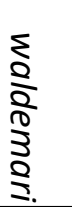 \\
\hline $\mathrm{N}$ & 16 & 14 & 109 & 24 & 24 \\
\hline W & $50 \%$ & $50 \%$ & $90 \%$ & $46 \%$ & $98 \%$ \\
\hline 1 & $38 \%$ & $50 \%$ & $10 \%$ & $33 \%$ & $2 \%$ \\
\hline$S$ & $13 \%$ & & & $21 \%$ & \\
\hline
\end{tabular}

deviation values of the characters at the subgenus and species level. In Table 3 we report of each species the features as explained in Fig. 3. A discriminant analysis based on the shell features is presented in Fig. 8. In Figs 9-11 the relations between some of the most relevant shell characters are graphically illustrated.

Female genitalia. The data regarding the female genital features are presented in Table 4 . 
Table 4. Number of female specimens presenting the genital features as explained in Fig. 5. Abbreviation: $\mathrm{N}=$ number of analyzed specimens.

\begin{tabular}{|c|c|c|c|c|c|c|c|c|c|c|c|c|c|c|c|c|c|c|c|c|c|}
\hline & 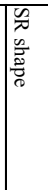 & & 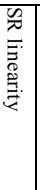 & 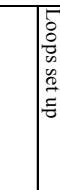 & & 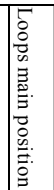 & & 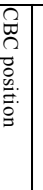 & & 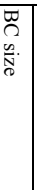 & & 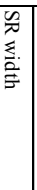 & & 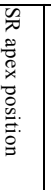 & & 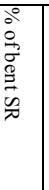 & & 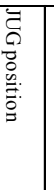 & & 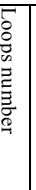 & \\
\hline \multirow[t]{7}{*}{ Clessiniella } & $\mathrm{N}$ & 196 & & 196 & 196 & & 195 & & 195 & & 189 & & 196 & & 185 & & 194 & & 171 & & 195 \\
\hline & SM & 19 & & \begin{tabular}{l|l}
190 & $\mathrm{~S}$ \\
\end{tabular} & & $\mathrm{O}$ & 6 & & 190 & 1 & 6 & 1 & 18 & $\leq 0.5$ & 6 & 0 & 51 & & 114 & & 13 \\
\hline & SL & 166 & $\mathrm{U}$ & 60 & & $2 \mathrm{DH}$ & 69 & 1 & 5 & 2 & 43 & 2 & 110 & 0.6 & 15 & 0.1 & 72 & 0.5 & 36 & 2 & 40 \\
\hline & BM & 1 & $\mathrm{~T}$ & $\mathrm{C}$ & & $\mathrm{DM}$ & 120 & 2 & & 3 & 114 & 3 & 68 & 0.7 & 45 & 0.2 & 48 & 1 & 21 & 3 & 57 \\
\hline & $\mathrm{BL}$ & 10 & & & & & & 3 & & 4 & 26 & & & 0.8 & 64 & 0.3 & 15 & 1.5 & & 4 & 51 \\
\hline & & & & & & & & 4 & & & & & & $\geq 0.9$ & 55 & $\geq 0.4$ & 8 & 2 & & & 28 \\
\hline & & & & & & & & & & & & & & & & & & $\geq 2.5$ & & $\geq 6$ & 6 \\
\hline \multirow[t]{7}{*}{ C.(C.) anomphale } & $\mathrm{N}$ & 13 & & 13 & 13 & & 13 & & 13 & & 13 & & 13 & & 12 & & 13 & & 12 & & 13 \\
\hline & SM & & $\mathrm{s}$ & $13 \mathrm{~S}$ & & 3 & 3 & 0 & 13 & 1 & 1 & 1 & 2 & $\leq 0.5$ & 3 & 0 & 13 & 0 & 6 & 1 & 2 \\
\hline & SL & & $\mathrm{U}$ & $\mathrm{O}$ & & $\mathrm{DH}$ & 10 & 1 & & 2 & 12 & 2 & 12 & \begin{tabular}{|c|}
0.6 \\
\end{tabular} & 4 & 0.1 & & 0.5 & 6 & 2 & 9 \\
\hline & BM & & $\mathrm{T}$ & C & & DM & & 2 & & 3 & & 3 & & 0.7 & 1 & 0.2 & & 1 & & 3 & 2 \\
\hline & $\mathrm{BL}$ & & & & & & & 3 & & 4 & & & & 0.8 & 3 & 0.3 & & 1.5 & & 4 & \\
\hline & & & & & & & & 4 & & & & & & $\geq 0.9$ & $1 \geq$ & $\geq 0.4$ & & 2 & & 5 & \\
\hline & & & & & & & & & & & & & & & & & & $\geq 2.5$ & & $\geq 6$ & \\
\hline \multirow[t]{7}{*}{ C. (C.) stelucarum } & $\mathrm{N}$ & 11 & & 11 & 11 & & 11 & & 11 & & 11 & & 11 & & 10 & & 11 & & 9 & & 11 \\
\hline & SM & 1 & $S$ & $11 \mathrm{~S}$ & 11 & $\mathrm{O}$ & 3 & 0 & 9 & 1 & & 1 & 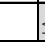 & $\leq 0.5$ & 1 & 0 & 7 & 0 & 2 & 1 & 9 \\
\hline & SL & 5 & $\mathrm{U}$ & $\mathrm{o}$ & & DH & 7 & 1 & 2 & 2 & 5 & 2 & 11 & \begin{tabular}{|c|}
0.6 \\
\end{tabular} & & 0.1 & 4 & 0.5 & 5 & 2 & 2 \\
\hline & BM & 1 & $\mathrm{~T}$ & C & & DM & 1 & 2 & & 3 & 6 & 3 & & 0.7 & 4 & 0.2 & & 1 & 2 & 3 & \\
\hline & BL & 4 & & & & & & 3 & & 4 & & & & 0.8 & 5 & 0.3 & & 1.5 & & 4 & \\
\hline & & & & & & & & 4 & & & & & & $\geq 0.9$ & & $\geq 0.4$ & & 2 & & 5 & \\
\hline & & & & & & & & & & & & & & & & & & $\geq 2.5$ & & $\geq 6$ & \\
\hline \multirow[t]{7}{*}{ C. (C.) tergestinum } & $\mathrm{N}$ & 104 & & 104 & 104 & & 104 & & 103 & & 101 & & 104 & & 98 & & 102 & & 90 & & 103 \\
\hline & SM & & $\mathrm{s}$ & $99 \mathrm{~S}$ & & 50 & & 0 & 102 & 1 & 2 & 1 & 2 & $\leq 0.5$ & & 0 & 6 & 0 & 60 & 1 & 1 \\
\hline & $\mathrm{SL}$ & 98 & $\mathrm{U}$ & 50 & 46 & $6 \mathrm{DH}$ & 21 & 1 & 1 & 2 & 12 & 2 & 54 & 0.6 & 4 & 0.1 & 43 & 0.5 & 19 & 2 & 6 \\
\hline & BM & & $\mathrm{T}$ & C & & DM & 83 & 2 & & 3 & 69 & 3 & 48 & 0.7 & 23 & 0.2 & 37 & 1 & 11 & 3 & 27 \\
\hline & $\mathrm{BL}$ & 6 & & & & & & 3 & & 4 & 18 & & & 0.8 & 34 & 0.3 & 11 & 1.5 & & 4 & 41 \\
\hline & & & & & & & & 4 & & & & & & $\geq 0.9$ & $37 \geq$ & $\geq 0.4$ & 5 & 2 & & 5 & 22 \\
\hline & & & & & & & & & & & & & & & & & & $\geq 2.5$ & & $\geq 6$ & 6 \\
\hline \multirow[t]{7}{*}{ C. (C.) villae } & $\mathrm{N}$ & 18 & & 18 & 18 & & 18 & & 18 & & 18 & & 18 & & 18 & & 18 & & 17 & & 18 \\
\hline & SM & & $\mathrm{s}$ & $17 \mathrm{~S}$ & & $6 \mathrm{O}$ & & 0 & 18 & 1 & & 1 & 12 & $\leq 0.5$ & & 0 & 3 & 0 & 4 & 1 & \\
\hline & SL & 18 & $\mathrm{U}$ & 10 & & $\mathrm{DH}$ & 7 & 1 & & 2 & 2 & 2 & 6 & \begin{tabular}{|l|}
0.6 \\
\end{tabular} & & 0.1 & 8 & 0.5 & 5 & 2 & 1 \\
\hline & BM & & $\mathrm{T}$ & C & & $\mathrm{DM}$ & 11 & 2 & & 3 & 14 & 3 & & 0.7 & 10 & 0.2 & 7 & 1 & 8 & 3 & 8 \\
\hline & BL & & & & & & & 3 & & 4 & 2 & & & 0.8 & 4 & 0.3 & & 1.5 & & 4 & 6 \\
\hline & & & & & & & & 4 & & & & & & $\geq 0.9$ & 4 & $\geq 0.4$ & & 2 & & 5 & 3 \\
\hline & & & & & & & & & & & & & & & & & & $\geq 2.5$ & & $\geq 6$ & \\
\hline \multirow[t]{7}{*}{ C. (C.) waldemari } & $\mathrm{N}$ & 50 & & 50 & 50 & & 50 & & 50 & & 46 & & 50 & & 46 & & 50 & & 43 & & 50 \\
\hline & SM & 11 & S & $50 \mathrm{~S}$ & & $\mathrm{O}$ & & 0 & 48 & 1 & 3 & 1 & 2 & $\leq 0.5$ & 2 & 0 & 22 & 0 & 42 & 1 & 1 \\
\hline & SL & 39 & $\mathrm{U}$ & $\mathrm{O}$ & & $5 \mathrm{DH}$ & 24 & 1 & 2 & 2 & 11 & 2 & 28 & \begin{tabular}{|c|}
0.6 \\
\end{tabular} & 7 & 0.1 & 18 & 0.5 & 1 & 2 & 22 \\
\hline & BM & & $\mathrm{T}$ & C & & $7 \mathrm{DM}$ & 26 & 2 & & 3 & 26 & 3 & 20 & 0.7 & 7 & 0.2 & 4 & 1 & & 3 & 20 \\
\hline & $\mathrm{BL}$ & & & & & & & 3 & & 4 & 6 & & & 0.8 & 18 & 0.3 & 4 & 1.5 & & 4 & 4 \\
\hline & & & & & & & & 4 & & & & & & $\geq 0.9$ & $12=$ & $\geq 0.4$ & 2 & 2 & & 5 & 3 \\
\hline & & & & & & & & & & & & & & & & & & $\geq 2.5$ & & $\geq 6$ & \\
\hline
\end{tabular}


Table 5. GenBank voucher (H3).

\begin{tabular}{|c|c|c|c|}
\hline Taxon & Location & Isolate & GenBankVoucher (H3) \\
\hline Cochl. (Clessiniella) anomphale & Eisenkappel, Austria & EZO034 & KP408278 \\
\hline Cochl. (Clessiniella) stelucarum & Anduins, Italy & EZ0102 & KX120829 \\
\hline Cochl. (Clessiniella) stelucarum & Cornino, Italy & EZO104 & KX120830 \\
\hline Cochl. (Clessiniella) stelucarum & Mt Bernadia, Italy & EZO107 & KX120831 \\
\hline Cochl. (Clessiniella) tergestinum & Val Rosandra, Italy & EZO840 & KP408283 \\
\hline Cochl. (Clessiniella) tergestinum & Rakow Skocjan, Slovenia & EZ0838 & KP408282 \\
\hline Cochl. (Clessiniella) tergestinum & Drivenik spring, Croatia & WdM6714 & KP408281 \\
\hline Cochl. (Clessiniella) tergestinum & Jakačici, Croatia & WdM6699 & KP408280 \\
\hline Cochl. (Clessiniella) tergestinum & Rasko Polje, Croatia & EZ0813 & KP408279 \\
\hline Cochl. (Clessiniella) villae & Perarolo, Italy & EZ0891 & KP408284 \\
\hline Cochl. (Clessiniella) waldemari & Mt Klek, Croatia & EZ0904 & KP408289 \\
\hline Cochl. (Clessiniella) waldemari & Benete, Slovenia & WdM6805 & KP408288 \\
\hline Cochl. (Clessiniella) waldemari & Vrsic Pass, Slovenia & EZ0896 & KP408287 \\
\hline Cochl. (Clessiniella) waldemari & Tisovec, Slovenia & WdM6749 & KP408286 \\
\hline Cochl. (Clessiniella) waldemari & Polog-E of Čepovan, Slovenia & WdM6842 & KP408285 \\
\hline Cochl. (Clessiniella) waldemari & Mt Klek, Croatia & EZ0905 & KX120837 \\
\hline Cochl. (Cochlostoma) septemspirale & Plitvice, Croatia & WdM3031 & KP408291 \\
\hline
\end{tabular}

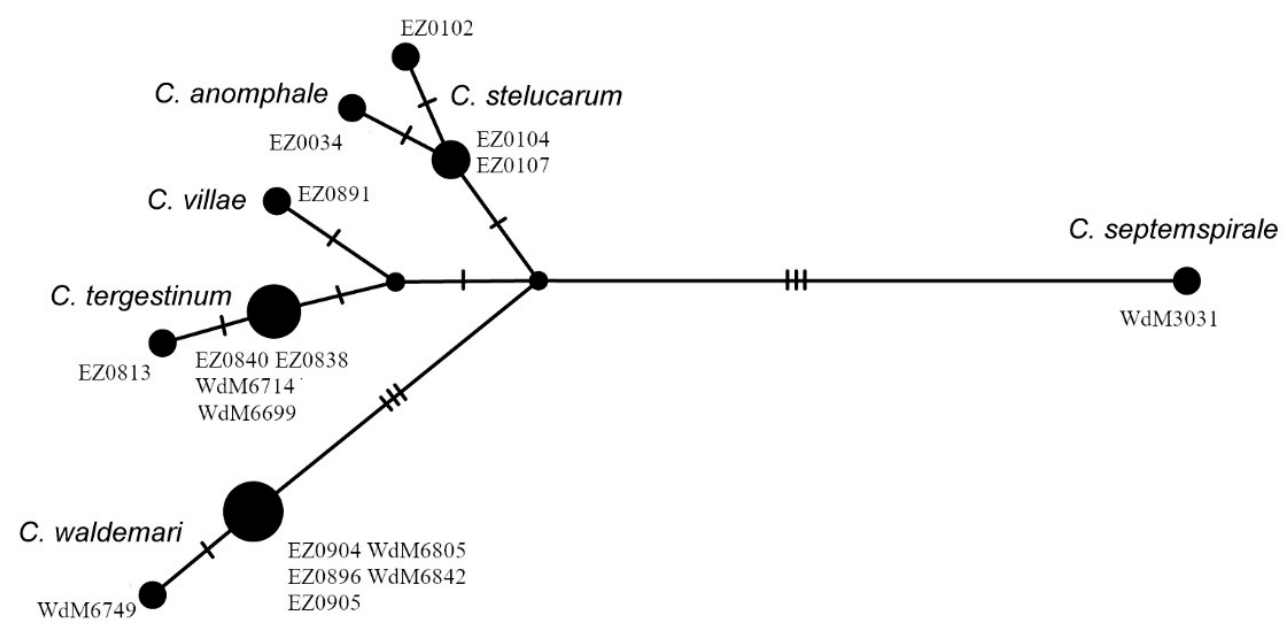

Fig. 7. Statistical parsimony network based on Histone $\mathrm{H} 3$ sequences of species in the subgenus Clessiniella Zallot et al., 2015. Cochlostoma (Cochlostoma) septemspirale (Razoumowsky, 1789) was used as an outgroup. For sample IDs we refer to Table 5. 


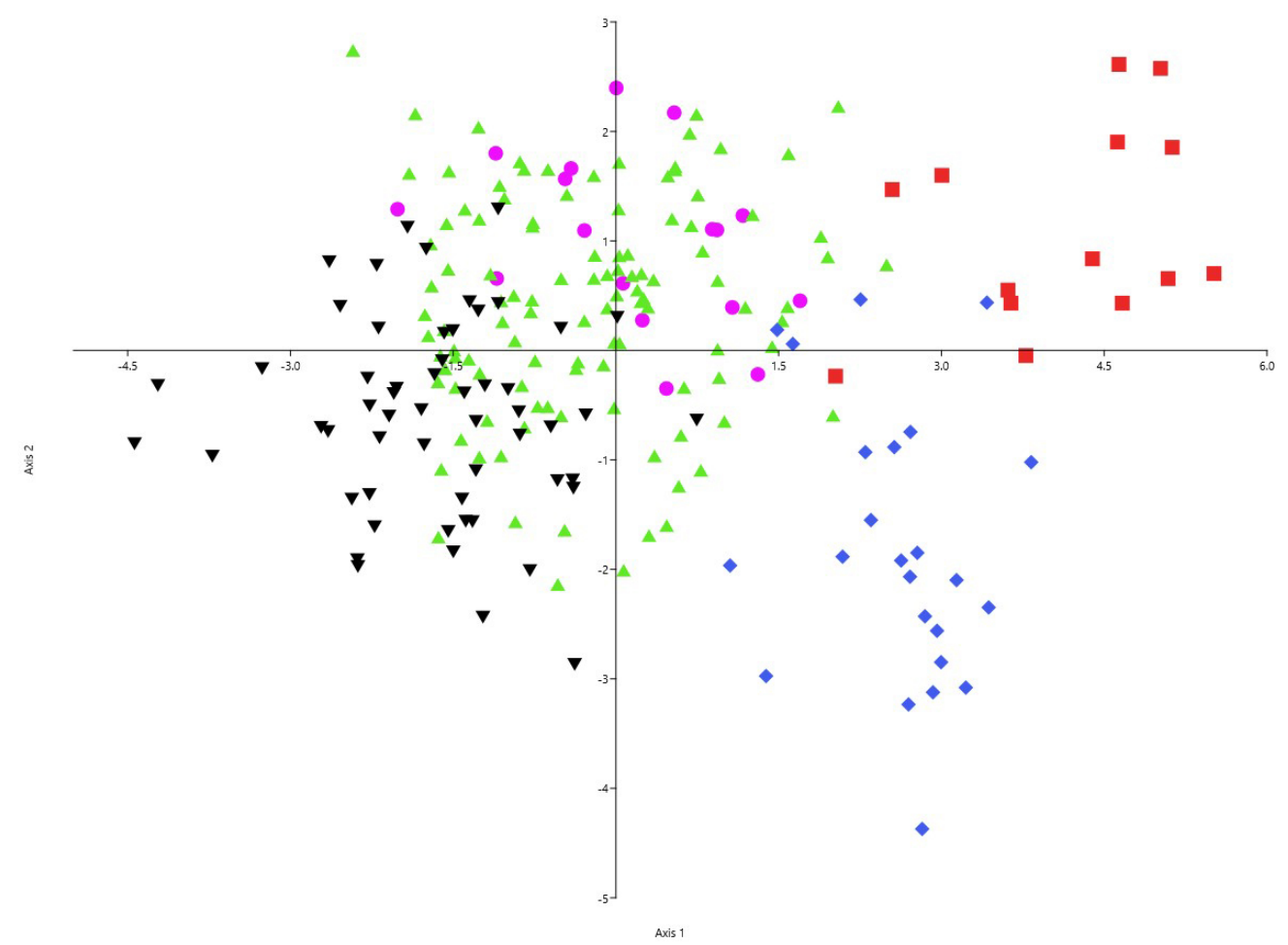

\begin{tabular}{|l|r|}
\hline Box's M test & \\
\hline Box's M: & 5248,1 \\
\hline F: & 19,065 \\
\hline$d f 1 ; d f 2:$ & $220 ; 9788.2$ \\
\hline$p$ (equal var-covar): & 0 \\
\hline Monte Carlo p: & 0,0185 \\
\hline
\end{tabular}

\begin{tabular}{|l|r|r|r|r|}
\cline { 2 - 5 } \multicolumn{1}{l|}{ Loading of the variables } & \multicolumn{1}{l|}{ Axis 2 } & \multicolumn{1}{l|}{ Axis 3 } & \multicolumn{1}{l|}{ Axis 4 } \\
\hline number of whorls & 2,2306 & 0,82113 & 1,3244 & 3,4638 \\
\hline height & $-1,2809$ & $-0,31444$ & $-0,7091$ & $-2,2692$ \\
\hline max width & 2,7766 & $-1,258$ & 2,2366 & 2,7708 \\
\hline H Apert/H & $-12,114$ & $-8,7672$ & 9,7403 & 45,088 \\
\hline w roundiness & $-13,238$ & 8,7285 & $-18,63$ & 5,3462 \\
\hline ribs inclination & 0,26508 & $-0,15718$ & 0,032586 & $-0,18707$ \\
\hline Aperture inclination & 0,072479 & 0,075594 & $-0,088501$ & $-0,03028$ \\
\hline Ribs/mm 1st whorl & 0,021996 & 0,1147 & 0,095936 & $-0,19779$ \\
\hline Ribs/mm 4th whorl & $-0,1459$ & 0,089018 & 0,50915 & $-0,049045$ \\
\hline Protoconch size & $-3,1587$ & $-8,639$ & $-3,3185$ & 9,3502 \\
\hline
\end{tabular}

\begin{tabular}{|l|r|r|r|r|r|r|}
\cline { 2 - 8 } Confusion matrix & A & S & T & V & W & Total \\
\hline A (anomphale) & 14 & 0 & 3 & 0 & 0 & 17 \\
\hline S (stelucarum) & 0 & 13 & 0 & 1 & 0 & 14 \\
\hline T (tergestinum) & 10 & 1 & 79 & 3 & 18 & 111 \\
\hline V (villae) & 0 & 2 & 1 & 21 & 0 & 24 \\
\hline W (waldemari) & 1 & 0 & 8 & 0 & 47 & 56 \\
\hline Total & 25 & 16 & 91 & 25 & 65 & 222 \\
\hline
\end{tabular}

Fig. 8. Discriminant analysis based on shell features of the species in Clessiniella Zallot et al., 2015. Violet dots $=$ Cochlostoma $($ Clessiniella $)$ anomphale Boeckel, 1939; red squares $=$ Co.$(C l$.$) stelucarum$ sp. nov.; green triangles $=C o .(C l$.$) tergestinum ($ Westerlund, 1878); blue diamonds $=C o .(C l$.$) villae$ $($ Strobel, 1851); black triangles $=C o .(C l$.$) waldemari (A.J. Wagner, 1897). Analysis performed with$ the software 'Past' (https://palaeo-electronica.org). 


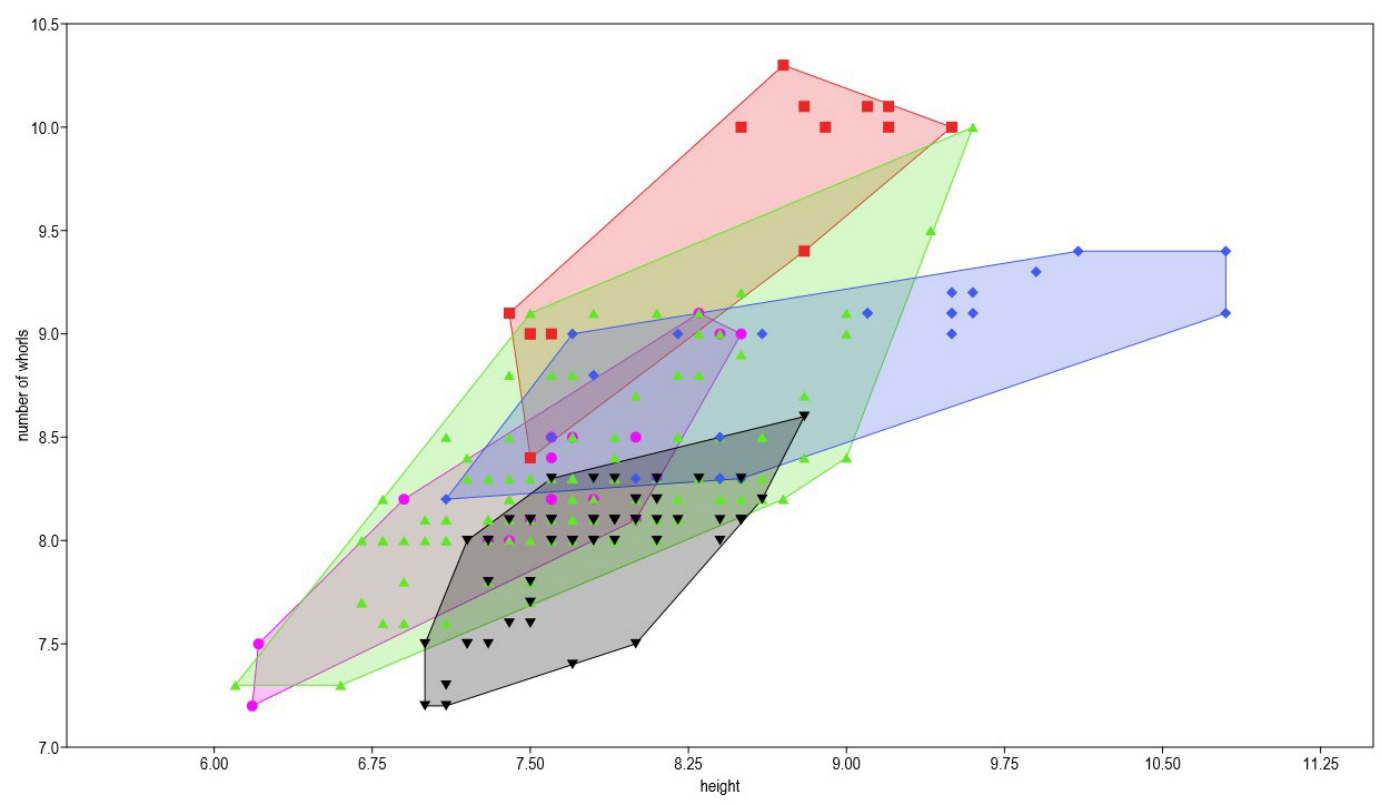

Fig. 9. Number of whorls vs height of the species in Clessiniella Zallot et al., 2015. Violet dots = Cochlostoma (Clessiniella) anomphale Boeckel, 1939; red squares $=$ Co. $(C l$.) stelucarum sp. nov.; green triangles $=C o .(C l$.$) tergestinum$ (Westerlund, 1878); blue diamonds $=C o .(C l$.$) villae (Strobel,$ 1851); black triangles $=C o$. $(C l$. $)$ waldemari (A.J. Wagner, 1897). Analysis performed with the software 'Past' (https://palaeo-electronica.org).

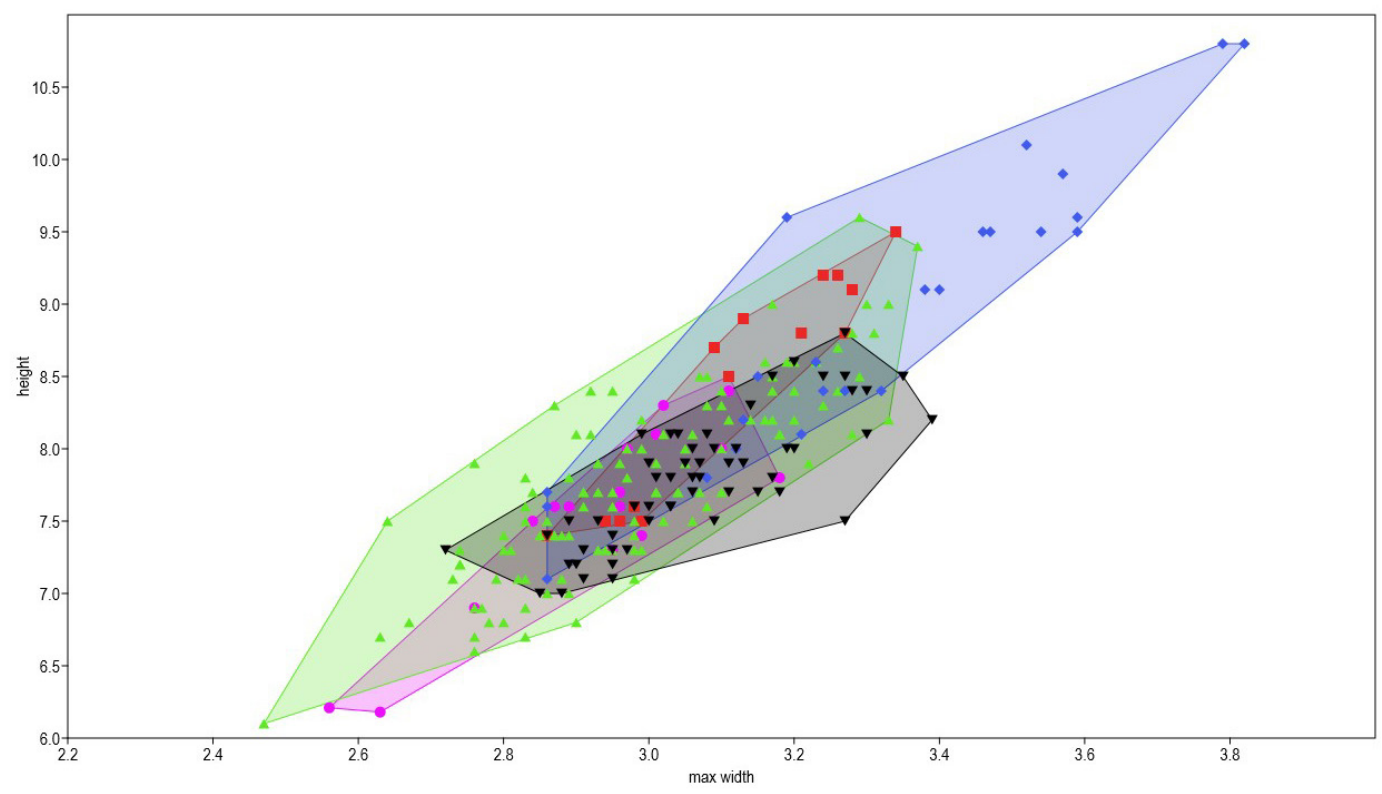

Fig. 10. Width of the $1^{\text {st }}$ whorl vs height (bottom) of the species in Clessiniella Zallot et al., 2015. Violet dots $=$ Cochlostoma $($ Clessiniella $)$ anomphale Boeckel, 1939; red squares $=$ Co.$(C l$.$) stelucarum$ sp. nov.; green triangles $=C o$. $(C l$. $)$ tergestinum (Westerlund, 1878); blue diamonds $=C o .(C l$.$) villae$ (Strobel, 1851); black triangles $=C o .(C l$.$) waldemari (A.J. Wagner, 1897). Analysis performed with$ the software 'Past' (https://palaeo-electronica.org). 


\title{
DNA analysis
}

Female specimens of each species were used for DNA extraction. A Histone fragment (H3) was the only DNA marker we could successfully amplify in the 5 species. New H3 sequences were deposited in GenBank (KX120829-31 and KX120837, see Table 5). They were aligned with the sequences of Clessiniella published by Zallot et al. (2015) (Fig. 3). As Zallot et al. (2015) have found that Cochlostoma s. str. is the sister clade of Clessiniella in the $\mathrm{H} 3$ tree, a sequence of Co. (Co.) septemspirale (Razoumowsky, 1789) was used as outgroup. Because of the low resolution, the phylogeny does not show a tree-like structure. Therefore, we illustrated in Fig. 7 the relationships by a statistical parsimony network (Clément et al. 2002) calculated by Popart ver. 1.7. (Leigh \& Bryant 2015). It suggests that $C o$. (Cl.) stelucarum sp. nov. might be the closest relative of Co. (Cl.) anomphale Boeckel, 1939 and that Co. $(\mathrm{Cl}$.) tergestinum (Westerlund, 1878$)$ and $\mathrm{Co}$. $(\mathrm{Cl}$.) villae (Strobel, 1851) as closely related sister species. Because the limited number of specimens analyzed (only 1 specimen in $\mathrm{Co}$. (Cl.) anomphale and $\mathrm{Co}$. (Cl.) villae), however, further and more extensive molecular research would be needed.

\section{Taxonomy}

\author{
Subclass Caenogastropoda Cox, 1960 \\ Order Architaenioglossa Haller, 1892 \\ Superfamily Cyclophoroidea J.E. Gray, 1847 \\ Family Cochlostomatidae Kobelt, 1902 \\ Genus Cochlostoma Jan, 1830
}

Subgenus Clessiniella Zallot, Groenenberg, De Mattia, Fehér \& Gittenberger, 2015

Clessiniella Zallot, Groenenberg, De Mattia, Fehér \& Gittenberger, 2015: 80.

\section{Type species}

Pomatias maculatum var. villae Strobel, 1851, by original designation.

\section{Remarks}

The classification follows MolluscaBase (2021) except for the subgeneric name. Contrary to ICZN Art. 10.4, Neglecta Wagner, 1897 is used in MolluscaBase as a senior synonym of Clessiniella. Wagner (1897: 6 [570]) introduced Neglecta as "Formenkreis Neglecta". It cannot be denied that the German term Formenkreis is ".. a term such as "superspecies"”. As a consequence, according to the ICZN ruling, Neglecta ".. is not deemed to be a genus-group name" (ICZN Art. 10.4, see also Zallot et al. 2015). Strobel (1851) and De Betta \& Martinati (1855) named this entity Pomatias maculatum var. Villae - but referring to Pomatias maculatus Draparnaud, 1805 (=Co. (Co.) septemspirale).

\section{Diagnosis of the subgenus}

SHell. Ribbed teleoconch with straight ribs, variable in strength and shape. The columellar lobe is curved backwards, covering the umbilicus. The teleoconch spots (if present) form 2 rows on the whorls (often, a $3^{\text {rd }}$ row of spots is visible on the body whorl): the upper one close to the suture with rectangular spots abapically elongated; the lower one roughly $1 / 3$ up on the whorls (Fig. 12). In some populations the 2 rows of spots merge and there is a continuous vertical darker band on the whorls. The protoconch is dull and robust with fine riblets starting after 1.2 whorls or more from the apex; it is transparent and fragile or the ribbing starts close to the apex in other subgenera.

Female genitalia. There is an apical connection of the pedunculus to the bursa copulatrix, a long seminal receptacle (normally club-shaped), which runs parallel to the pedunculus. The junction of the uterus gland is situated close to the connection between the pedunculus and the distal oviduct. This 

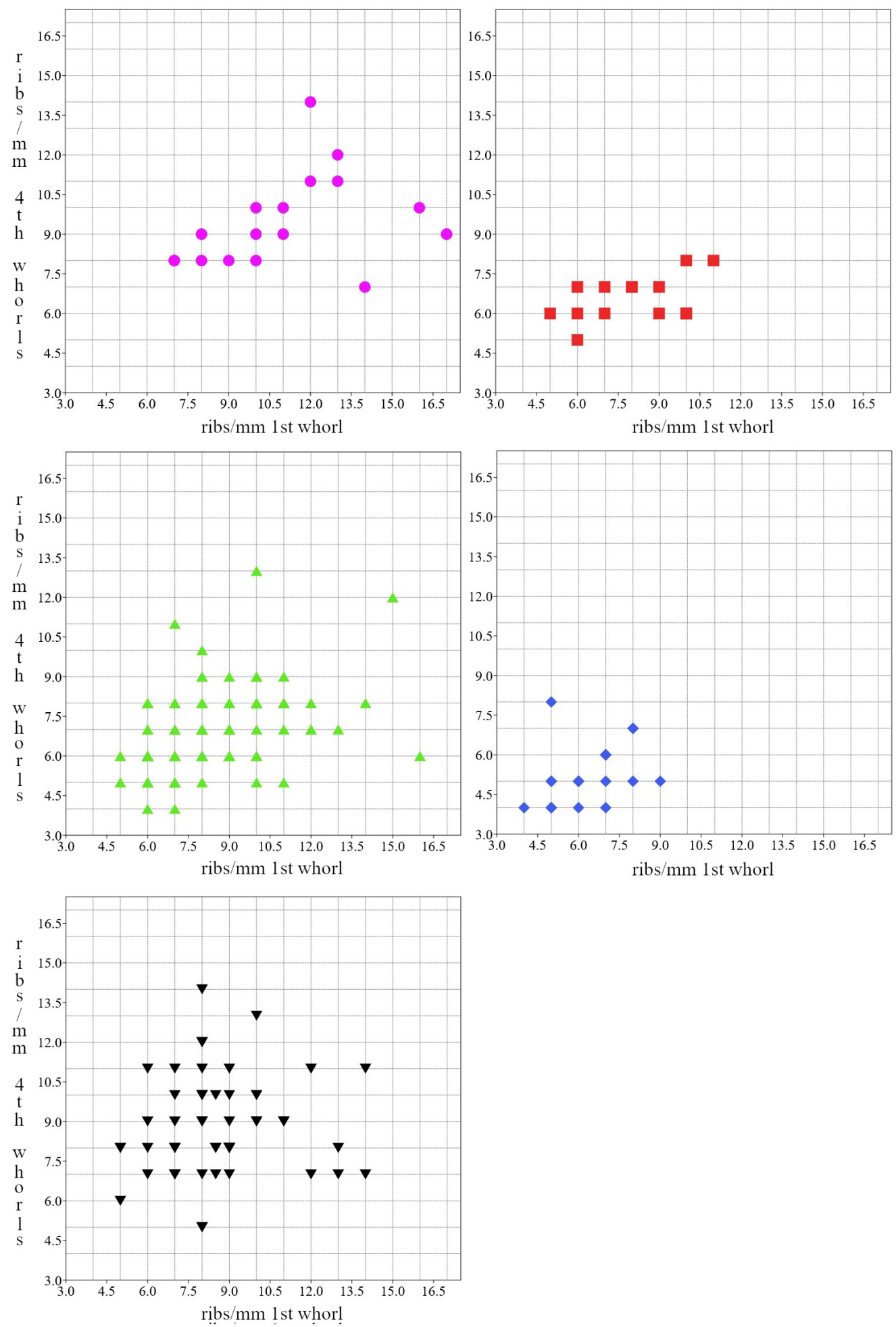

Fig. 11. Ribs per mm on the $1^{\text {st }}$ whorl vs ribs on the $4^{\text {th }}$ whorls of the species in Clessiniella Zallot et al., 2015. Violet dots = Cochlostoma (Clessiniella) anomphale Boeckel, 1939; red squares = Co. $(\mathrm{Cl}$.$) stelucarum sp. nov.; green triangles =C o .(C l$.$) tergestinum ($ Westerlund, 1878$)$; blue diamonds $=C o .(C l$.$) villae (Strobel, 1851); black triangles =C o .(C l$.$) waldemari (A.J. Wagner, 1897). Analysis$ performed with the software 'Past' (https://palaeo-electronica.org). 
combination of characters in the female genitalia is most diagnostic to distinguish Clessiniella from the other subgenera.

Male genitalia. Apart from its apical part, the penial spermiduct is tortuous. The body spermiduct is protruding (often almost detached from the body) more than in the other subgenera and either straight or more or less tortuous. The posterior side of the penis is wrinkled.

\section{Identification key for the species}

1. Body whorl inflated shortly before the lip (Fig. 4A: OB). In the female genitalia, simplified loop path with 1-2 loops

Co. (Cl.) anomphale Boeckel, 1939

- Body whorl not inflated before the lip (Fig. 4A: FF, AF, II)

2. In the female genitalia, the loops of the visceral oviduct are $1-2$

Co. (Cl.) stelucarum sp. nov.

- In the female genitalia, the loops of the visceral oviduct are more than 2

3. In the male genitalia, the body spermiduct is twisted

- In the male genitalia, the body spermiduct is straight

Co. (Cl.) waldemari (Wagner, 1897)

4. Ribs of the shell widely spaced and barely inclined, protoconch relatively small (Fig. 13). Slim, club-shaped seminal receptacle in the female genitalia

Co. (Cl.) villae (Strobel, 1851)

- Ribs less widely spaced and more inclined, relatively larger protoconch. Less slim seminal receptacle

Co. (Cl.) tergestinum (Westerlund, 1878)
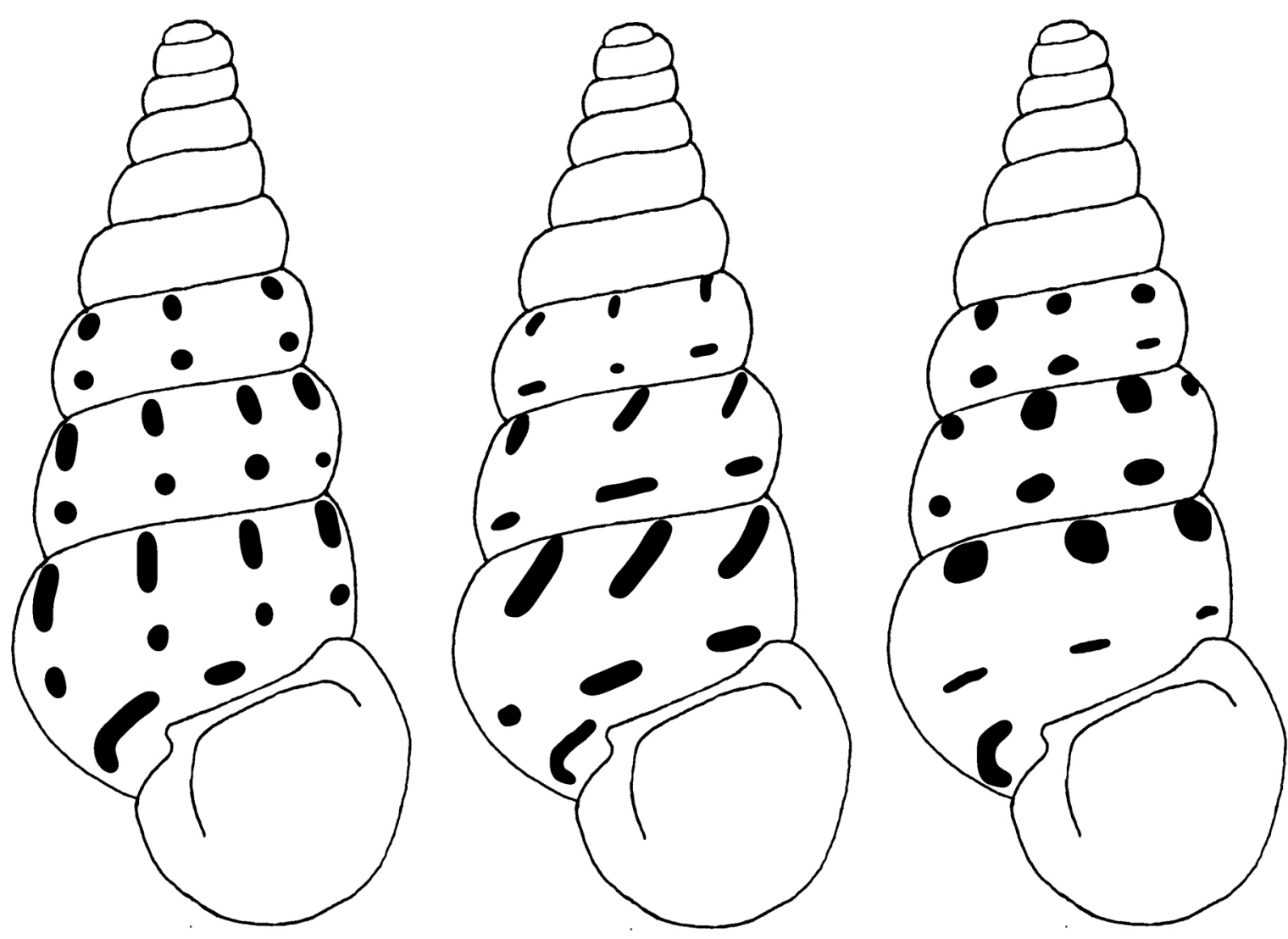

Fig. 12. Most common spot patterns on the shells of the species of Clessiniella Zallot et al., 2015. 


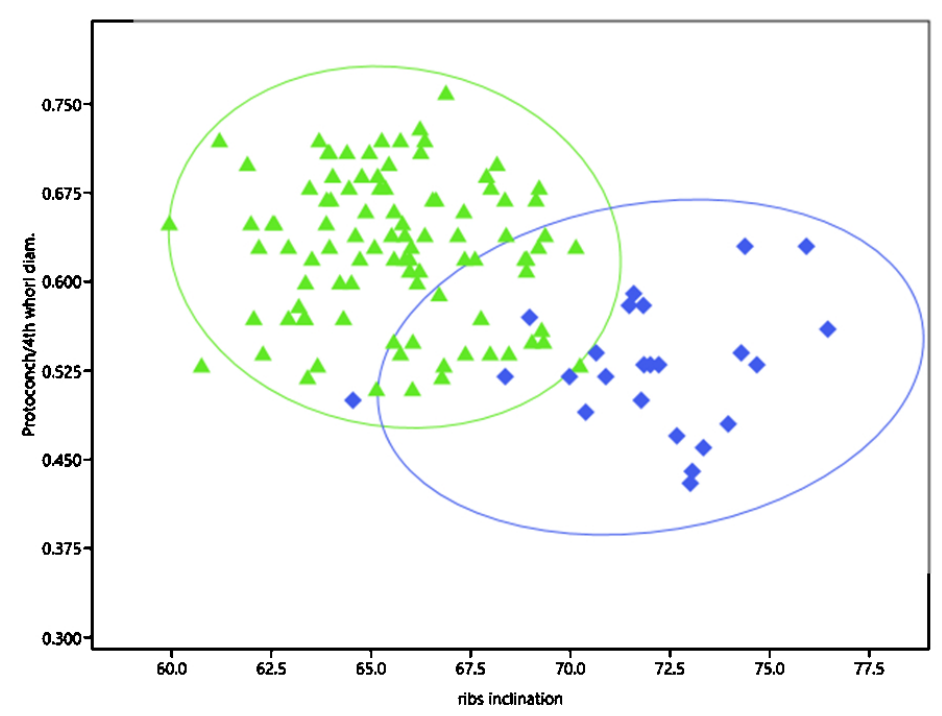

Fig. 13. Shell characters (ribs inclination and relative size of the protoconch) which could be helpful to distinguish Cochlostoma (Clessiniella) villae (Strobel, 1851) (blue diamonds) from Co. (Cl.) tergestinum (Westerlund, 1878) (green triangles).

Cochlostoma (Clessiniella) villae (Strobel, 1851)

Figs 1, 3C, 14D, 15D, 16D, 17-21

Pomatias maculatum var. villae Strobel, 1851: 17 ("Mizzole presso Verona").

Pomatias maculatum var. villae De Betta \& Martinati, 1855: 74 ("Prov. Verona, Mizzole").

Cochlostoma (Auritus) villae - Alzona 1971: 22.

Cochlostoma villae - Welter-Schultes 2012: 102.

\section{Diagnosis}

The last whorl, approaching the aperture, hardly enlarges (Fig. 4A: FF). Very prominent and widely spaced teleoconch ribs, barely inclined in respect to the vertical axis. Spots almost always visible. The protoconch is relatively small. The apertural lip poorly developed. Visceral oviduct with convoluted loops; thin seminal receptacle. Straight body spermiduct.

\section{Type material}

\section{Syntypes}

ITALY • 1 P; "Pomatias villae Spinel. Mizzole. Villa 49" [label of the original material] [Veneto, Verona, Mizzole] [thus, it could be interpreted as collected in 1849 by Villa]; PU1610-C1 (Fig. 19) • 2 $\widehat{\partial}$; same label data as for preceding; PU1610-C2, PU1610-C3 (Fig. 20).

Other material (fresh specimens from the type locality have also been analyzed)

ITALY • Altissimo; $45.61511^{\circ} \mathrm{N}, 11.25225^{\circ} \mathrm{E}$; 2009; Zallot leg.; EZ0883 • Arqua' Petrarca, surroundings; 45.26194 ${ }^{\circ} \mathrm{N}, 11.69623^{\circ} \mathrm{E}$; 1999 ; Zallot leg.; EZ0895 • Bolca; $45.59389^{\circ} \mathrm{N}, 11.20972^{\circ} \mathrm{E}$; 2010; Zallot leg.; EZ0882 • Ca'Gottolo; $45.58921^{\circ} \mathrm{N}, 10.90787^{\circ}$ E; Jun. 2010; Scarlassara leg.; EZ1015 • Cornedo Vicentino-Cereda; $45.60667^{\circ} \mathrm{N}, 11.36833^{\circ} \mathrm{E}$; 1999; Zallot leg.; EZ0887 • Covoli di Velo; $45.60889^{\circ} \mathrm{N}$, 11.12083 E; 2010; Zallot leg.; EZ0879 • Ferrazza; $45.62137^{\circ}$ N, $11.19538^{\circ}$ E; Apr. 2010; Zallot leg.; 
EZ0881 • Fumane-Molina; $45.56942^{\circ}$ N, $10.89856^{\circ}$ E; Jun. 2010; Scarlassara leg.; EZ0936 • Lago di Fimon; 45.46972 ${ }^{\circ} \mathrm{N}, 11.54^{\circ} \mathrm{E}$; 2009; Zallot leg.; EZ0892 • Lumignano; $45.45607^{\circ} \mathrm{N}, 11.57914^{\circ} \mathrm{E}$; 2010; Zallot leg.; EZ0893 • Mizzole; $45.48662^{\circ}$ N, $11.05524^{\circ}$ E; Apr. 2010; Zallot leg.; EZ0878 • Monte delle Piume; $45.35477^{\circ} \mathrm{N}, 11.45641^{\circ}$ E; May 2010; G. Zallot leg.; EZ0889 • Mt Rusta; $45.28735^{\circ} \mathrm{N}$, $11.68563^{\circ} \mathrm{E}$; 2002; Zallot leg.; EZ0894 • Novale-Valdagno; $45.66528^{\circ} \mathrm{N}, 11.29528^{\circ} \mathrm{E}$; 2000; Zallot leg.; EZ0884 • Perarolo dei Monti Berici, $45.47694^{\circ} \mathrm{N}, 11.50056^{\circ} \mathrm{E}$; 1999, Zallot leg.; EZ0891 • Priabona; 45.63389 $9^{\circ}$ N, $11.37417^{\circ}$ E; 2000; Zallot leg.; EZ0888 • San Giovanni di Zovencedo; $45.42833^{\circ} \mathrm{N}$, $11.49417^{\circ} \mathrm{E}$; 1999 ; Zallot leg.; EZ0890 • Tregnago-Finetti; $45.52866^{\circ} \mathrm{N}, 11.1806^{\circ} \mathrm{E}$; 2005; Zallot leg.; EZ0880 • Valdagno; $45.64991^{\circ} \mathrm{N}, 11.30079^{\circ} \mathrm{E}$; 2000; Zallot leg.; EZ0885.

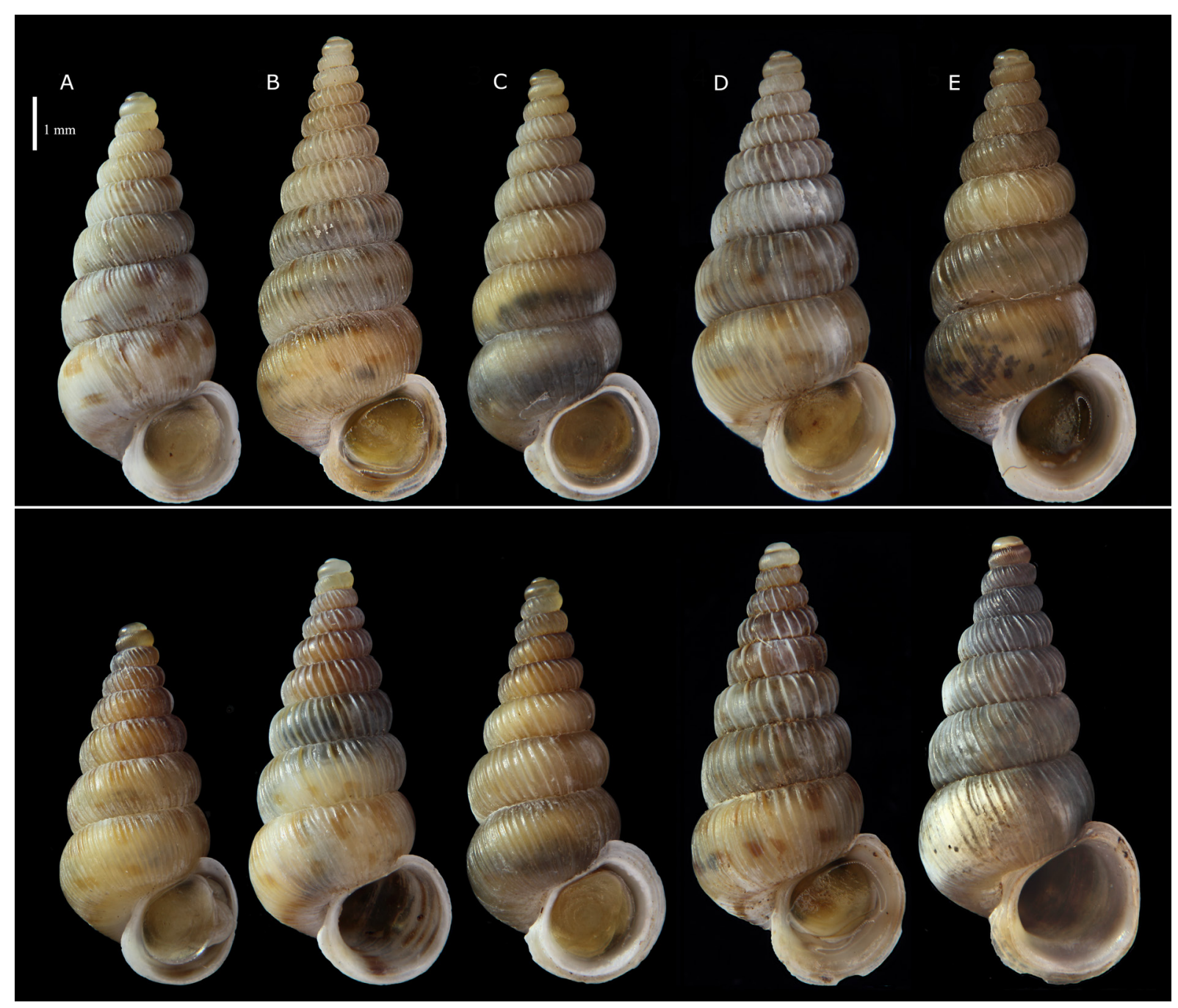

Fig. 14. The five species of Clessiniella Zallot et al., 2015. A-D. Picture of a female (top) and male (bottom) specimen from the type localities. A. Cochlostoma (Clessiniella) anomphale Boeckel, 1939. Vellach, Austria (EZ0991). B. Co. (Cl.) stelucarum sp. nov., Cornino, Italy (EZ0104). C. Co. (Cl.) tergestinum (Westerlund, 1878), Val Rosandra, Italy (EZ0840). D. Co. (Cl.) villae (Strobel, 1851), Mizzole, Italy (EZ0878). E. Specimens from a locality close to the type one: $\mathrm{Co}$. (Cl.) waldemari (A.J.Wagner, 1897), Mt Klek, Croatia (EZ0905). 


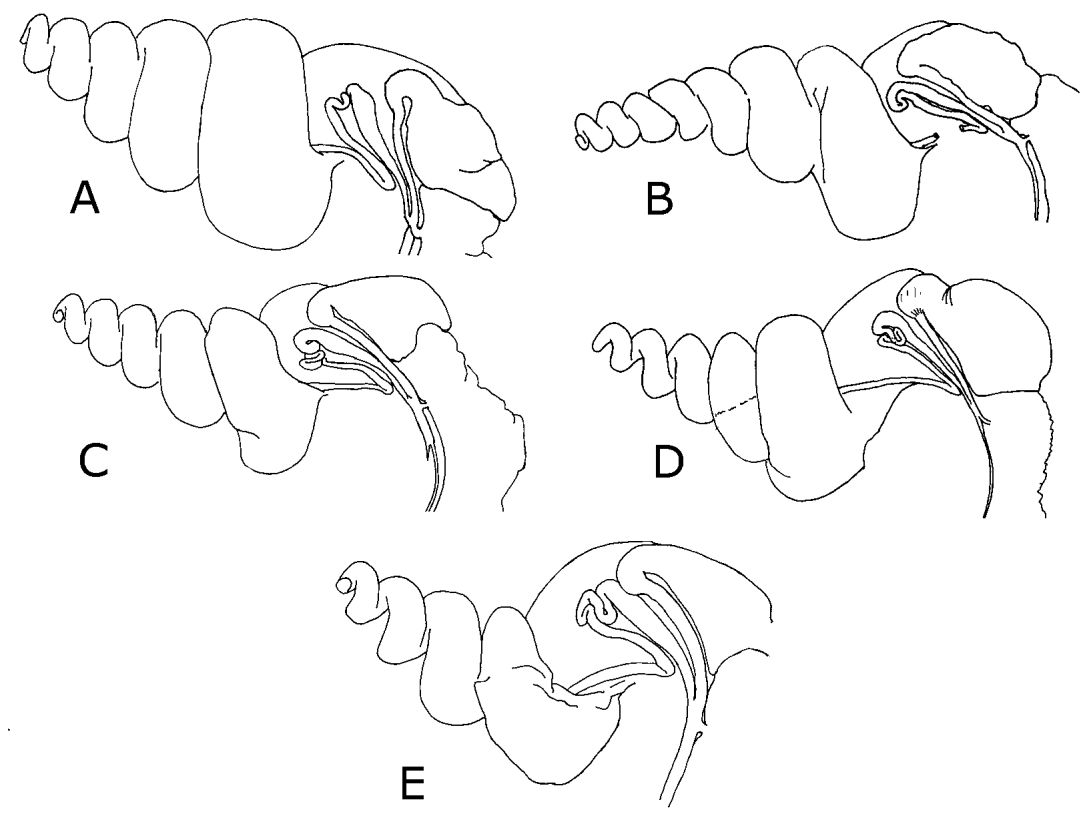

Fig. 15. Comparison of female genitalia. A. Cochlostoma (Clessiniella) anomphale Boeckel, 1939. Vellach, Austria (EZ0991). B. Co. (Cl.) stelucarum sp. nov., Cornino, Italy (EZ0104). C. Co. (Cl.) tergestinum (Westerlund, 1878), Val Rosandra, Italy (EZ0840). D. Co. (Cl.) villae (Strobel, 1851), Mizzole, Italy (EZ0878). E. Co. (Cl.) waldemari (A.J.Wagner, 1897), Mt Klek, Croatia (EZ0905).

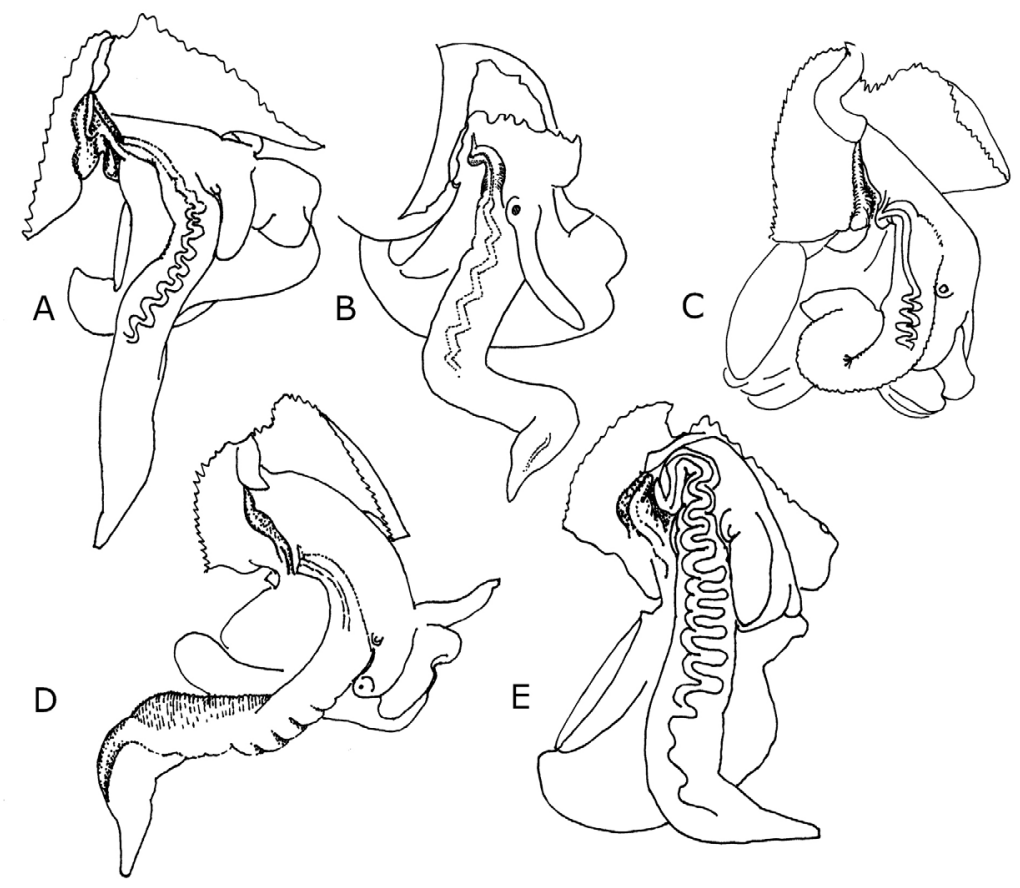

Fig. 16. Comparison of male genitalia. A. Cochlostoma (Clessiniella) anomphale Boeckel, 1939. Vellach, Austria (EZ0991). B. Co. (Cl.) stelucarum sp. nov., Cornino, Italy (EZ0104). C. Co. (Cl.) tergestinum (Westerlund, 1878), Val Rosandra, Italy (EZ0840). D. Co. (Cl.) villae (Strobel, 1851), Mizzole, Italy (EZ0878). E. Co. (Cl.) waldemari (A.J.Wagner, 1897), Tisovec, Croatia (WdM6750). 


\section{Description}

SHELL. The teleoconch whorls have whitish, very prominent, rather straight and widely spaced ribs on the upper whorls (see Fig. 11), but smooth specimens are found occasionally (see https://www.naturamediterraneo.com/forum/topic.asp?TOPIC_ID=43816).

LIP. Moderately developed, and more or less flattened by the internal callus of the aperture. It gently curves backwards at the columellar side, starting rather far from the upper part of the body whorl. On lateral view, the body whorl, approaching the aperture, in most of the specimens barely enlarging (Fig. 4A: FF, Table 3).

Female genitalia. There is a long and relatively thin (as compared to the other species of Clessiniella; see Table 5) seminal receptacle with a curved apex.

Male genitalia. The penis is long and inflated. The penial spermiduct is slightly twisted and occupies only the central part of the penis. There is a clearly protruding but straight body spermiduct.

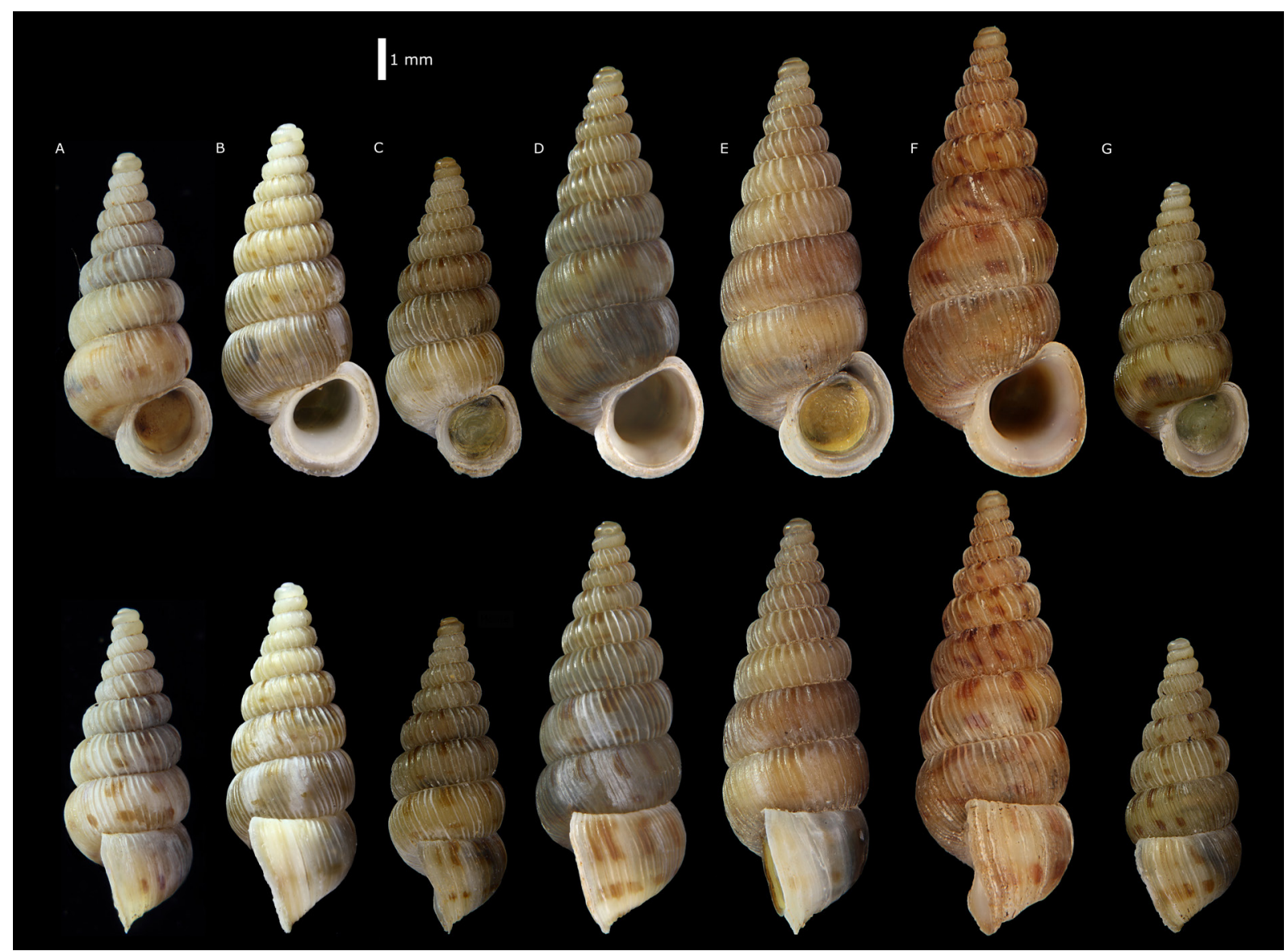

Fig. 17. Shell variability in Cochlostoma (Clessiniella) villae (Strobel, 1851). A. Mizzole, Italy (EZ0878). B. Covoli di Velo, Italy (EZ0879). C. Ferrazza, Italy (EZ0881). D. Mt delle Piume, Italy (EZ0889). E. Perarolo, Italy (EZ0891). F. Lumignano, Italy (EZ0893). G. Ca' Gottolo, Italy (EZ1015). 


\section{Distribution}

The species inhabits a relatively small area at the foothills of the eastern Alps. It can also be found on the hills stretching down the Venetian flatland of the "pianura Veneta" (Colli Berici and Colli Euganei). It has been found sympatric with Cochlostoma (Cochlostoma) septemspirale and Cochlostoma (Eupomatias) philippianum in some localities of the province of Verona (Fernando Scarlassara, personal communication).

\section{Habitat preference}

The snails are found on rocky cliffs as well as on smaller boulders and dry stone walls in woody places.

\section{Remarks}

The range of this species is far from the almost continuous area in the east where the other Clessiniella species are known from. The species has been reported (and still is in the checklist of the Italian fauna - www.faunaitalia.it) as Cochlostoma villae (De Betta \& Martinati, 1855) but Bank (1988) found that Strobel (1851) described it as Pomatias maculatum var. villae Spinelli, four years before De Betta \& Martinati (1855). The description provided by Strobel (1851) is poor: "Varieta' generalmente maggiore, coi giri della spira piu' gonfi e colle coste trasversali piu' regolari, e saglienti" = "variety generally bigger, with more rounded whorls and more regular and strong ribs" and follows " $P$. Villae Spinelli in

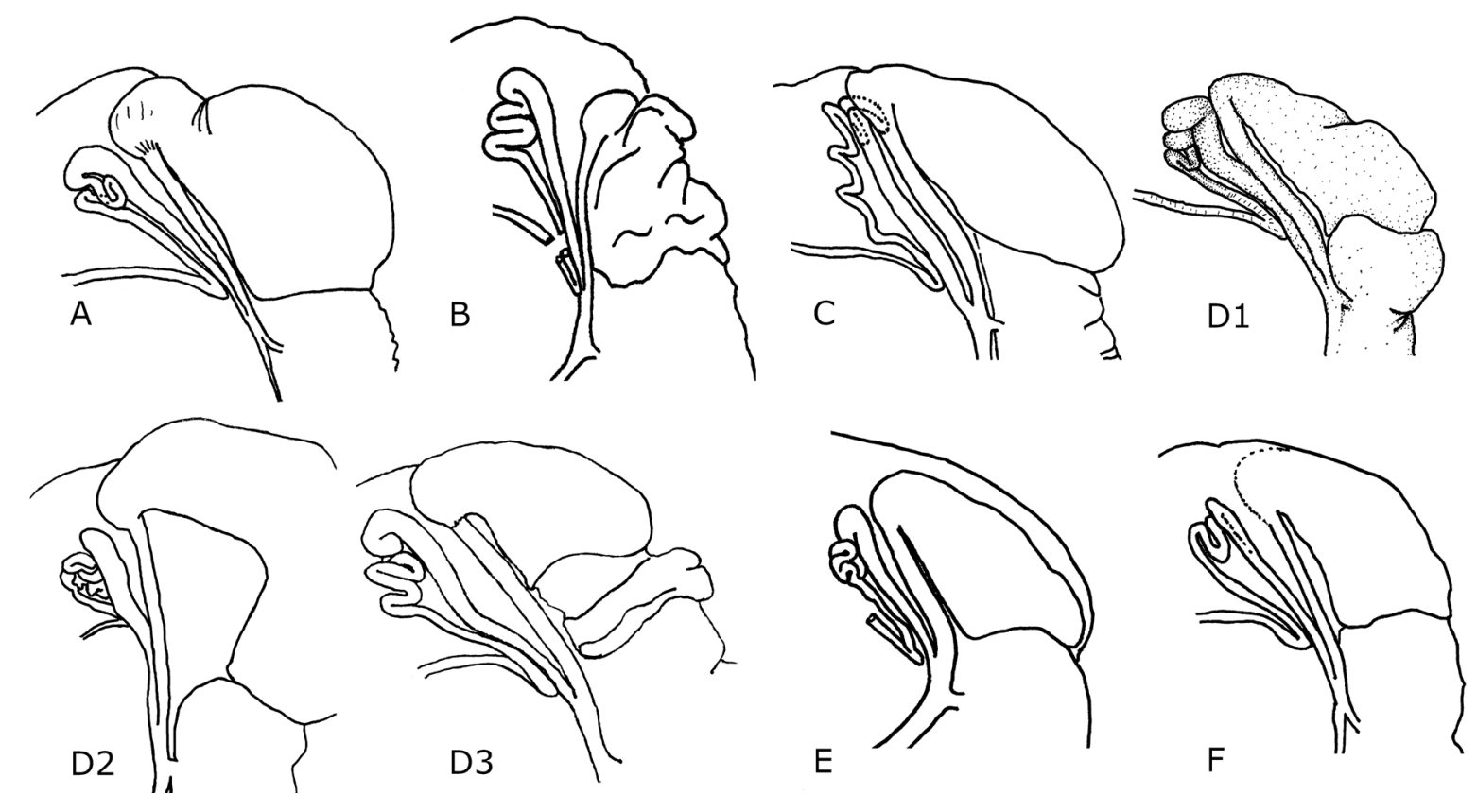

Fig. 18. Variation of the female genitalia in Cochlostoma (Clessiniella) villae (Strobel, 1851). A. Mizzole, Italy (EZ0878). B. Ferrazza, Italy (EZ0881). C. Mt delle Piume, Italy (EZ0889). D1D3. Perarolo, Italy (EZ0891). E. Mt Rusta, Italy (EZ0894). F. Ca' Gottolo, Italy (EZ1015). 


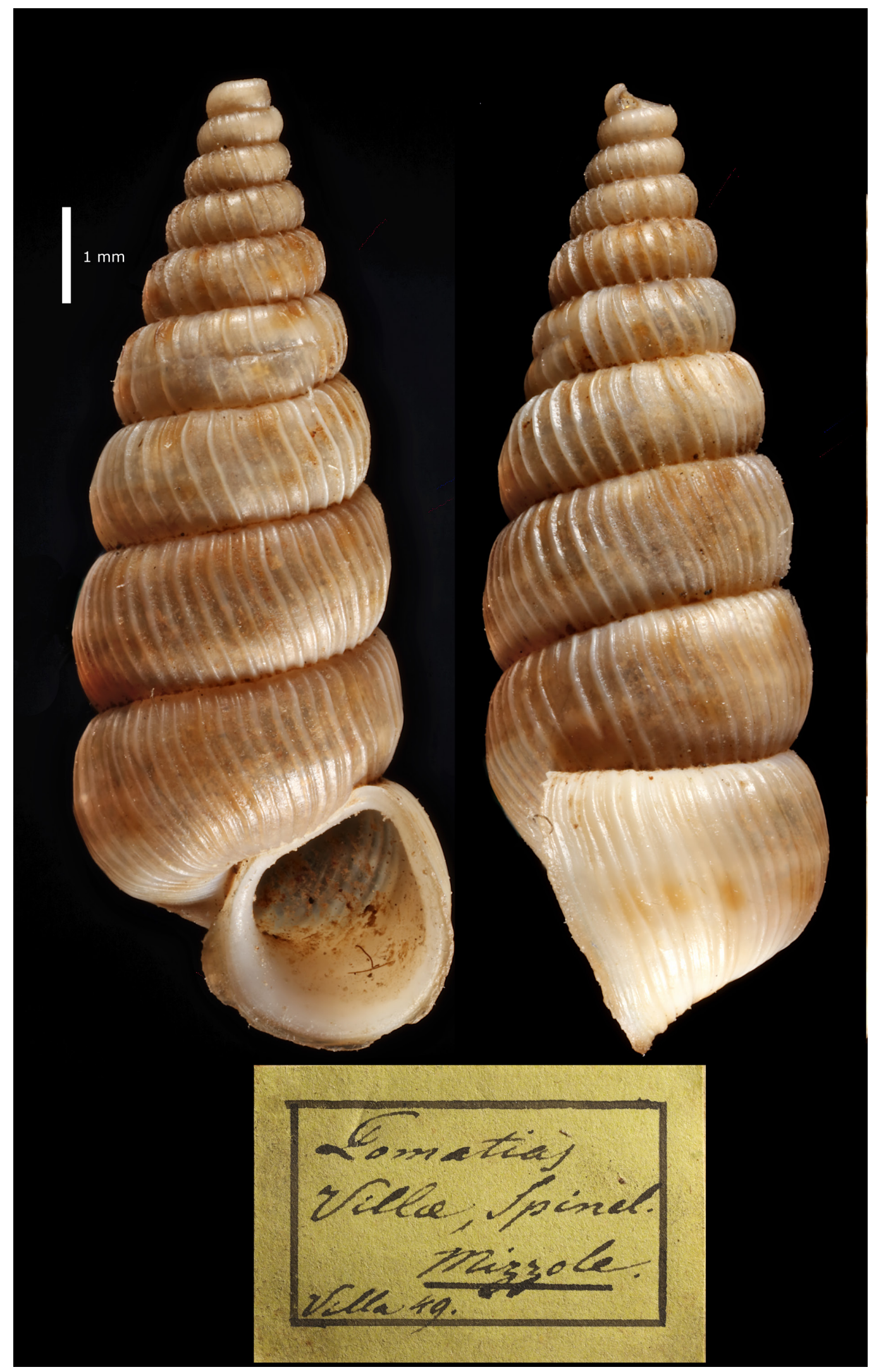

Fig. 19. Cochlostoma (Clessiniella) villae (Strobel, 1851), syntype, + , at the Museo di Storia Naturale of Parma University, Italy (PU1610-C1). 
esemplari raccolti a Mizzole presso Verona." = "P. villae Spinelli on specimens collected in Mizzole near Verona."

A few years later, De Betta \& Martinati (1855) gave almost the same poor description: "c. elongatior, costulis anfract. super. distinctioribus" = "taller shell, ribs in the upper whorls spaced" and follows "Prov. Verona, Mizzole. Spin. ex spec." = "Verona Province, Mizzole, specimens provided by Spinelli."

It could be, as was common at the time, that the specimens used to describe the species by both Strobel (1851) and De Betta \& Martinati (1855) have had a tortuous path, after being collected by Villa in 1849 (as it appears from Strobel's label), given to Spinelli later on, who gave the collector's name to the species (as far as is known, however, Giovanni Battista Spinelli never published the specific epithet

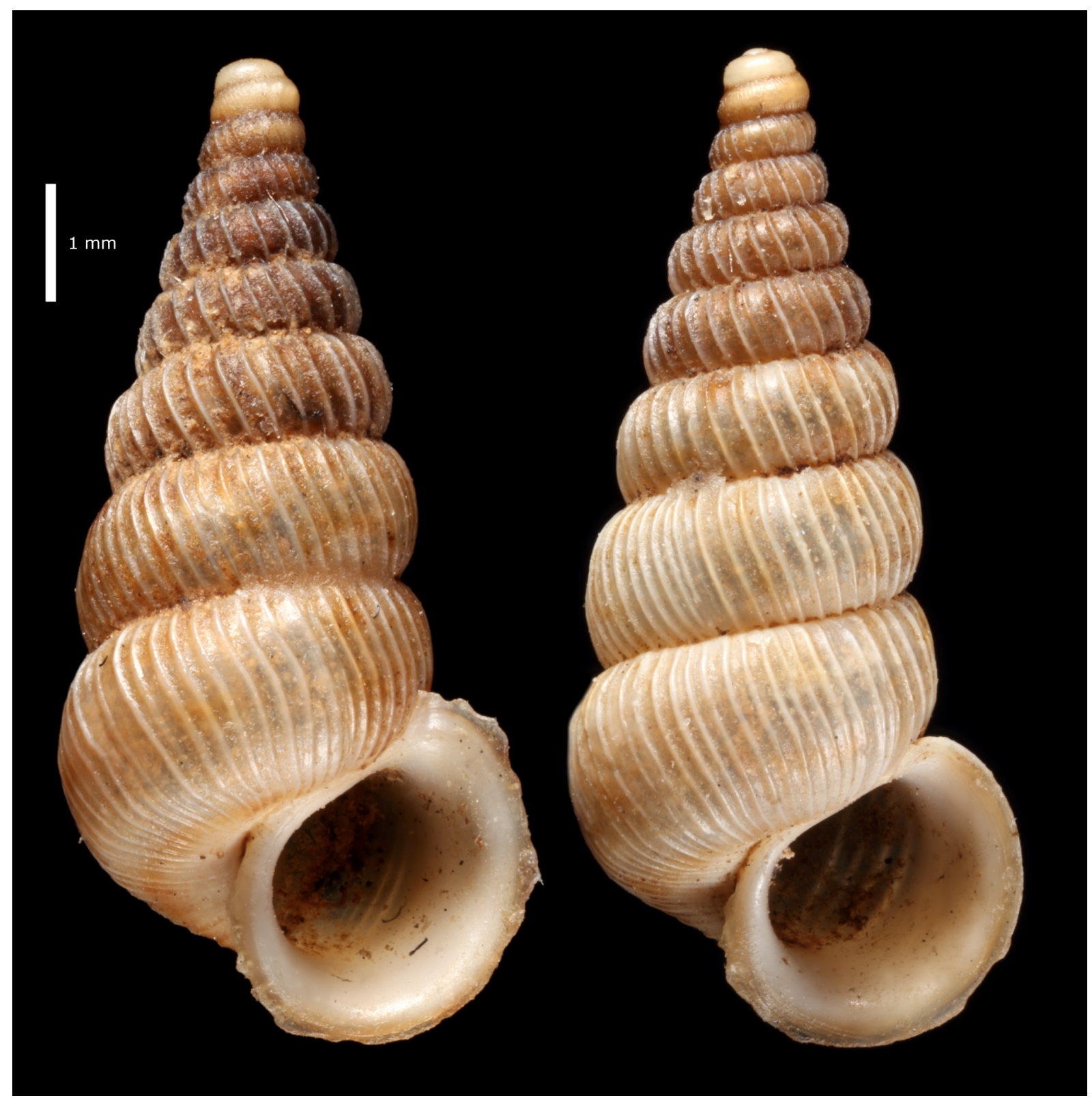

Fig. 20. Cochlostoma (Clessiniella) villae (Strobel, 1851), syntypes, $\widehat{\partial} \partial$, at the Museo di Storia Naturale of Parma University, Italy (1610-C2, left; 1610-C3, right). 


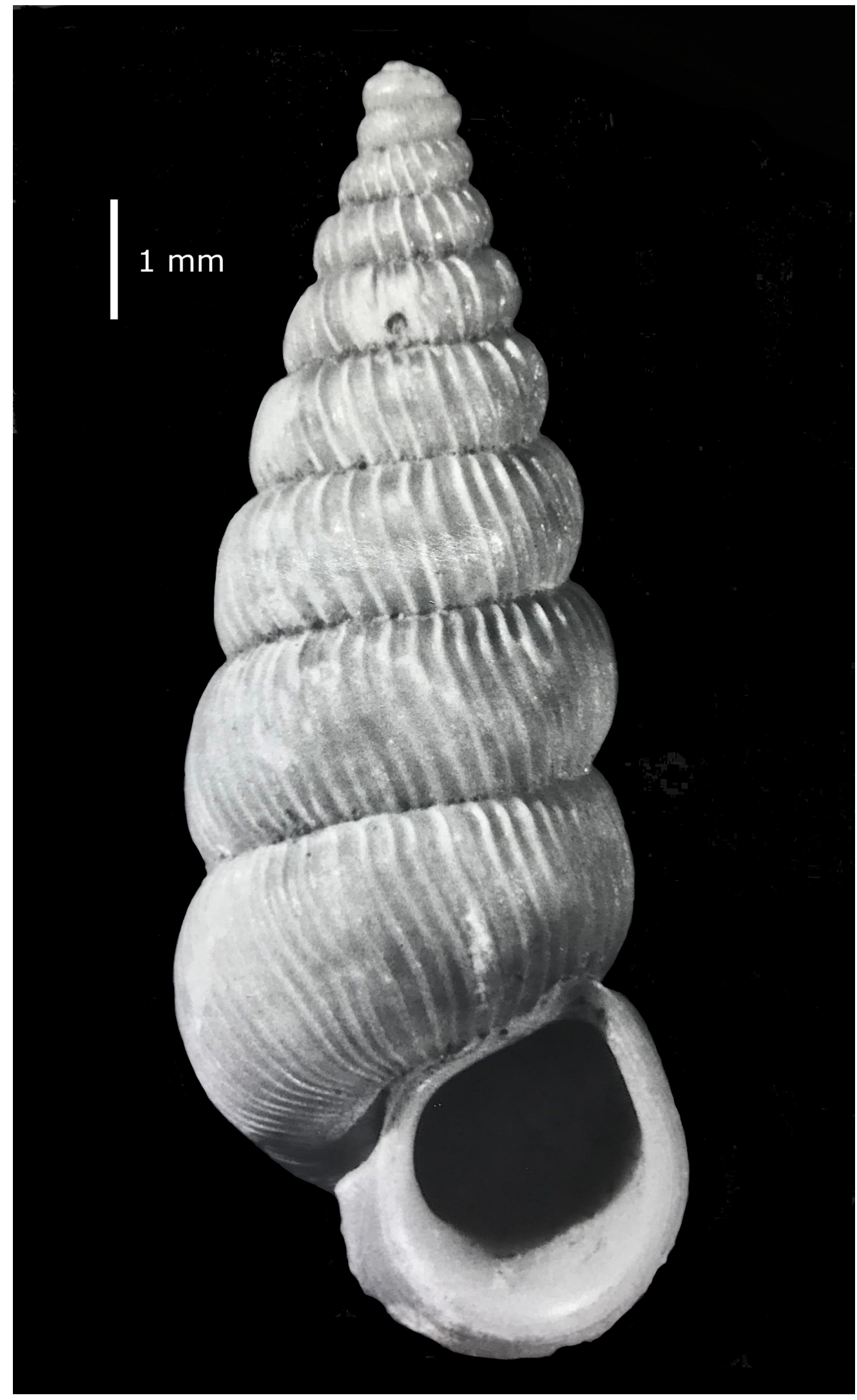

Fig. 21. Shell of Cochlostoma (Clessiniella) villae (Strobel, 1851). Collezione De Betta, Museo di Storia Naturale di Verona (photo Folco Giusti). 
villae, probably using the name only in private correspondence). Spinelli eventually gave part of the material to Strobel and part to De Betta (the De Betta material is currently kept at the Museo di Storia Naturale di Verona, see Fig. 21).

Cochlostoma (Clessiniella) anomphale Boeckel, 1939

Figs 1, 3A, 3A1, 14A, 15A, 16A, 22-24

Cochlostoma septemspirale anomphale Boeckel, 1939: 41-50 ("Karawanken, Trögern. Weg nach Vellach").

Cochlostoma septemspirale anomphale - Zilch 1958: 57.

Cochlostoma (Turritus) anomphale - Gittenberger 1971: 115-118.

Cochlostoma anomphale - Welter-Schultes 2012: 91.

\section{Diagnosis}

Shell quite densely ribbed, spots on the teleoconch generally clearly visible, body whorl inflated shortly before the lip. Visceral oviduct simplified with 1-2 superficial loops at most; seminal receptacle always club-shaped.

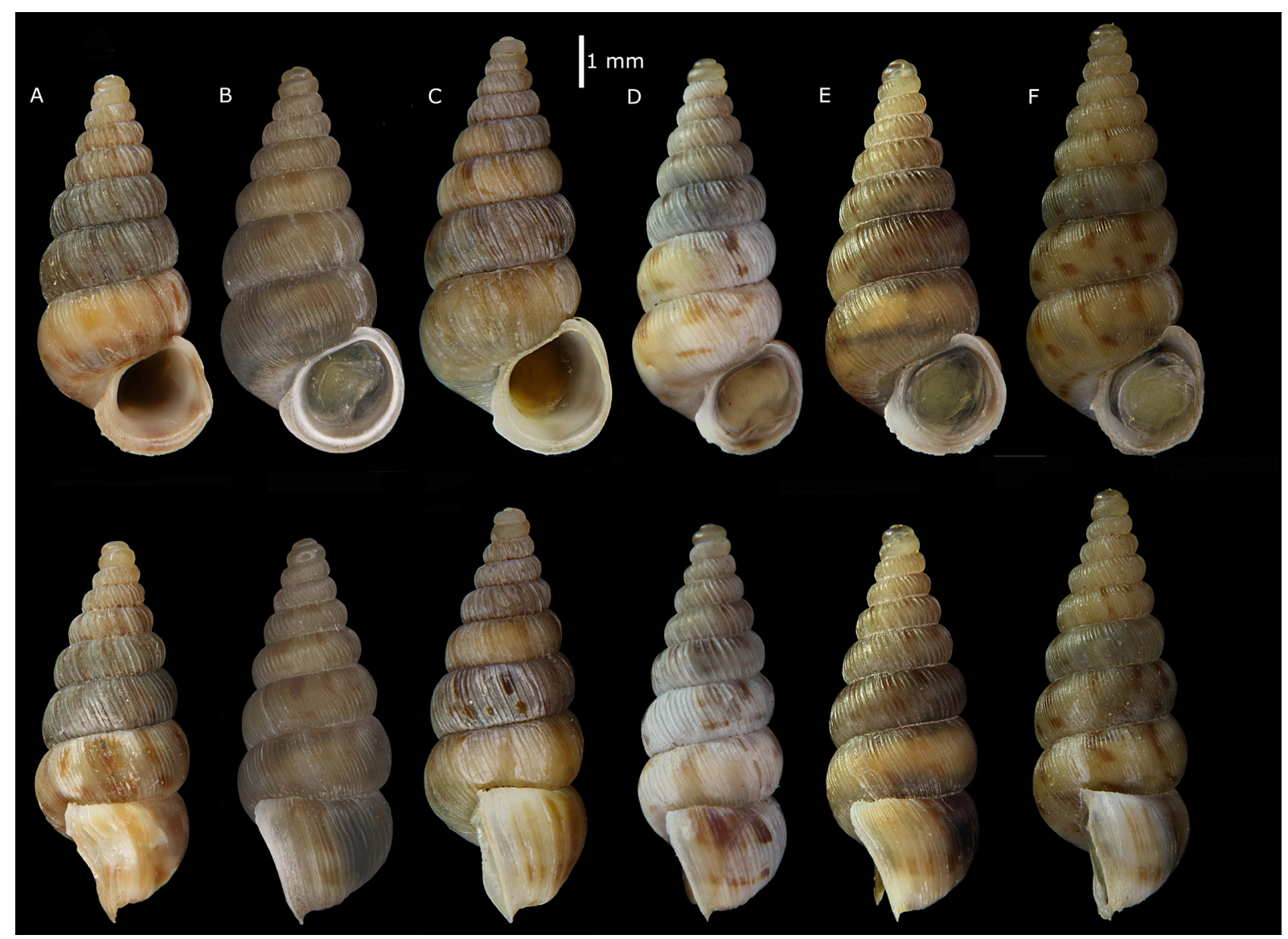

Fig. 22. Shell variability in Cochlostoma (Clessiniella) anomphale Boeckel, 1939. A. Savinjska Dolina, Slovenia (WdM1579). B. Eisenkappel, Austria (EZ0034). C. Stahovika, Slovenia (EZ0035). D. Vellach, Austria (EZ0991). E. Solčava, Slovenia (WdM7010). F. Logarska Dolina, Slovenia (EZ0994). 


\section{Type material}

\section{Paratypes}

AUSTRIA • 2 specs; Trögern, way to Vellach; SMF 42967/2 (Fig. 24).

Other material (we analyzed fresh material from the type locality)

AUSTRIA •EisenKappel; $46.48583^{\circ} \mathrm{N}, 14.59111^{\circ} \mathrm{E} ; 2010$, Mildner leg.; EZ0034 •Vellach; $46.42332^{\circ} \mathrm{N}$, $14.53639^{\circ}$ E; Aug. 2010; De Mattia leg.; EZ0991.

SLOVENIA • 210 road, N of Kokra; 46.34318 N, 14.48336 E; Aug. 2010; De Mattia leg.; EZ0993 - Gornji Grad; 46.2934 N, $14.82796^{\circ}$ E; Aug. 2010; De Mattia leg.; EZ0996 • Kamniska Bistrika; 46.32722 ${ }^{\circ}$ N, $14.59^{\circ}$ E; 2010; Zallot leg.; EZ0033 • Kokra; 46.30356 ${ }^{\circ}$ N, $14.4771^{\circ}$ E; Aug. 2010; De Mattia leg.; EZ0995 • Logarska Dolina; 46.41959 N, 14.65074 E; Aug. 2010; De Mattia leg.; EZ0994 - Logarska Dolina; 46.42334 N, 14.66301 E; Aug. 2010; De Mattia leg.; EZ0992 • PodvolovliekStahovica; 46.28787 ${ }^{\circ}$ N $14.69765^{\circ}$ E; Aug. 2005; Zallot leg.; EZ0036 • Savinjska Dolina; $46.36596^{\circ}$ N, $14.7318^{\circ}$ E; 2010; De Mattia leg.; WdM1579 - Solčava; $46.42002^{\circ} \mathrm{N}, 14.19172^{\circ}$ E; Jul. 2010; De Mattia leg.; WdM7010 • Stahovika; 46.26528 N, $14.60361^{\circ}$ E; 2005; Zallot leg.; EZ0035 • SV Anton; $46.28787^{\circ} \mathrm{N}, 14.69765^{\circ} \mathrm{E} ; 2010$; Zallot leg.; EZ0032.

\section{Remarks}

The holotype and 23 paratypes should be in the Boeckel collection at the Naturkundemuseum Erfurt (Hartmann et al. 2018: 26). However, the shells could not be traced when this article was prepared (C. Unger in mail October 14, 2020).Type locality - Austria, Trögern, way to Vellach.
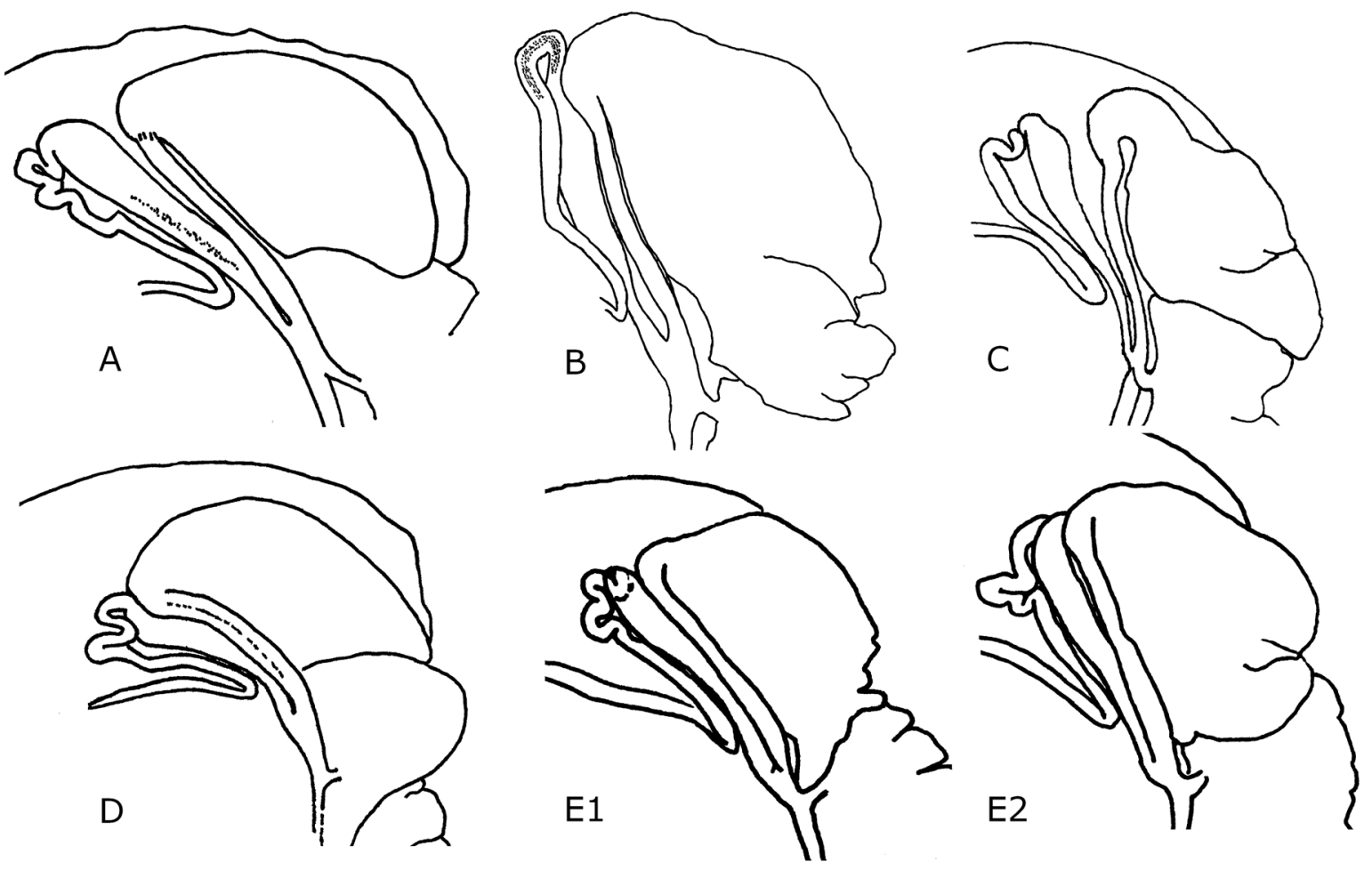

Fig. 23. Variation of the female genitalia in Cochlostoma (Clesssiniella) anomphale Boeckel, 1939. A. Eisenkappel, Austria (EZ0034). B. Savinjska Dolina, Slovenia (WdM1579). C. Vellach, Austria (EZ0991). D. Logarska Dolina, Slovenia (EZ0994). E1-E2. Solčava, Slovenia (WdM7010). 


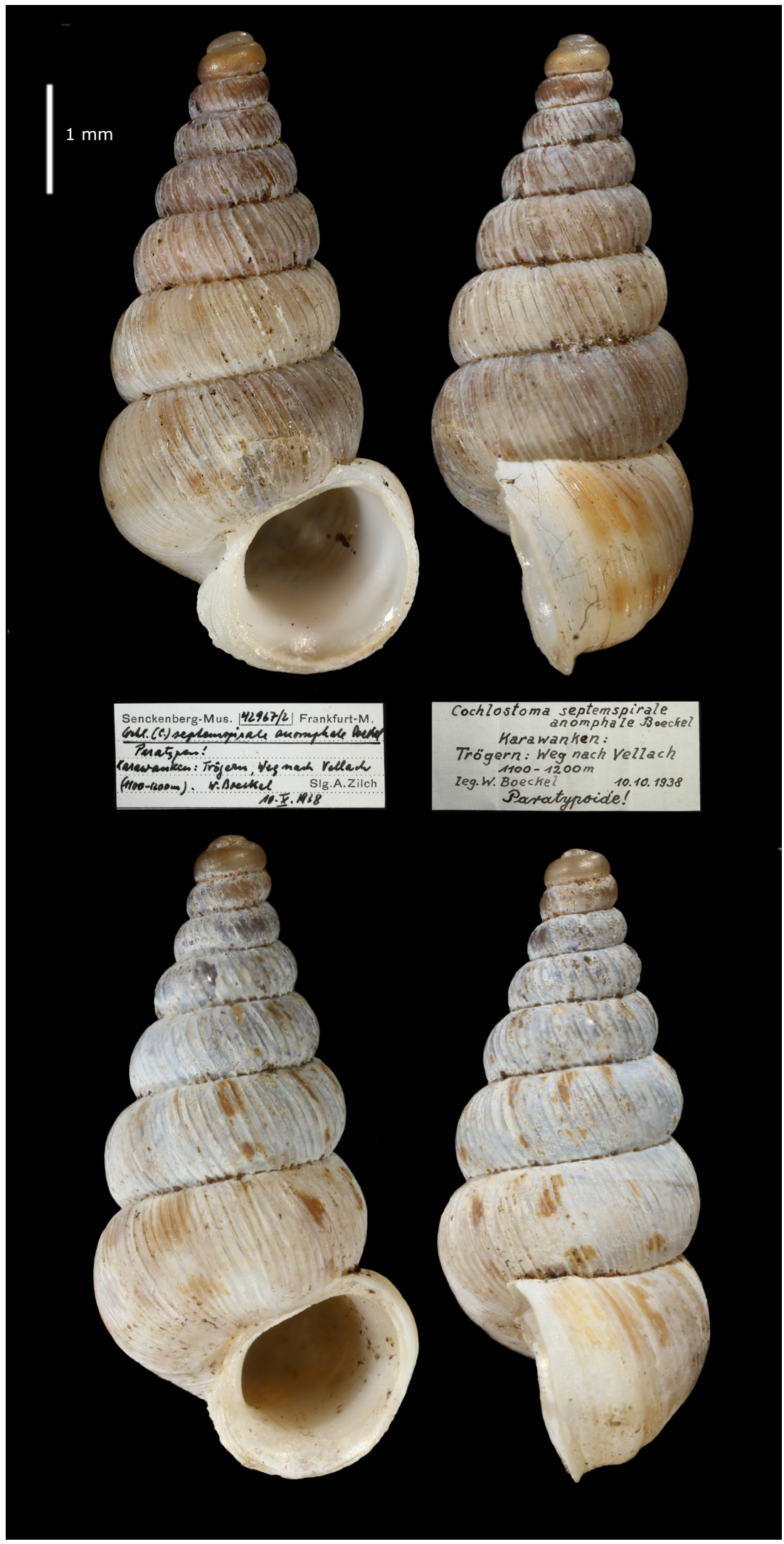

Fig. 24. Cochlostoma (Clessiniella) anomphale Boeckel, 1939. Paratypes at the Senckenberg Museum Frankfurt (SMF42967/2). 


\section{Description}

SHELL. The outside of the body whorl is inflated shortly before the apertural lip (Fig. 4A: OB). The lip is poorly developed; it is almost vertical at the palatal side, and curved backwards and covering the umbilicus at the columellar side. The shells are more densely sculptured and the ribs are weaker than in the other species of Clessiniella. Most of the shells have spots.

Female genitalia. There is a spindle-like seminal receptacle. The visceral oviduct has only 1-2, superficial, loops.

Male genitalia. The swollen penis is longer than the body and has a central spermiduct. There is a protruding, straight, body spermiduct.

\section{Distribution}

Cochlostoma (Clessiniella) anomphale inhabits the Karawanken in south-eastern Austria and northern Slovenia.

\section{Habitat preference}

The animals can be found under and on stones and boulders in shady habitats in forests.

\section{Remarks}

This species was initially described as a subspecies of Cochlostoma (Cochlostoma) septemspirale. Gittenberger (1971) considered it a separate species because of the sympatry with Co. (Co.) semptemspirale heydenianum and the lack of conchologically intermediate specimens. This is an epitome of quite common Cochlostoma taxonomical issues: based on shell morphology it was initially described as subspecies of a taxon which is only distantly related.

Cochlostoma (Clessiniella) stelucarum sp. nov. urn:1sid:zoobank.org:act:4609B41C-3611-4F8A-9048-5F97EA0446E6

Figs 1, 3E, 14B, 15B, 16B, 15-27

\section{Diagnosis}

Slender shell with $8 \frac{1}{2}-10^{1 / 2}$ rather widely ribbed whorls. Spots always visible. Poorly developed lip with FF or AF external lobe. Visceral oviduct simplified and seminal receptacle sometimes oval.

\section{Etymology}

The epithet 'steluca' is an acronym noun formed from the initial syllables of the names of the wife and two daughters (Stefania, Ludovica and Camilla Zallot) of the senior author.

Type material (Fig. 27)

\section{Holotype}

ITALY • + (in ethanol); Friuli Venezia Giulia, Udine, Cornino; $46.2297^{\circ} \mathrm{N}, 13.0250^{\circ} \mathrm{E} ; 180 \mathrm{~m}$ a.s.l.; 5 Jun. 2002; Zallot leg.; rocks surrounding the lake; RMNH.MOL.347617.

\section{Paratypes}

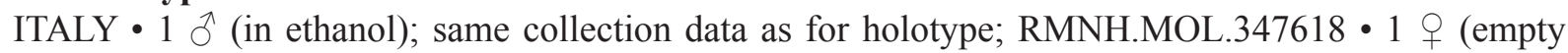
shell); same collection data as for holotype; GenBank voucher H3: KX120830; RMNH.MOL.347619. 


\section{Other material}

ITALY • Anduins-cliffs; $46.23755^{\circ} \mathrm{N}, 12.97106^{\circ} \mathrm{E}$; 1997 ; Zallot leg.; EZ0102 • Campone; $46.26194^{\circ} \mathrm{N}$, $12.83333^{\circ} \mathrm{E}$; 2010; Zallot leg.; EZ0098 • Castelmonte; $46.094^{\circ} \mathrm{N}, 13.52095^{\circ} \mathrm{E}$; Oct. 2010; Margelli leg.; EZ1020 • Cjanet-Peonis; $46.25917^{\circ} \mathrm{N}, 13.04111^{\circ} \mathrm{E}$; 2002; Zallot leg.; EZ0105 • Cornino; $46.22972^{\circ} \mathrm{N}$, 13.025 E; 2002; Zallot leg.; EZ0104 • F. Piccola Mt Valinins; $46.245^{\circ} \mathrm{N}, 12.80111^{\circ}$ E; 2000; Zallot leg.; EZ0095 • Mt Bernadia; 46.23361 ${ }^{\circ} \mathrm{N}, 13.25556^{\circ} \mathrm{E}$; 2005; Zallot leg.; EZ0107 • Mt Ciaurlec; 46.21226 ${ }^{\circ} \mathrm{N}, 12.88018^{\circ}$ E; 2005; Zallot leg.; EZ0099 • Mt Prat; $46.24577^{\circ} \mathrm{N}, 12.9907^{\circ}$ E; 2002; Zallot leg.; EZ0103 • Mt Valinis; 46.23417 ${ }^{\circ}$ N, $12.8075^{\circ}$ E; 2002; Zallot leg.; EZ0096 • San Vito D'asio; $46.2337^{\circ} \mathrm{N}, 12.94531^{\circ} \mathrm{E}$; 1997 ; Zallot leg.; EZ0101 • Tramonti-Campone; $46.27833^{\circ} \mathrm{N}, 12.81917^{\circ} \mathrm{E}$; 2000; Zallot leg.; EZ0097 • Val di Torre-all'imbocco; 46.23861 ${ }^{\circ} \mathrm{N}, 13.23111^{\circ} \mathrm{E}$; 1998; Zallot leg.; EZ0106 • Valle D’Arzino; 46.30278 N, $12.92972^{\circ}$ E; 2000; Zallot leg.; EZ0100.

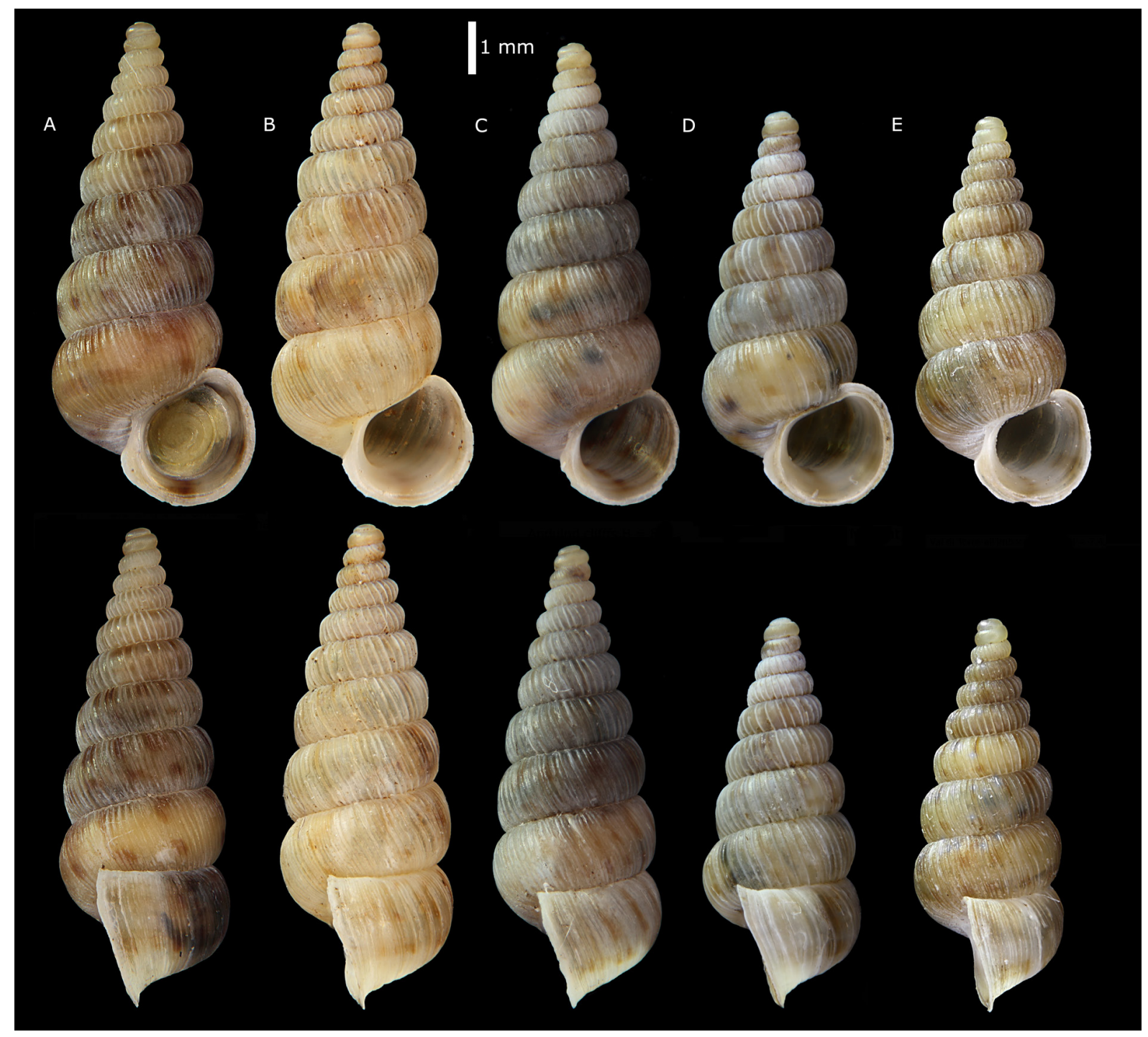

Fig 25. Shell variability in Cochlostoma (Clessiniella) stelucarum sp. nov. A. Cornino, Italy (EZ0104). B. Mt Valinis, Italy (EZ0096). C. Anduins, Italy (EZ0102). D. Mt Prat, Italy (EZ0103). E. Val di Torre, Italy (EZ0106). 


\section{Description}

SHELl. The shell has prominent spots and strong and rounded ribs, which are regularly and widely spaced and coloured like the background. The ribs become less prominent on the body whorl. The body whorl slightly and gradually enlarges while approaching the aperture (Fig. 4A: FF, AF). In comparison with the other species of the subgenus, the whorls increase more slowly in size so that, given the same shell height, there is a higher number of whorls (Fig. 9).

Female GENitalia. The visceral oviduct has only 1-2 superficial loops, what makes it one of the simplest in the genus Cochlostoma. The seminal receptacle is thin and may be oval, thus not always club-shaped as in the other species.

MaLE genitalia. The penis is longer than the body and inflated, although less so than in the other species of Clessiniella. The penial spermiduct is gently twisted. The body spermiduct is straight. There is a long groove, ending in a well-formed sperm pocket, delimited at the frontal side by an ascending sperm funnel.

\section{Distribution}

The species inhabits a restricted area in the eastern Southern Pre-Alps. Our sampling localities are located between Val Tramontina to the west and Val di Torre to the east (Friuli region, Italy).

\section{Habitat preference}

Like in most species of Cochlostoma, these are obligate rock-dwelling snails but, rather than on the surface of limestone cliffs, their main habitat is on smaller surfaces of stones and boulders which are normally found in large numbers at the foot of the cliffs.
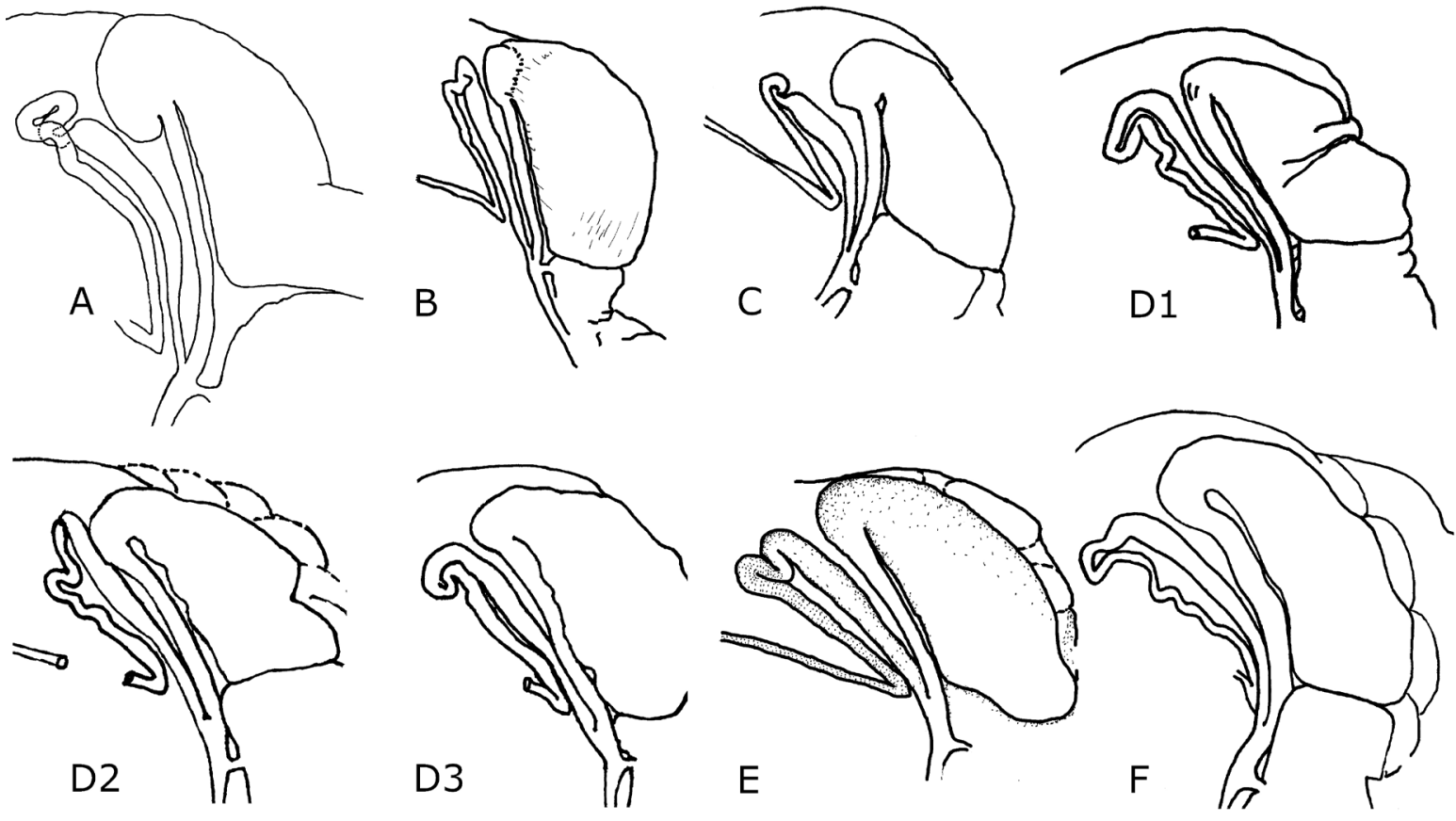

Fig 26. Variation of the female genitalia in Cochlostoma (Clessiniella) stelucarum sp. nov. A. Mt Valinis, Italy (EZ0096). B. Campone, Italy (EZ0098). C. Anduins, Italy (EZ0102). D1-D3. Cornino, Italy (EZ0104). E. Cjanet-Peonis, Italy (EZ0105). F. Mt Bernadia, Italy (EZ0107). 


\section{Remarks}

Cochlostoma (Clessiniella) stelucarum sp. nov. was introduced as Cochlostoma sp. by Zallot (2002: 98). At most of the localities, it lives syntopically with either Co. (Eupomatias) philippianum (Gredler, 1853) or Co. (Eupomatias) henricae (Strobel, 1851). Near the village of Anduins it has been found a few centimeters apart from both Co. (Eu.) henricae and Co. (Cochlostoma) septemspirale on stones and boulders. This is one of the rare cases where 3 species of Cochlostoma, belonging to different subgenera, live syntopically. The range of the species is situated in between the range of $\mathrm{Co}$. $(\mathrm{Cl}$.) villae in the west and that of the other species of Clessiniella in the east.
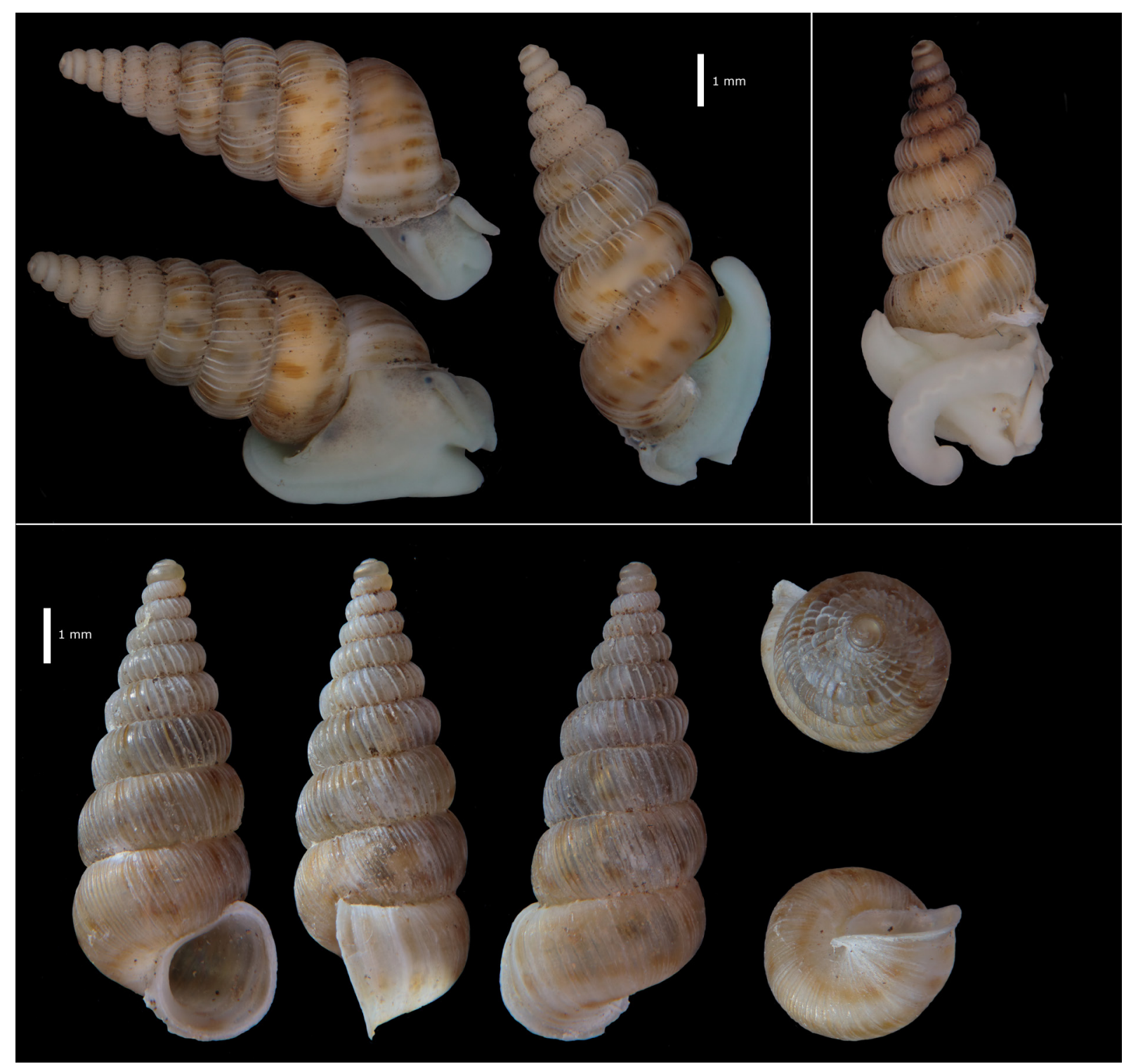

Fig. 27. Cochlostoma (Clessiniella) stelucarum sp. nov. Top left: holotype, $q$ (RMNH.MOL.347617), $\mathrm{H}=8 \mathrm{~mm}$. Top right: paratype, $\widehat{\jmath}$ (RMNH.MOL.347618), $\mathrm{H}=7 \mathrm{~mm}$. Bottom: paratype, $\varnothing$ (RMNH. MOL.347619), empty shell, $\mathrm{H}=8.7 \mathrm{~mm}$. 
Cochlostoma (Clessiniella) tergestinum (Westerlund, 1878)

Figs 1, 3B, 5, 14C, 15C, 16C, 28-30, 34 (bottom), 35 (bottom), 36 (top)

Pomatias tergestinus Westerlund, 1878: 108-109 (“Triest”).

Pomatias tortivus Westerlund, 1885: 124 (“Fiume, San Daniel u. Karstgebirge”, which should be Rijeka in Croatia and Štanjel in Slovenia).

Pomatias tergestinus var. grahovana A.J. Wagner, 1897: 616, pl. 9 fig. 89 (“Grahovo bei Fiume”, a locality ca $10 \mathrm{~km}$ north of Rijeka in Croatia).

Pomatias tergestinus var. tortiva - A.J. Wagner 1897: 616, pl. 9 fig. 88.

Cochlostoma tergestinum - Welter-Schultes 2012: 102.

\section{Diagnosis}

Shell very variable, lip curved backwards more or less abruptly at the columellar side. Strong ribs on the teleoconch, in most of the populations widely spaced and, on the upper whorls, whitish and contrasting with the corneous, dark brown to light yellow, shell background. Visceral oviduct with convoluted loops path; body spermiduct straight.

\section{Type material}

\section{Syntypes}

ITALY • "Triest" [Friuli Venezia Giulia, Trieste]; Westerlund leg.; SMF160677/1 • same label data as for preceding; SMF 160676/7 (Fig. 30).

\section{Other material}

AUSTRIA - Dobratsch; 46.59222 ${ }^{\circ}$ N, $13.6825^{\circ}$ E; 2009; Mildner and Giovannelli leg.; EZ0799 • Kranzwand; $46.588799^{\circ}$ N, $13.75002^{\circ}$ E; Jul. 2015; AL leg.; NHMW109000.

CROATIA • Sveta Nedelja near Labin; $45.13741^{\circ} \mathrm{N}, 14.14488^{\circ}$ E; 2000; De Mattia leg.; WdM6961 - Brest, Cicaria; $45.45444^{\circ} \mathrm{N}, 14.00625^{\circ} \mathrm{E}$; Apr. 2008; De Mattia leg.; WdM6715 • Drivenik spring; $45.24147^{\circ} \mathrm{N}, 14.64828^{\circ} \mathrm{E}$; Mar. 2009; De Mattia leg.; WdM6714 • from A6 to 501 down Bakar; $45.30543^{\circ} \mathrm{N}, 14.58579^{\circ} \mathrm{E}$; Jul. 2009; De Mattia and Zallot leg.; EZ0938 - Jakačici; $45.179494^{\circ} \mathrm{N}$, $14.004384^{\circ}$ E; 2009; De Mattia leg.; WdM6699 - Kremenje-Buje; $45.43558^{\circ} \mathrm{N}$, $13.68499^{\circ} \mathrm{E}$; Jan. 2008; De Mattia leg.; WdM6400 • Kukurini; $45.19369^{\circ}$ N, 14.07216 E; Feb. 2009; De Mattia leg.; WdM6698 • Limski Kanal; 45.12957 ${ }^{\circ}$ N, $13.74039^{\circ}$ E; 2010; De Mattia leg.; WdM6693 • Limski Kanal; $45.12957^{\circ}$ N, $13.74039^{\circ}$ E; Jun. 2010; De Mattia leg.; WdM6998 • Mala Ucka; $45.27605^{\circ} \mathrm{N}$, 14.19715 E; 2009; De Mattia leg.; WdM6768 • Meja, Bakarac; $45.3037^{\circ} \mathrm{N}, 14.58348^{\circ}$ E; 2009; De Mattia leg.; WdM6951 • Momjan; 45.442 ${ }^{\circ}$ N, $13.71384^{\circ}$ E; 2009; De Mattia leg.; WdM6854 • Mt Učka, beech forest; $45.30636^{\circ} \mathrm{N}, 14.20865^{\circ} \mathrm{E}$; 2009; De Mattia leg.; WdM6794 • Mt Učka, top; $45.28967^{\circ} \mathrm{N}$, 14.20341 ${ }^{\circ}$ E; 2009; De Mattia leg.; WdM6794 • Pazin; $45.24134^{\circ}$ N, $13.93099^{\circ}$ E; Jun. 1986; Drimmer leg.; HNHM97227 • Permani; $45.43742^{\circ} \mathrm{N}, 14.28905^{\circ}$ E; 2009; De Mattia leg.; WdM6708 • Raša; $45.0872^{\circ} \mathrm{N}, 14.08854^{\circ} \mathrm{E} ; 2010$; De Mattia leg.; WdM6968 • Rasko Polje; $45.15638^{\circ} \mathrm{N}, 14.0538^{\circ} \mathrm{E}$; 2010; De Mattia leg. EZ0813 • Rasko Polje; $45.15638^{\circ}$ N, $14.0538^{\circ}$ E; 1900; De Mattia leg.; EZ1027

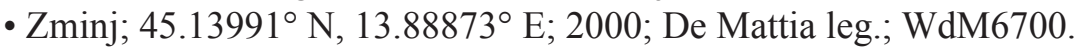

ITALY • Val Rosandra; 45.62 ${ }^{\circ}$ N, $13.87139^{\circ}$ E; 2000; Zallot leg.; EZ0840 • Torri di Monrupino; $45.71878^{\circ} \mathrm{N}, 13.80594^{\circ} \mathrm{E}$; 1998; Zallot leg.; EZ0839.

SLOVENIA - Abram-Nanos Mt; 45.82055 N, $14.01433^{\circ}$ E; 2008; De Mattia leg.; WdM6731 - Brestovica pri Komnu; 45.80996 ${ }^{\circ} \mathrm{N}, 13.65016^{\circ} \mathrm{E} ; 2009$; De Mattia leg.; WdM6948 - Čepno; $45.67071^{\circ} \mathrm{N}, 14.09732^{\circ}$ E; Jun. 2009; De Mattia leg.; WdM6866 • Črnotiče-Črni Kal; $45.55276^{\circ} \mathrm{N}$, 
13.88633 ${ }^{\circ}$ E; Mar. 2009; De Mattia leg.; EZ1028 - Dolina Nanos-Abran-Podkraj; $45.79813^{\circ} \mathrm{N}$, $14.06699^{\circ}$ E; Apr. 2009; De Mattia leg.; WdM6733 • Farjev Vrh; $45.92429^{\circ}$ N, $13.95442^{\circ}$ E; 2009; De Mattia leg.; WdM6740 • Golac, Vela Vrata; 45.51315 N, 14.02969 E; 2009; De Mattia leg.; WdM6762 - Gorjansko; 45.78485 ${ }^{\circ}$ N, $13.70068^{\circ}$ E; 2000; De Mattia leg.; WdM6950 • Hruščica; $45.85832^{\circ} \mathrm{N}$, 14.1117 E; 2000; De Mattia leg.; WdM2409 • Ilirska Bistrika; 45.56944 N, $14.23917^{\circ}$ E; 2009;

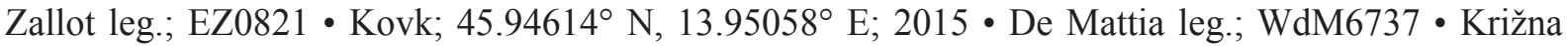
Jama; $45.82003^{\circ} \mathrm{N}, 14.24579^{\circ}$ E; Jun. 2009; Jochum leg.; HNHM97142• Markovščina-Dimnice cave; $45.58357^{\circ} \mathrm{N}, 14.03994^{\circ} \mathrm{E}$; 2009; De Mattia leg.; WdM6765 • Mt Kobilica; $46.06528^{\circ} \mathrm{N}, 13.80907^{\circ} \mathrm{E}$; 2005; De Mattia leg.; WdM6849 • Mt Nanos, top, loc. Plesa; $45.79813^{\circ} \mathrm{N}, 14.06699^{\circ} \mathrm{E}$; Jun. 2005; De Mattia leg.; WdM0000 • Mt Snežnik 1161; 45.56816 N, $14.37049^{\circ}$ E; Jun. 2010; De Mattia leg.; WdM7012 • Mt Snežnik 1390; 45.57179 N, 14.41044 E; Jun. 2010; De Mattia leg.; EZ0965 •

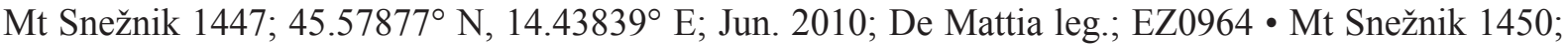
45.58195 ${ }^{\circ}$ N, $14.43129^{\circ}$ E; Jun. 2010; De Mattia leg.; EZ0962 • Mt Snežnik car parking; $45.58235^{\circ} \mathrm{N}$, $14.43117^{\circ}$ E; Jun. 2002; De Mattia leg.; WdM7006 • Mt Vremščica; $45.69898^{\circ}$ N, $14.03388^{\circ}$ E; 2009;

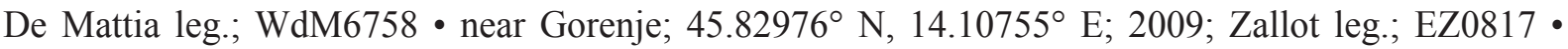
near Logatec; $45.88076^{\circ} \mathrm{N}, 14.27448^{\circ} \mathrm{E}$; 2009; Zallot leg.; EZ0828 • Osp, $45.57071^{\circ} \mathrm{N}, 13.86168^{\circ} \mathrm{E}$; 2010; De Mattia leg.; WdM6756 • Ospo, small cliff; $45.57119^{\circ} \mathrm{N}, 13.8582^{\circ} \mathrm{E}$; May 2010; De Mattia leg.; WdM6993 • Otlica; 45.92822 ${ }^{\circ}$ N, $13.90918^{\circ}$ E; 1986; De Mattia leg.; WdM6752 • Planina; $45.82405^{\circ} \mathrm{N}, 14.24708^{\circ} \mathrm{E}$; 2000; Zallot leg.; EZ0823 • Planina; $45.82972^{\circ} \mathrm{N}, 14.25056^{\circ} \mathrm{E}$; 2000; Zallot leg.; EZ0824・ Planina, Postojna; $45.83^{\circ} \mathrm{N}, 14.26056^{\circ}$ E; 2009; Zallot leg.; EZ0826・ Podgorje; $45.53872^{\circ} \mathrm{N}, 13.9449^{\circ} \mathrm{E}$; 2000; De Mattia leg.; WdM6735 • Podgorje; $45.5364^{\circ} \mathrm{N}, 1^{\circ} .9463^{\circ} \mathrm{E} ; 2009$; De Mattia leg.; WdM6735 • Podlešče-Bainsizza; 46.05738 ${ }^{\circ}$ N, $13.72709^{\circ}$ E; May 2009; De Mattia leg.; WdM6814 • Prešnica; 45.56587 N, $13.94191^{\circ}$ E; Mar 2009; De Mattia leg.; WdM6734 • Pri CerkviBainsizza; $46.04133^{\circ} \mathrm{N}, 13.77584^{\circ} \mathrm{E}$; 2009; De Mattia leg.; WdM6834 • Rakow Skocjan; $45.79467^{\circ} \mathrm{N}$, 14.28948 ${ }^{\circ}$ E; 2010; Zallot leg.; EZ0838 • San Canzian (Škocjan); $45.66532^{\circ} \mathrm{N}, 13.99356^{\circ}$ E; 2009; De Mattia leg.; WdM6754 • Senožeče; $45.71421^{\circ} \mathrm{N}, 14.07856^{\circ} \mathrm{E} ; 2009$; De Mattia leg.; WdM6865 • $10 \mathrm{~km}$ S of Vrhnika; $45.88549^{\circ} \mathrm{N}, 14.26091^{\circ} \mathrm{E}$; 2000; Zallot leg.; EZ0827 •Vrhnika, Močilnik; $45.95591^{\circ} \mathrm{N}$, 14.29268 E; 2009; Zallot leg.; EZ0829.

\section{Remarks}

The original indication of the type locality is rather vague, since only a city in the Friuli-Venezia Giulia region, in the easternmost part of Italy is indicated. The closest to Trieste where we found the species is at "Val Rosandra", $8 \mathrm{~km}$ north-east of the city. The fresh specimens collected there have shell features similar to those of the syntypes. We therefore consider them as topotypical.

\section{Description}

Shell. The size of the shells is extremely variable (height $6.1-9.6 \mathrm{~mm}$ in the females) as well as their general morphology (see Fig. 28). Several populations are spotless whereas others have clear spots on the teleoconch. Depending on the population, the lip can be either poorly or even hugely developed. In lateral view, the body whorl, while approaching the aperture, may widen in various ways, depending on the population (Fig. 4A: FF, AF, II). The H/W ratio is very variable and the slenderness of the shells in different populations varies accordingly.

Female genitalia. There is a long, club-shaped seminal receptacle. In some cases there is a change of colour at its base, presumably indicating the starting point of the rather long distal oviduct. As shown in Table 5, there is a considerable variability in the part of the seminal receptacle that is curved down and in the number of loops.

Male genitalia. The inflated penis is longer than the body. The penial spermiduct is confined to the central part of the penis and gently twisted. The body spermiduct is straight and well defined. 


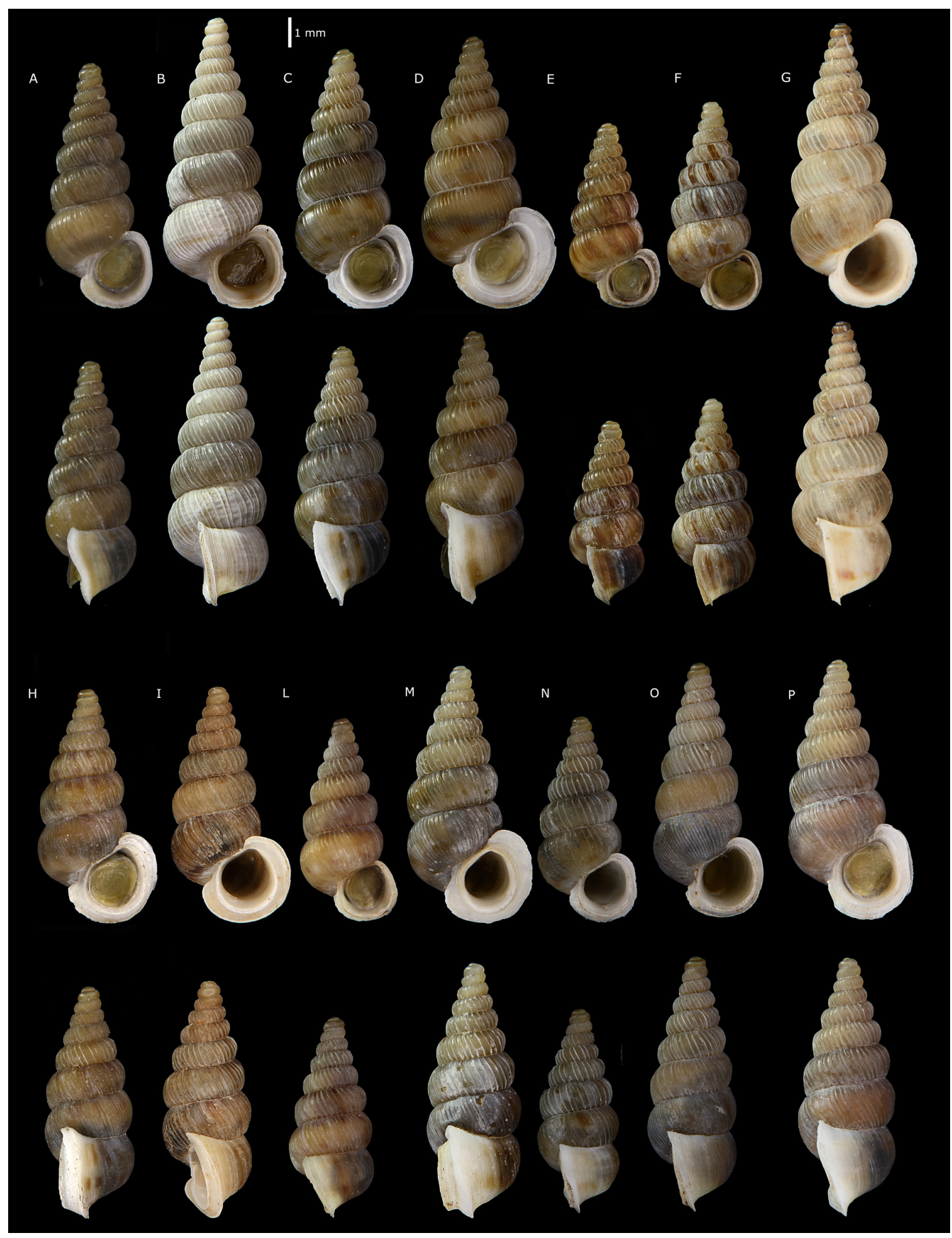

Fig. 28. Shell variability in Cochlostoma (Clessiniella) tergestinum (Westerlund, 1878). A. Mt Kobilica, Slovenia (WdM6849). B. Limski Kanal, Croatia (WdM6693). C. Dobratsch, Austria (EZ0799). D. Farjev Vrh, Slovenia (WdM6740). E. Rasko Polje, Croatia (EZ0813). F. Raša, Croatia (WdM6968). G. Val Rosandra, Italy (EZ840). H. Mala Učka, top, Croatia (WdM6794). I. Ilirska Bistrika, Slovenia (EZ0921). L. Permani, Croatia (WdM6708). M. Vrhnika, Slovenia (EZ0827). N. Near Bakar, Croatia (EZ0938). O. Mala Učka, Croatia (WdM6768). P. Mt Snežnik 1161, Slovenia (WdM7012). 


\section{Distribution}

This species inhabits the eastern side of the province of Trieste in Italy, the southwestern part of Slovenia and the Istrian peninsula. It has also been found in several Croatian localities in the surroundings of Bakar and, quite far from its main range, on the Villacher Alps, in Austria (see Mildner 1997). The species has been erroneously reported for Albania (see Fehér \& Erőss 2009).

\section{Habitat preference}

It is an obligate rock-dwelling species. Co. $(\mathrm{Cl}$.) tergestinum is found in a variety of habitats, from open, exposed limestone cliffs to shady spots in deciduous and evergreen forests.

\section{Remarks}

According to Zilch (1958) and Alzona (1971) there are 3 subspecies of Co. (Cl.) tergestinum, viz. the nominate subspecies Co. (Clessiniella) tergestinum tergestinum (Westerlund, 1878), Co. (Clessiniella) tergestinum grahovanum (A.J. Wagner, 1897) and Co. (Clessiniella) tergestinum tortivum (Westerlund, 1885).
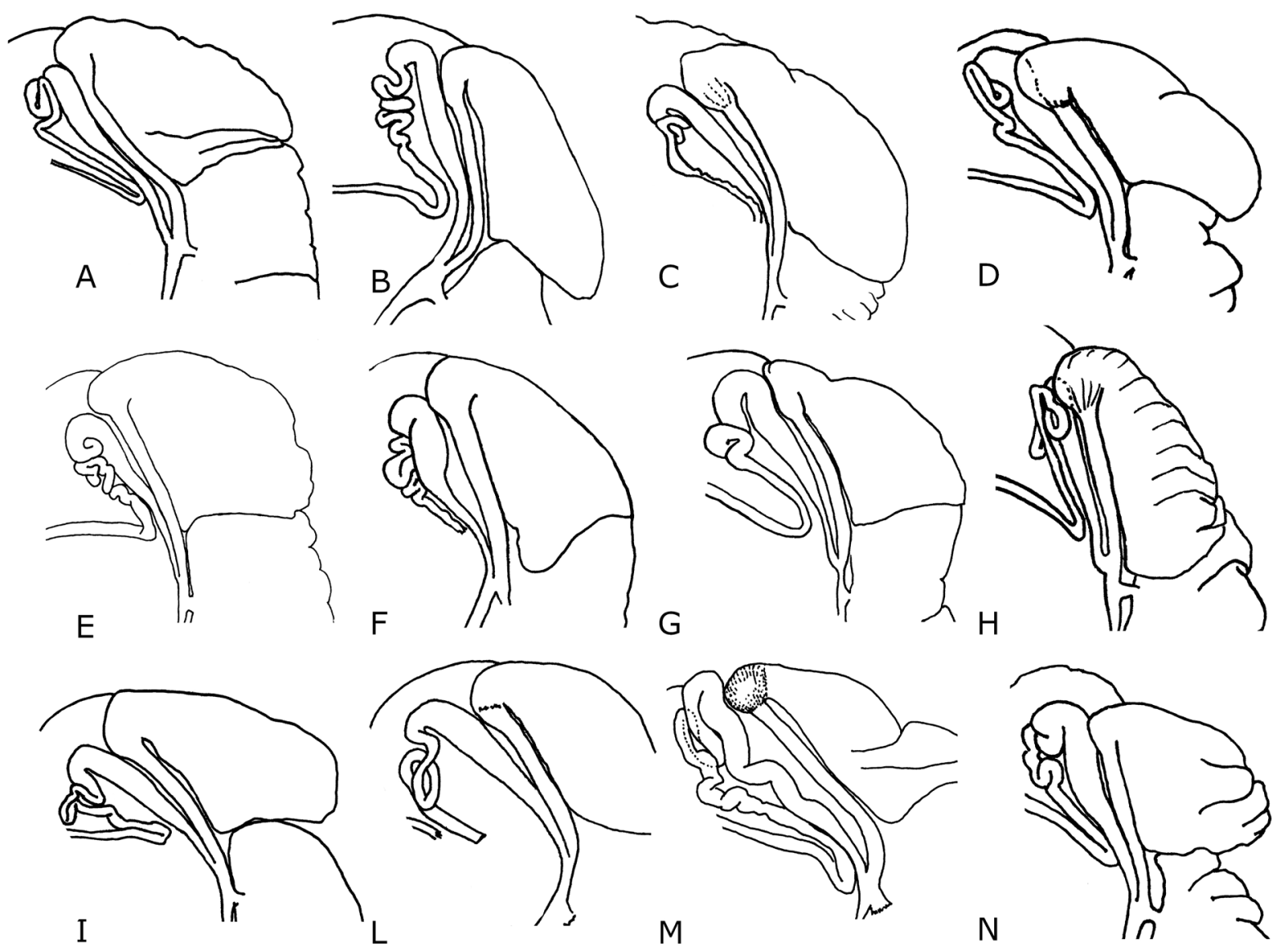

Fig. 29. Variation of the female genitalia in Cochlostoma (Clessiniella) tergestinum (Westerlund, 1878). A. Pri Cerkvi, Slovenia (WdM6834). B. Brestovica, Slovenia (WdM6948). C. Dobratsch, Austria (EZ0799). D. Goriansko, Slovenia (WdM6950). E. Prešnica, Slovenia (WdM6734). F. Farjev Vrh, Slovenia (WdM6740). G. Rasko Polje, Croatia (EZ0813). H. Rasa, Croatia (WdM6968). I. Permani, Croatia (WdM6708). L. Meja-Bakarac, Croatia (WdM6951). M. Jakačici, Croatia (WdM6699). N. Drivenik, Croatia (WdM6714). 


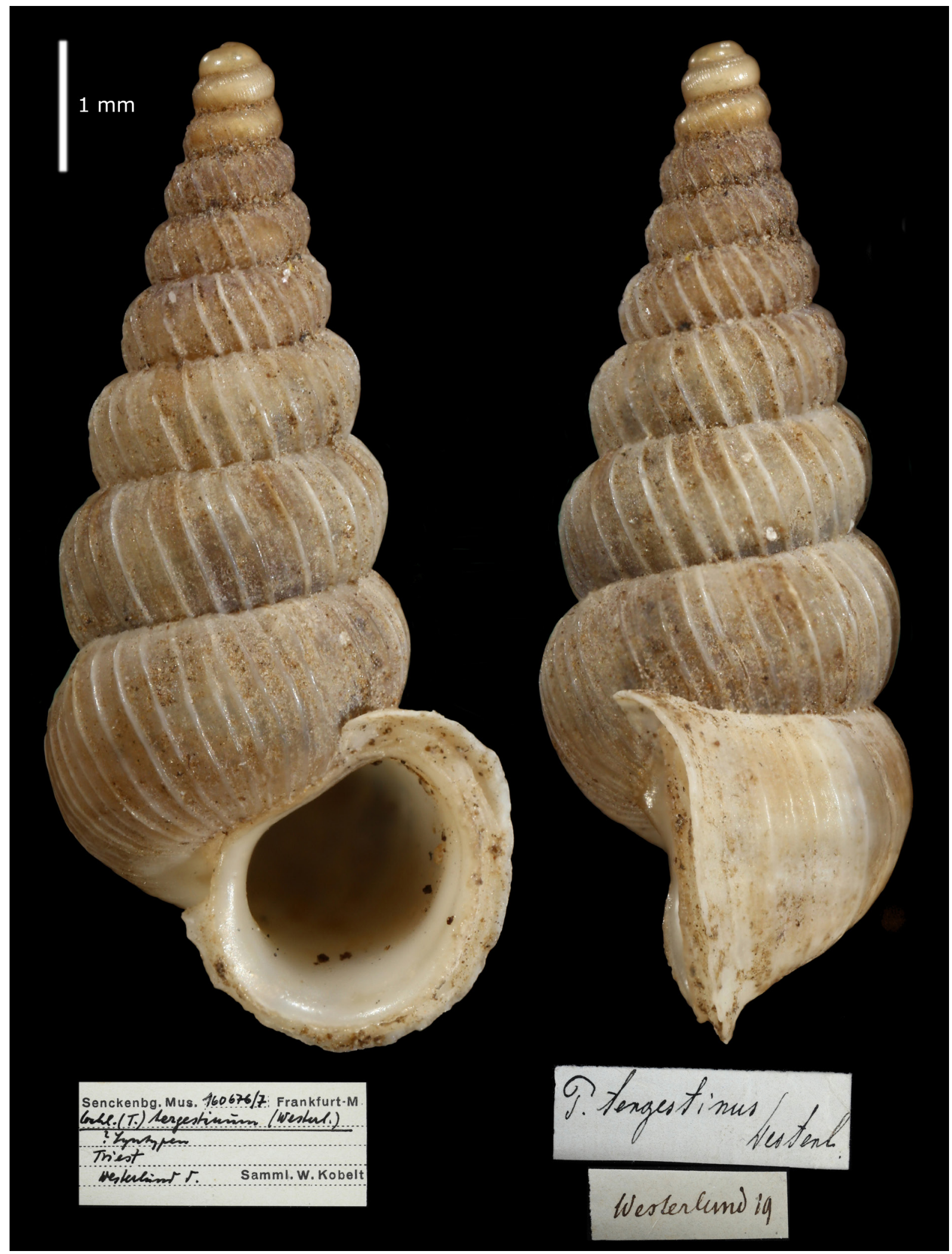

Fig. 30. Cochlostoma (Clessiniella) tergestinum (Westerlund, 1878), syntype at the Senckenberg Museum Frankfurt (SMF160676/7). 
For the time being, we here synonymize these nominal taxa because the conchological features which supposedly characterize them, like measures, density and prominence of the ribs, and the structure of the lip, are population features without an obvious geographical pattern. There is no significant correlation between latitude and longitude of the locality of the sample and the character states that are mentioned in the original description of the so-called subspecies. Moreover, in the surroundings of Trieste, the type locality of the nominate subspecies, only populations with some 'tortivum' shell features, like the thin lip, can be found (De Mattia \& Prodan 2006).

Some of the populations have a peculiar shell morphology (see for instance Fig. 28E-F); their taxonomical status should be investigated more thoroughly especially since the available molecular H3 analysis indicates that population "EZ0813" from Rasko Polje, corresponding to the specimen in Fig. 28E, stands apart from the others.

Cochlostoma (Clessiniella) waldemari (Wagner, 1897)

Figs 1, 3D, 14E, 15E, 16E, 31-33, 34 (top), 35 (top), 36 (bottom)

Pomatias (Auritus) waldemari A.J. Wagner, 1897: 616, pl. 9, 90 (“Ogulin”).

Cochlostoma waldemari - Welter-Schultes 2012: 103.

\section{Diagnosis}

Spotless shell. Moderately strong, more or less widely spaced ribs. Columellar lobe gently curved backwards. External lobe of type "II" in almost all the populations. The body spermiduct is huge and twisted instead of straight as in all the other species of Clessiniella.

\section{Type material}

\section{Syntypes}

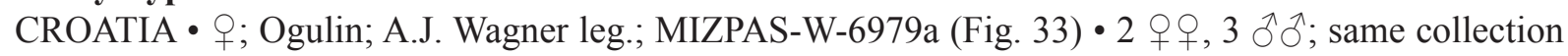
data as for preceding; MIZPAS-W-6979/5.

\section{Other material}

CROATIA - Kapela pass, Ogulin; 45.07534 N, $15.20837^{\circ}$ E; 2009; De Mattia leg.; EZ0909 • Klek Mt, close to the top; 45.25904 $\mathrm{N}, 15.14044^{\circ} \mathrm{E}$; 2009; Zallot leg.; EZ0904 • Klek Mt, cliffs halfway; $45.25438^{\circ} \mathrm{N}, 15.14561^{\circ} \mathrm{E}$; 1996; Zallot leg.; EZ0905 • Mt Risnjak; $45.43095^{\circ} \mathrm{N}, 14.6188^{\circ}$ E; 1400 m a.s.1.; 2009; De Mattia leg.; EZ0845 • Mt Risnjak; $45.39072^{\circ} \mathrm{N}, 14.61748^{\circ}$ E; $1000 \mathrm{~m}$ a.s.1.; 2010; Zallot leg.; EZ0907 • Ročko Polje; 45.3607 N, 14.09534 E; 2009; De Mattia leg.; WdM6697 - Rudnica; $45.23402^{\circ} \mathrm{N}, 15.33827^{\circ}$ E; 2009; De Mattia leg.; EZ0906 - Spilja Vrelo; $45.31777^{\circ} \mathrm{N}$, 14.71065 E; 2009; Zallot leg.; EZ0899 • Vitunj-Ogulin; $45.29114^{\circ} \mathrm{N}, 15.14041^{\circ} \mathrm{E} ; 2005$; Zallot leg.; EZ0903.

SLOVENIA •Benete, $45.76192^{\circ} \mathrm{N}, 14.56752^{\circ} \mathrm{E}$; 2009; De Mattia leg.; WdM6805 • Bresovica; $45.95607^{\circ} \mathrm{N}$, 14.43533ํ E; 2006; De Mattia leg.; EZ0897 • Bresovica pri Predgradu; $45.55164^{\circ}$ N, $15.0437^{\circ}$ E; 2009; De Mattia leg.; WdM6612 • Divje Jezero; $45.9827^{\circ} \mathrm{N}, 14.0277^{\circ}$ E; 2009; De Mattia leg.; WdM6840 • Dol-E of Čepovan; $46.06445^{\circ} \mathrm{N}, 13.81189^{\circ} \mathrm{E}$; 2009; De Mattia leg.; WdM6837 • Jelenja Vas; $45.5115^{\circ} \mathrm{N}$, 15.05346 ${ }^{\circ}$ E; 2009; De Mattia leg.; WdM6804 • Krempa Kolpa; $45.53417^{\circ}$ N, $15.09528^{\circ}$ E; 1996; Mildner leg.; EZ0902 • Marija Ceplje Kolpa; 45.53444 N, 15.09389 E; 2006; Mildner leg.; EZ0901 • Mt Snežnik 1150; 45.56913 ${ }^{\circ}$ N, $14.321^{\circ}$ E; Jun. 2010; De Mattia leg.; WdM7014 • Mt Snežnik 1620; $45.58845^{\circ}$ N, 14.44209 E; Jun. 2010; De Mattia leg.; EZ0963 • Mt Snežnik car parking; $45.58235^{\circ}$ N, $14.43117^{\circ}$ E; Jun. 2010; De Mattia leg.; WdM7006 • Polog-E of Čepovan; 46.06387 N, 13.81275 E; 2009; De Mattia leg.; WdM6842 • SW Pescena Glava; 46.05156 N, $13.73844^{\circ}$ E; 843 m a.s.1.; 2009; De Mattia leg.; WdM6829 
- Tisovec; $45.81809^{\circ} \mathrm{N}, 14.76859^{\circ} \mathrm{E}$; 2009; De Mattia leg.; WdM6749 • same collection data as for preceding; WdM6750 • Vrsic Pass; $46.44278^{\circ} \mathrm{N}, 13.76417^{\circ}$ E; 1985; Zallot leg.; EZ0896 • ZagradecPolyane; $45.86028^{\circ} \mathrm{N}, 14.83417^{\circ} \mathrm{E}$; Bodon leg.; EZ0900.

\section{Description}

SHELL. The shell is more or less light corneous brown and spotless. The size of the aperture varies considerably among the populations; shells with a large aperture and a correspondingly large lip occur next to shells with a poorly developed lip and a relatively small aperture. The lip is gently curved backwards on the columellar side.

Female genitalia. The female genitalia are hardly distinguishable from those of $\mathrm{Co}$. (Cl.) tergestinum if not for the more relaxed loops.

Male genitalia. There is a long, inflated penis. The penial spermiduct is slightly twisted and occupies only the central part of the penis. The body spermiduct is twisted and conspicuously protruding (see Figs 34-35).

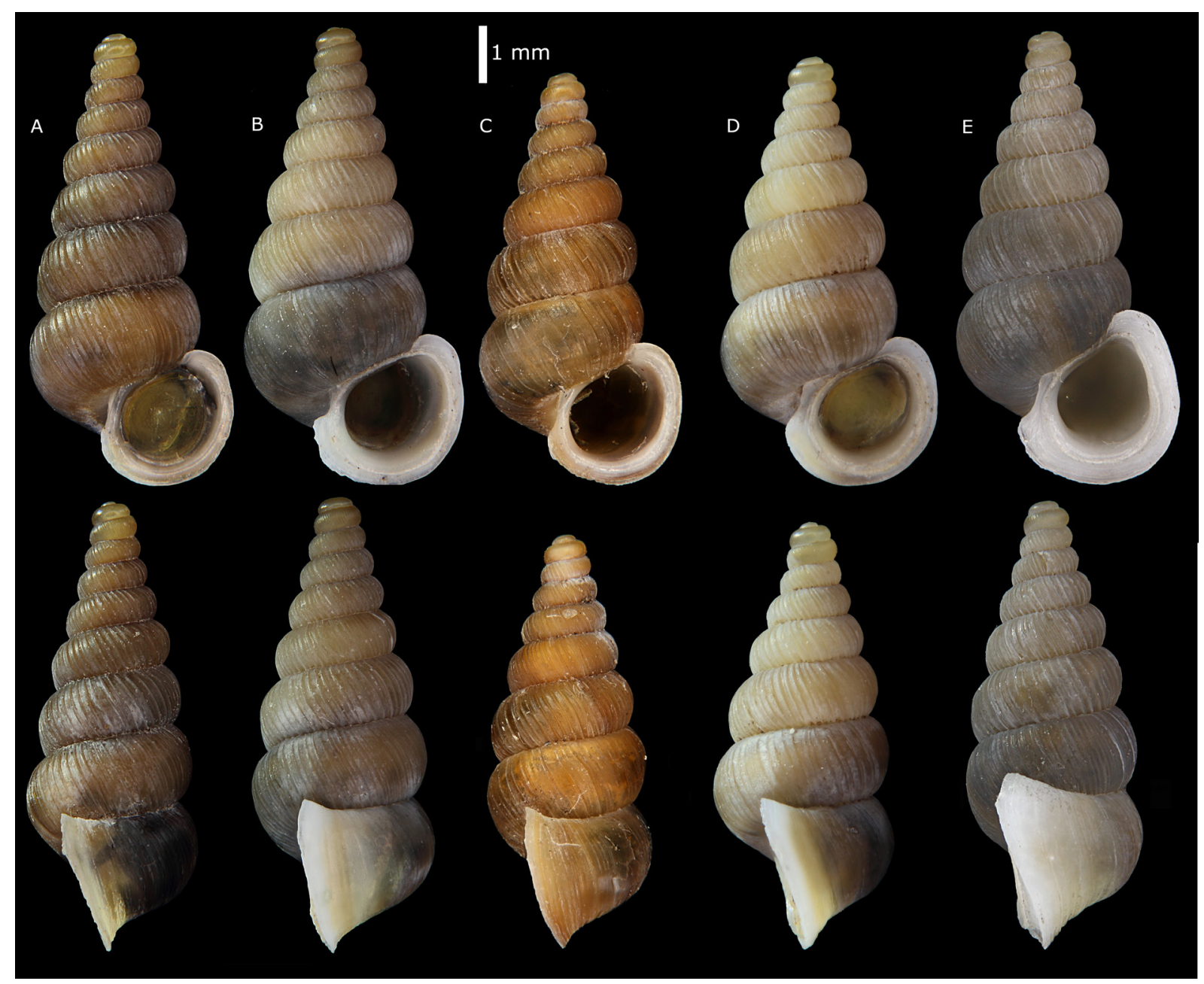

Fig. 31. Shell variability in Cochlostoma (Clessiniella) waldemari (A.J. Wagner, 1897). A. Polog-E of Čepovan, Slovenia (WdM6842). B. Vrsic Pass, Slovenia (EZ0896). C. Benete, Slovenia (WdM6805). D. Mt Klek, Croatia (EZ0904). E. Bresovica pri Predgradu, Slovenia (WdM6612). 


\section{Distribution}

The species inhabits the north-western and south-eastern part of Slovenia and an adjacent area in Croatia. It is reported from Austria for the Karawanken and the Gailtaler Alpen (Edlinger \& Mildner 1979). Its range largely surrounds that of $\mathrm{Co}$. $(\mathrm{Cl}$.) tergestinum (see Fig. 1).

\section{Habitat preference}

The snails are found on rocky cliffs as well as on boulders in woody areas.

\section{Remarks}

To assign a given population to $\mathrm{Co}$. $(\mathrm{Cl}$.) waldemari rather than to $\mathrm{Co}$. $(\mathrm{Cl}$.) tergestinum on the basis of the morphology of the shell and the female genitalia alone could be challenging if not impossible (see Figs 34-35). Only the structure of the male genitalia allows a convincing identification of the species. The $\mathrm{H} 3$ analysis (Fig. 7) confirms that these sibling species belong to separate clades within Clessiniella.

Boeckel (1939) considered Co. $(C l$.) waldemari a subspecies of $C o$. $(C l$.$) tergestinum. This view was$ contradicted by Bole (1994). In some areas, like on Mount Snežnik in southern Slovenia, the two species live not far from each other and here they can be distinguished also conchologically while differing in the presence or absence of the spots on the teleoconch (with $\mathrm{Co}$. ( $\mathrm{Cl}$.) waldemari having a spotless shell) and in the shape of the body whorl approaching the aperture, in lateral view (Fig. 36).

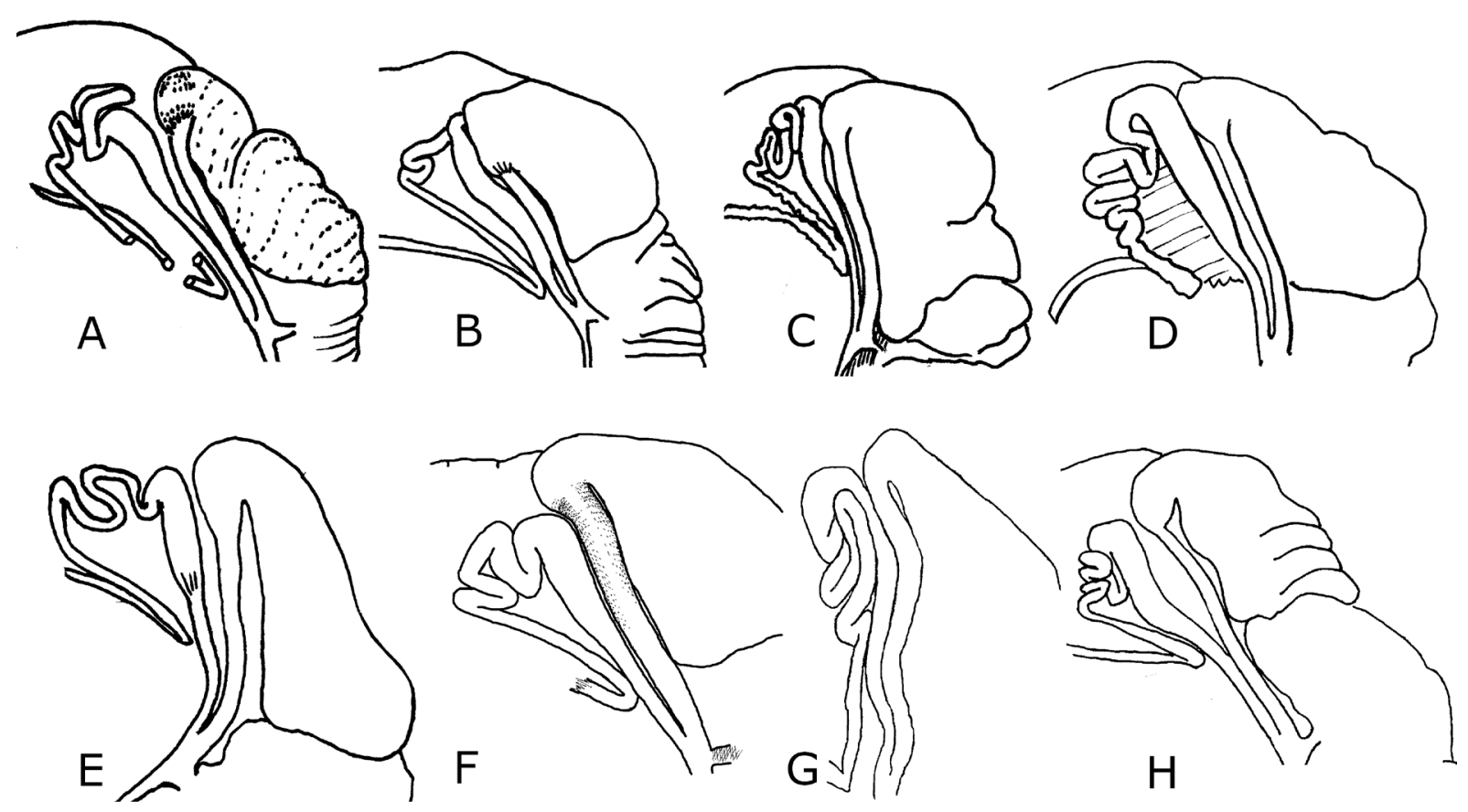

Fig. 32. Variation of the female genitalia in (A.J. Wagner, 1897). A. Polog-E of Čepovan, Slovenia (WdM6842). B. Dol-E of Čepovan, Slovenia (WdM6837). C. Divje Jezero, Slovenia (WdM6840). D. Tisovec, Slovenia (WdM6749). E. Vrsic Pass, Slovenia (EZ0896). F. Benete, Slovenia (WdM6805). G. Vitunin spring, Croatia (EZ0903). H. Mt Klek, Croatia (EZ0905). 


\section{Discussion}

The lack of clear diagnostic features in shell morphology, with intraspecific sometimes overlapping interspecific variability as in Pyrenean Cochlostomatidae (Gofas 2001), is one of the reasons of the problematic taxonomy at the species level within the genus Cochlostoma. Based on only shell morphology, several populations of 4 of the 5 species of Clessiniella cannot be identified with certainty. The lack of clear distinguishing shell features may explain why a species like $\mathrm{Co}$. (Cl.) stelucarum sp. nov. went unnoticed in an area that may be considered well explored. The multivariate analyses of

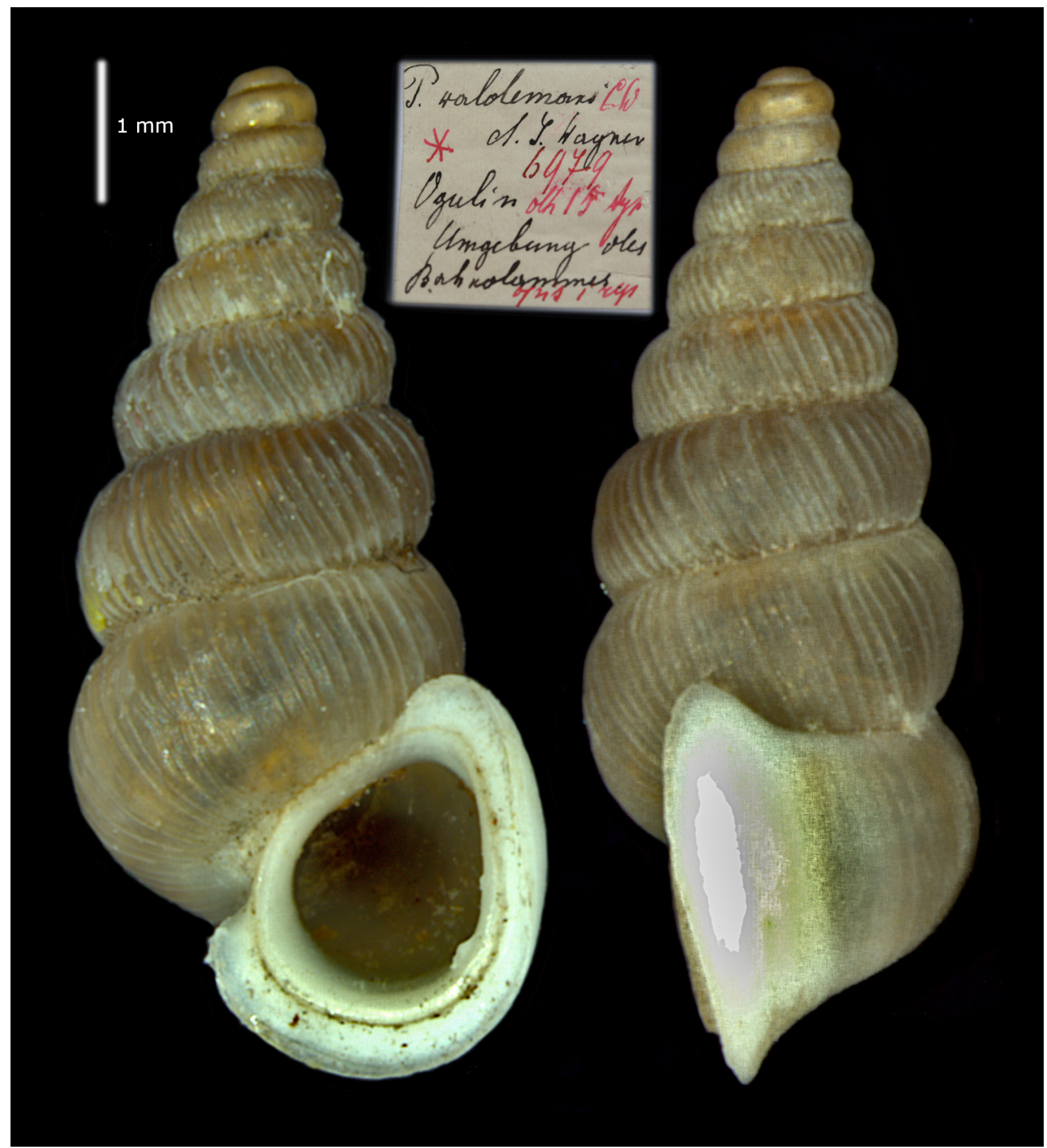

Fig. 33. Cochlostoma (Clessiniella) waldemari (A.J. Wagner, 1897), syntype, $q$, at the Muzeum i Instytut Zoologii PAN, Warsaw, Poland (MIZPAS-W-6979a). 

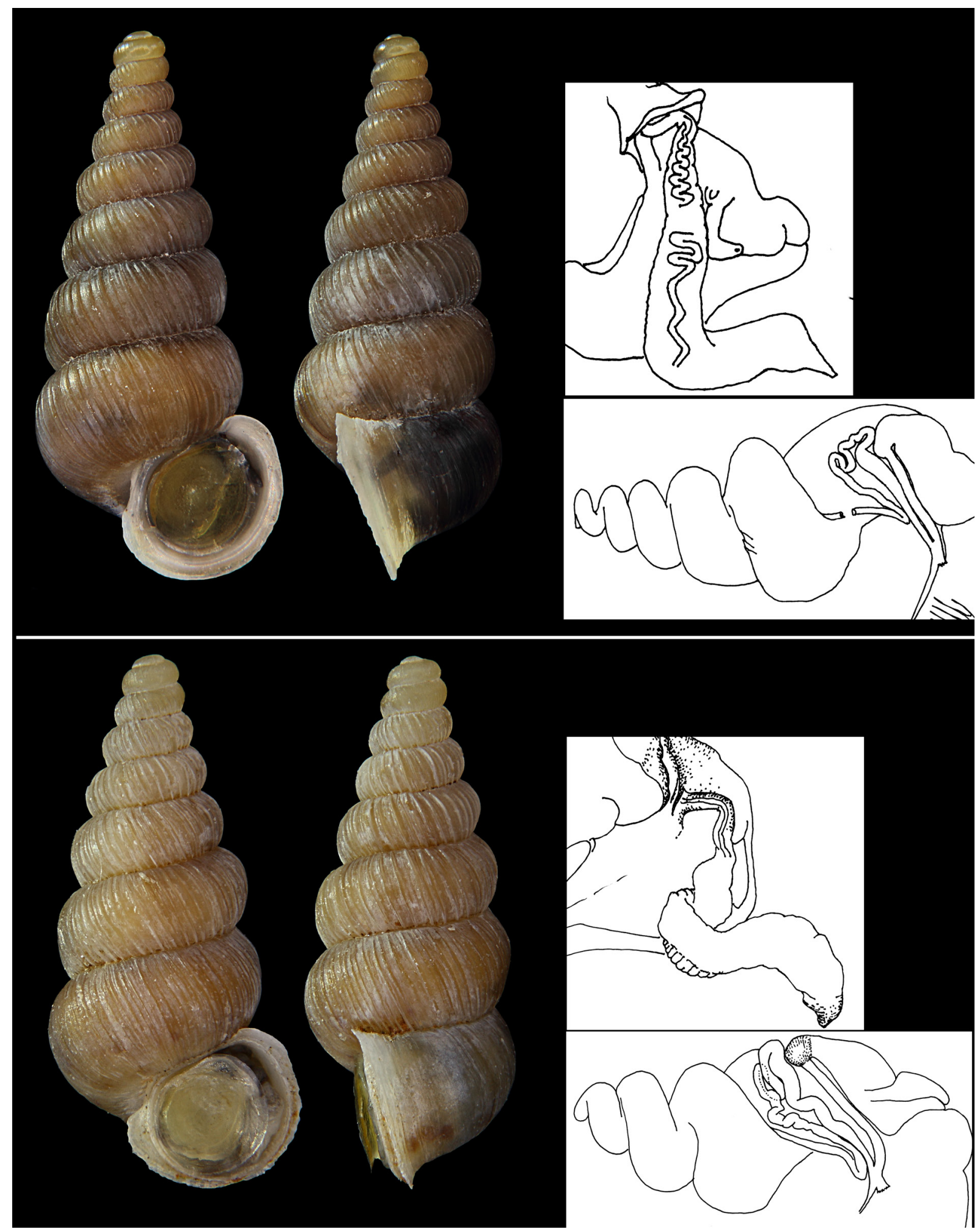

Fig. 34. Example of two populations of species of Clessiniella Zallot et al., 2015 which are impossible to distinguish without analyzing the male genitalia. Female shells. Top: Cochlostoma (Clessiniella) waldemari (A.J. Wagner, 1897), Polog-E of Čepovan, Slovenia (WdM6842). Bottom: Co. (Cl.) tergestinum (Westerlund, 1878), Jakačici, Croatia (WdM6699). 
the shell measurements also fail to unequivocally diagnose the species. The shell features that cannot be quantified may be helpful for identification, but are hardly decisive, except for the identification of $\mathrm{Co}$. $(\mathrm{Cl}$.) anomphale. There is no general, always satisfying, single morphological dataset that enables indisputable identifications. The analysis of the female genital morphology sets apart $\mathrm{Co}$. $(\mathrm{Cl}$.) anomphale and $\mathrm{Co} .(\mathrm{Cl}$.) stelucarum sp. nov. from the other species but it does not clarify everything in Cochlostoma taxonomy. Similarly, the investigation of the male genitalia reveals a taxonomical relevant feature for the diagnosis of $\mathrm{Co} .(\mathrm{Cl}$.) waldemari but not of the other species.

A combined analysis of shell and genitalia is needed to clarify the taxonomic relationships within the genus in a satisfactory way. It should not be overlooked, however, that the results of our approach do not
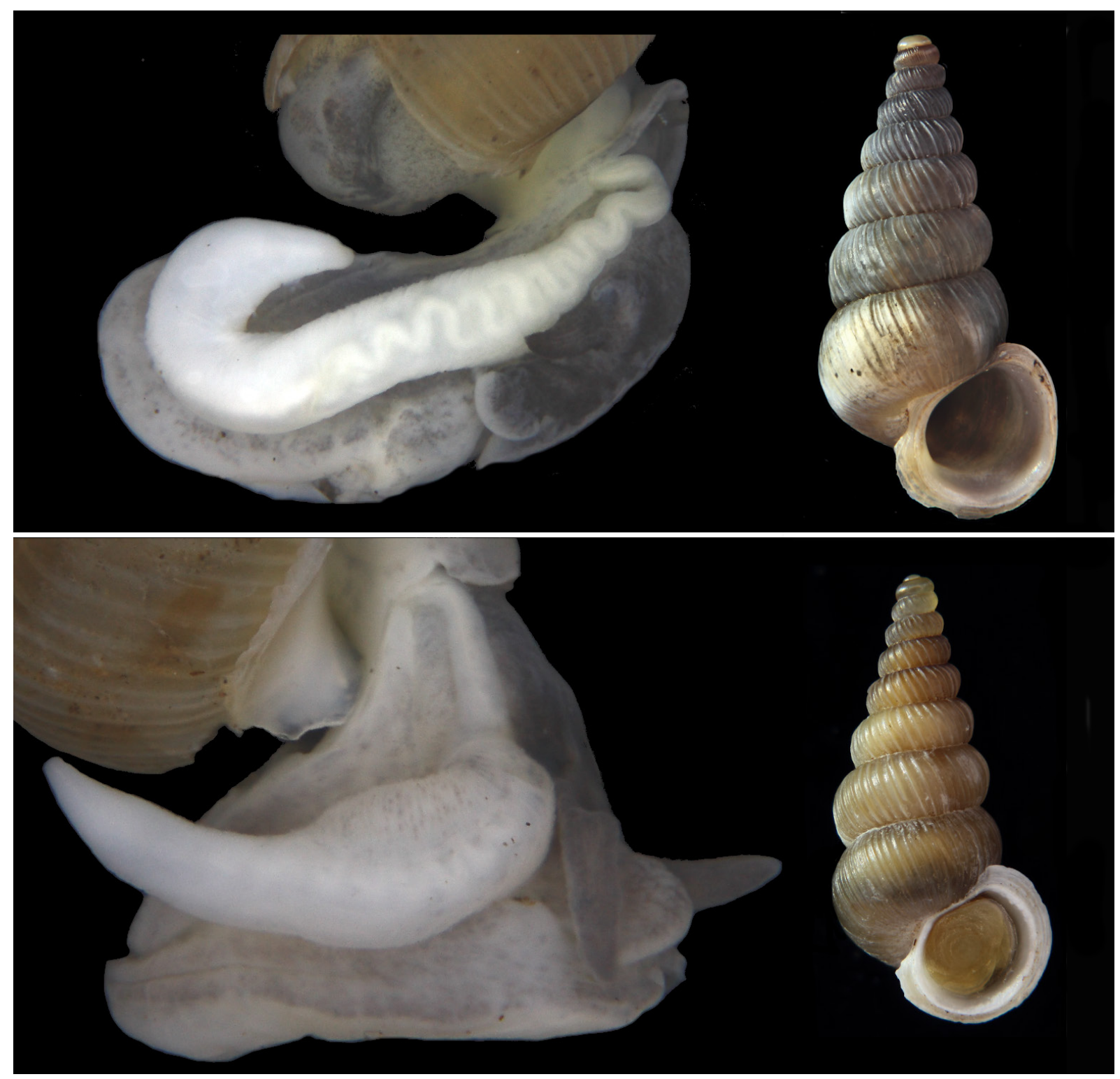

Fig. 35. Example of two populations of species of Clessiniella Zallot et al., 2015 which are impossible to distinguish without analyzing the male genitals. Male shells. Top: Cochlostoma (Clessiniella) waldemari (A.J. Wagner, 1897), Mt Klek, Croatia (EZ0904). Bottom: Co. (Cl.) tergestinum (Westerlund, 1878), Val Rosandra, Italy (EZ0840). 
fundamentally differ from the views of our predecessors except for the phylogenetic relations amongst taxa. Three species of Cochlostoma (Clessiniella) got their names in the $19^{\text {th }}$ century, one in the $20^{\text {th }}$ century, and one species was discovered only in modern times.

\section{Acknowledgements}

The authors are grateful to Dr Daniele Giannetti and Dr Cristina Menta of the Universita' degli studi di Parma (Italy) for the kind collaboration in finding and taking pictures of Strobel's material. We thank Roberta Salmaso of the Museo civico di Storia Naturale di Verona (Italy) for all the information and pictures of the De Betta material. We thank Sigrid Hof of the Senckenberg Forschungsinstitut for the photos of the types of $\mathrm{Co}$. $(\mathrm{Cl}$. $)$ anomphale and $\mathrm{Co}$. $(\mathrm{Cl}$.) tergestinum. We are grateful to Dr Fernando Scarlassara for the precious information about $\mathrm{Co}$. $(\mathrm{Cl}$. $)$ villae.
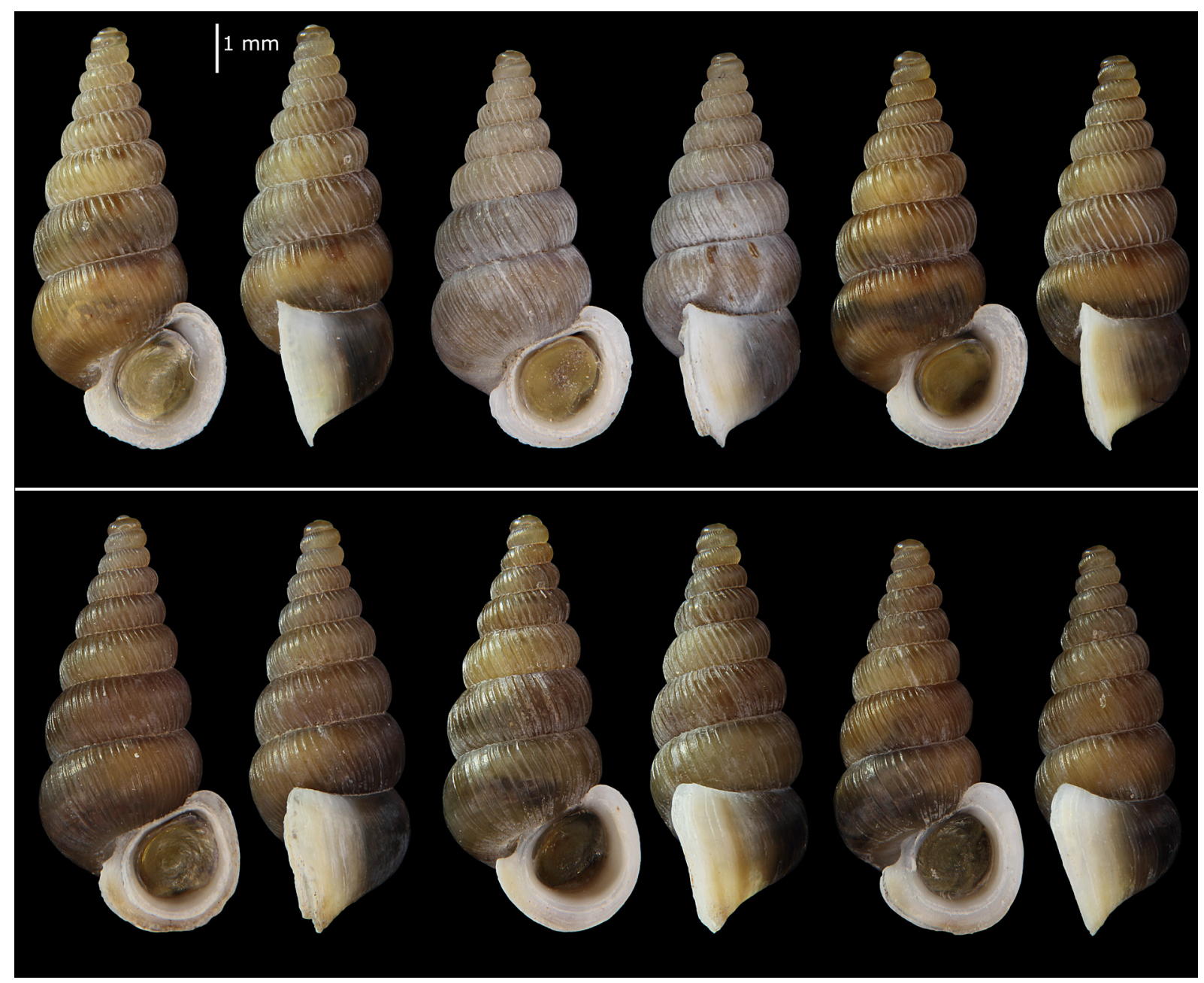

Fig. 36. Top: Cochlostoma (Clessiniella) tergestinum (Westerlund, 1878), Mt Snežnik $1161 \mathrm{~m}$ a.s.1., Slovenia (WdM7012). Bottom: Co. (Cl.) waldemari (A.J. Wagner, 1897), Mt Snežnik 1150 m a.s.l., Slovenia (WdM7014). 


\section{References}

Alzona C. 1971. Malacofauna Italica. Catalogo e bibliografia dei molluschi viventi, terrestri e d'acqua dolce. Atti della Società Italiana di Scienze Naturali e del Museo Civico di Storia Naturale di Milano 111: 1-433.

Bank R.A. 1988. Revision der nordostitalienischen Arten und Unterarten der Gattung Cochlostoma Jan, 1830 (Gastropoda Prosobranchia: Cyclophoridae). Basteria 52 (4/6): 151-170.

Boeckel W. 1939. Cochlostoma-Arten aus den Karawanken. Archiv für Molluskenkunde 71: 41-50.

Bole J. 1994. Rod Cochlostoma Jan 1830 (Gastropoda, Prosobranchia, Cochlostomatidae) v Sloveniji. Razprave [Dissertationes], Slovenska Akademija Znanosti in Umetnosti [Academia Scientiarum et Artium Slovenica], Razred za Naravoslovne Vede [Classis 4] 35 (11): 187-217.

Clement M., Snell Q., Walker P., Posada D. \& Crandall K. 2000. TCS: estimating gene genealogies. Proceedings $16^{\text {th }}$ International Parallel and Distributed Processing Symposium 2002: 7.

https://doi.org/10.1109/IPDPS.2002.1016585

Colgan D.J., Ponder W.F. \& Eggler P.E. 2000. Gastropod evolutionary rates and phylogenetic relationships assessed using partial 28S rRNA and histone H3 sequences. Zoologica Scripta 29: 29-63.

https://doi.org/10.1046/j.1463-6409.2000.00021.x

De Betta E. \& Martinati P. 1855. Catalogo dei Molluschi Terrestri e Fluviatili Viventi nelle Provincie Venete di Edoardo de Betta e Pietropaolo Martinati. G. Antonelli, Verona.

https://doi.org/10.5962/bhl.title.13113

De Mattia W. \& Prodan M. 2006. Nuovi dati sulla distribuzione di Cochlostoma (Turritus) tergestinum tortivum (Westerlund, 1885) e Cochlostoma (Cochlostoma) scalarinum scalarinum (Villa \& Villa, 1841) in Italia (Gastropoda: Prosobranchia: Cochlostomatidae). Atti del Museo Civico di Storia Naturale di Trieste 52: 205-213.

Edlinger K. \& Mildner P. 1979. Monographie der in Kärnten lebenden Prosobranchiergattung. Cochlostoma. Carinthia II 169: 281-304.

Fehér Z. \& Erõss Z. 2009. Checklist of the Albanian mollusc fauna. Schriften zur Malakozoologie 25: $22-38$.

Gittenberger E. 1971. Zur näheren Kenntnis von Cochlostoma (Turritus) anomphale Boeckel (Prosobranchia-Cyclophoridae). Basteria 35 (6): 115-118.

Giusti F. \& Selmi M.G. 1985. The seminal receptacle and sperm storage in Cochlostoma montanum (Issel) (Gastropoda: Prosobranchia). Journal of Morphology 184: 121-133.

https://doi.org/10.1002/jmor.1051840204

Gofas S. 2001. The systematics of Pyrenean and Cantabrian Cochlostoma (Gastropoda, Cyclophoroidea) revisited. Journal of Natural History 35 (9): 1277-1369. https://doi.org/10.1080/002229301750384301

Hartmann M., Grimm H. \& Scheidt U. 2018. ERFURT: the Naturkundemuseum Erfurt. In: Beck L. (ed.) Zoological Collections of Germany. Natural History Collections: 295-309. Springer, Cham. https://doi.org/10.1007/978-3-319-44321-8_26

Leigh J.W. \& Bryant D. 2015. POPART: full-feature software for haplotype network construction. Methods in Ecology and Evolution 6: 1110-1116. https://doi.org/10.1111/2041-210X.12410

MolluscaBase eds. 2021. MolluscaBase. Available from http://www.molluscabase.org [accessed 24 Apr. 2021]. https://doi.org/10.14284/448

Strobel P. 1851. Notizie Malacostatiche sul Trentino. Fusi, Pavia. Available from https://www.biodiversitylibrary.org/page/46194631 [accessed 12 Jul. 2021]. 
Uit de Weerd D.R. \& Gittenberger E. 2013. Phylogeny of the land snail family Clausiliidae (Gastropoda: Pulmonata). Molecular Phylogenetics and Evolution 67: 201-216. https://doi.org/10.1016/j.ympev.2013.01.011

Wagner A.J. 1897. Monographie der Gattung Pomatias Studer. Denkschriften der kaiserlichen Akademie der Wissenschaften, Mathematisch-Naturwissenschaftliche Classe 64: 565-632.

https://doi.org/10.5962/bhl.title.10792

Welter-Schultes F.W. 2012. European non-marine Molluscs, a Guide for Species Identification. Planet Poster Editions, Göttingen.

Westerlund C.A. 1878. Diagnosen neuer Mollusken. Nachrichtsblatt der Deutschen Malakozoologischen Gesellschaft 10: 108-109.

Westerlund C.A. 1885. Fauna der in der paläarctischen Region (Europa, Kaukasien, Sibirien, Turan, Persien, Kurdistan, Armenien, Mesopotamien, Kleinasien, Syrien, Arabien, Egypten, Tripolis, Tunesien, Algerien und Marocco) lebenden Binnenconchylien. V. Fam. Succinido, Auriculidœ, Limnoeidoe, Cyclostomidae \& Hydrocenidce. Lund.

Zallot E. 2002. Alcune note sul genere Cochlostoma Jan, 1830 (Gastropoda, Prosobranchia) in Friuli (Italia nord-orientale). Gortania 24: 93-113.

Zallot E., Groenenberg D.S.J., De Mattia W., Fehér Z. \& Gittenberger E. 2015. Genera, subgenera and species of the Cochlostomatidae (Gastropoda, Caenogastropoda, Cochlostomatidae). Basteria 78 (4-6): 63-88.

Zallot E., Fehér Z., Bamberger S. \& Gittenberger E. 2018. Cochlostoma revised: the subgenus Lovcenia Zallot et al., 2015 (Caenogastropoda, Cochlostomatidae). European Journal of Taxonomy 464: 1-25. https://doi.org/10.5852/ejt.2018.464

Zilch A. 1958. Die Typen und Typoide des Natur-Museums Senckenberg, 21: Mollusca, Cyclophoridae, Craspedopominae-Cochlostominae. Archiv für Molluskenkunde 87 (1/3): 53-76.

Manuscript received: 10 December 2020

Manuscript accepted: 10 June 2021

Published on: 6 August 2021

Topic editor: Rudy Jocqué

Section editor: Thierry Backeljau

Desk editor: Pepe Fernández

Printed versions of all papers are also deposited in the libraries of the institutes that are members of the EJT consortium: Muséum national d'histoire naturelle, Paris, France; Meise Botanic Garden, Belgium; Royal Museum for Central Africa, Tervuren, Belgium; Royal Belgian Institute of Natural Sciences, Brussels, Belgium; Natural History Museum of Denmark, Copenhagen, Denmark; Naturalis Biodiversity Center, Leiden, the Netherlands; Museo Nacional de Ciencias Naturales-CSIC, Madrid, Spain; Real Jardín Botánico de Madrid CSIC, Spain; Zoological Research Museum Alexander Koenig, Bonn, Germany; National Museum, Prague, Czech Republic. 